Supporting Information

\title{
Synthesis, antibacterial and anticancer evaluation of novel spiramycin-like conjugates containing C(5) triazole arm
}

\author{
Katarzyna Klich ${ }^{1}$, Krystian Pyta ${ }^{1}$, Marcelina M. Kubicka ${ }^{2}$, Piotr Ruszkowski ${ }^{3}$, Lech Celewicz ${ }^{1}$, \\ Marzena Gajecka ${ }^{2,4}$, Piotr Przybylski ${ }^{*}$ \\ ${ }^{a}$ Faculty of Chemistry, Adam Mickiewicz University, Umultowska 89b, 61-614, Poznan, Poland. \\ ${ }^{\mathrm{b}}$ Department of Genetics and Pharmaceutical Microbiology, University of Medical Sciences, \\ Swiecickiego 4, 60-781 Poznan, Poland \\ ${ }^{\mathrm{c}}$ Department od Pharmacology, Poznan University of Medical Sciences, Rokietnicka 5a, 60-806 \\ Poznan, Poland \\ Institute of Human Genetics, Polish Academy of Science, Strzeszynska 32, 60-479 Poznan, Poland \\ *E-mail: piotrp@amu.edu.pl; Fax:+48 618291505; Tel: +48 618291693
}

Table 1S. Average MIC values of 1-16 compounds $\left(S_{\text {MIC }}=0\right)$ expressed in $\mu \mathrm{g} / \mathrm{mL}$ and in $[\mu \mathrm{M}]$, evaluated in the $0.25-64 \mu \mathrm{g} / \mathrm{mL}$ range of concentrations, together with

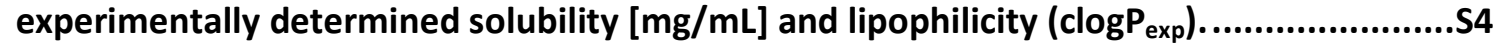

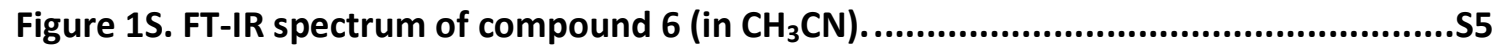

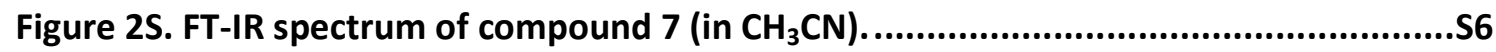

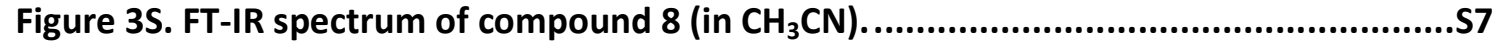

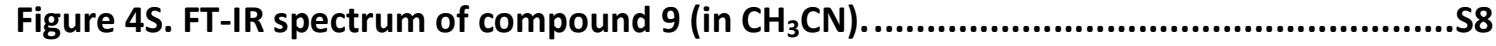

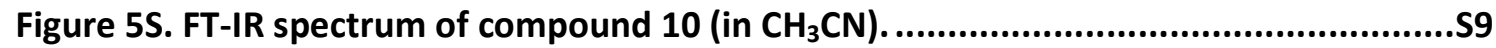

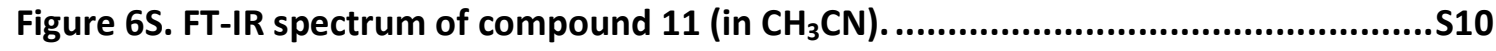

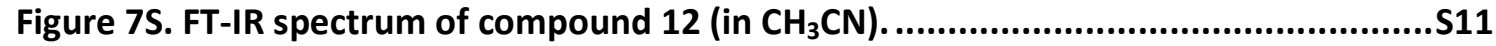

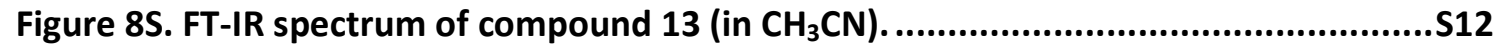

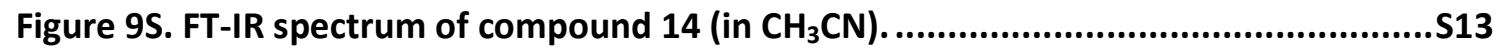

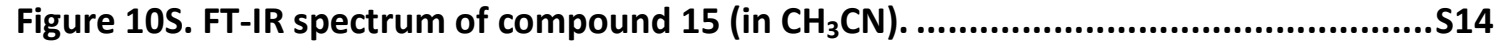

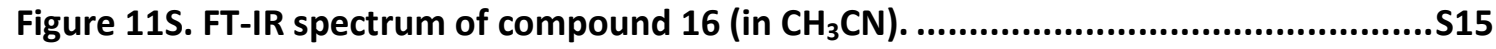

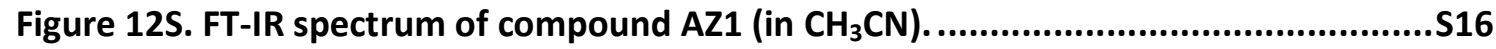

Figure 13S. FT-IR spectrum of compound AZ2 (in $\mathrm{CH}_{3} \mathrm{CN}$ ) .......................................S17

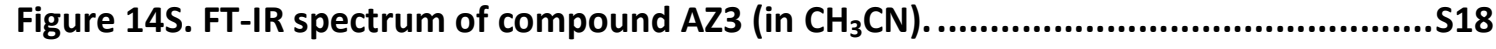

Figure 15S. FT-IR spectrum of compound AZ4 (in $\mathrm{CH}_{3} \mathrm{CN}$ ) .......................................S19 


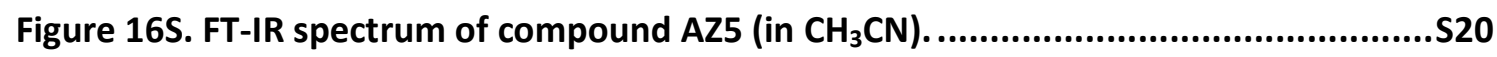

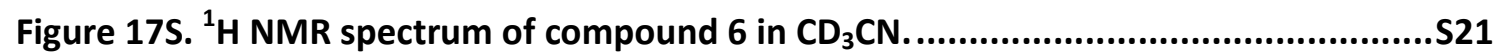

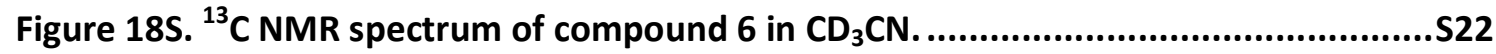

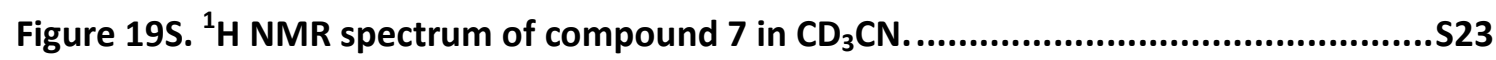

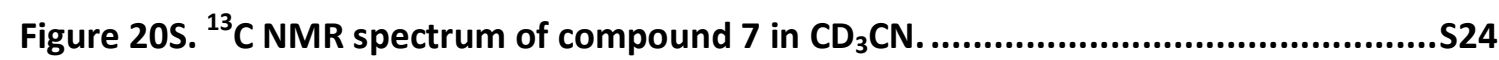

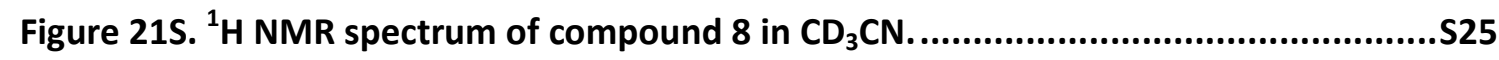

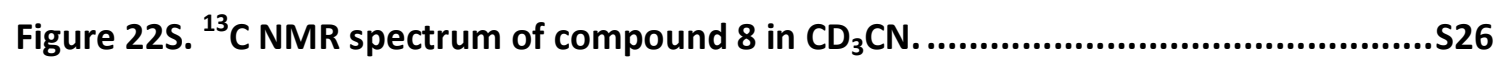

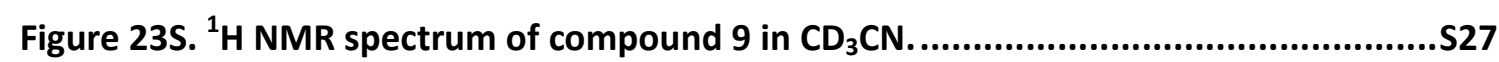

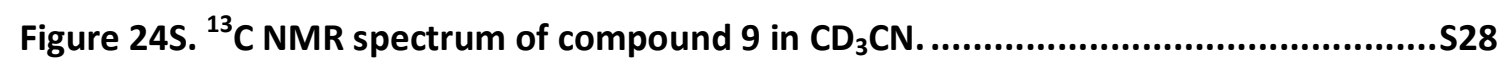

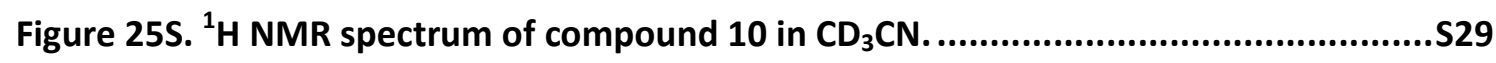

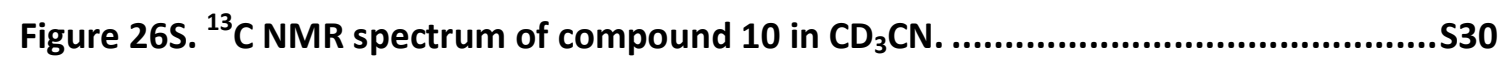

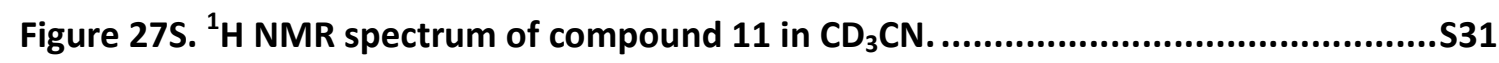

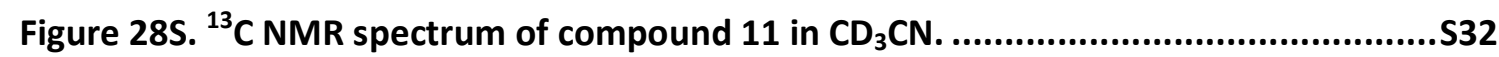

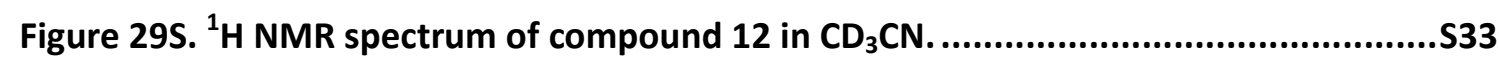

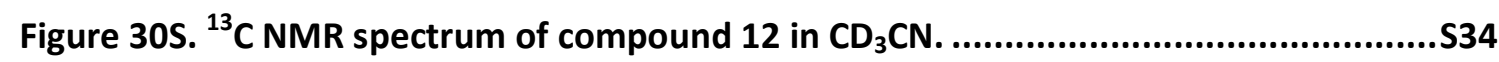

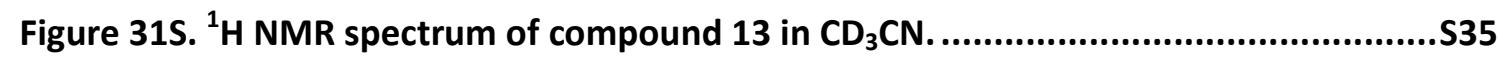

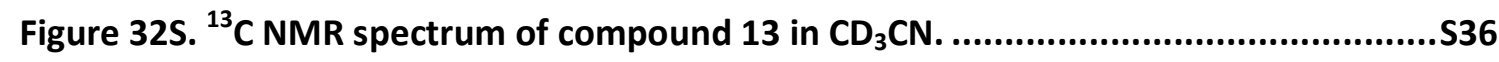

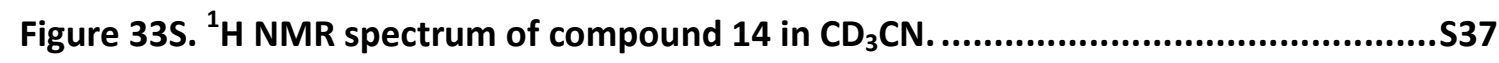

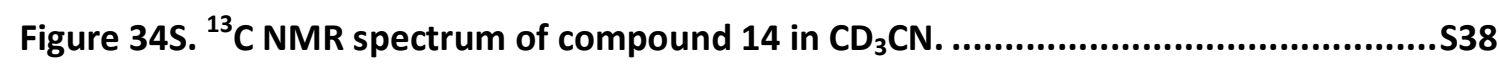

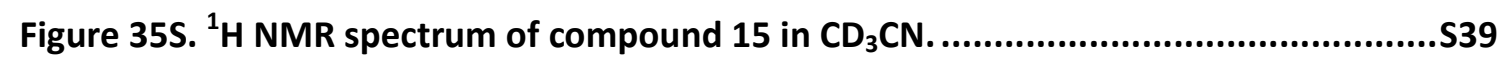

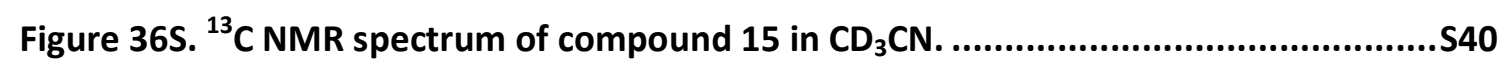

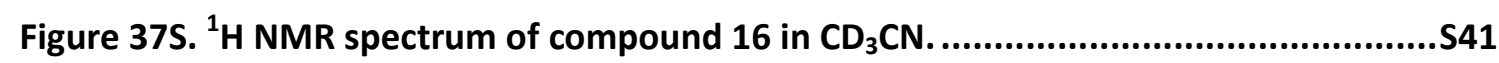

Figure 38S. ${ }^{13} \mathrm{C}$ NMR spectrum of compound 16 in $\mathrm{CD}_{3} \mathrm{CN}$........................................S42

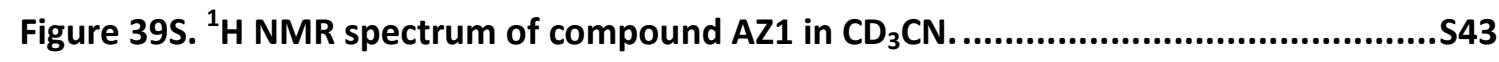

Figure 40S. ${ }^{13} \mathrm{C}$ NMR spectrum of compound $\mathrm{AZ1}$ in $\mathrm{CD}_{3} \mathrm{CN}$...................................S44

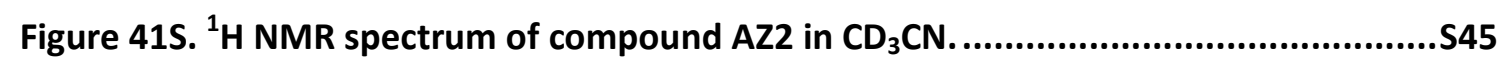

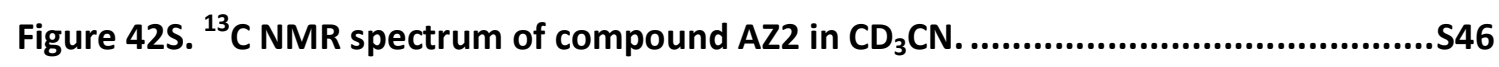

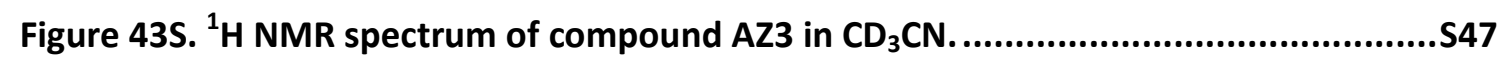

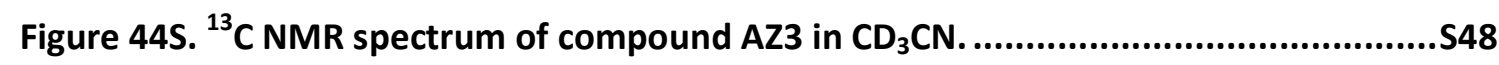

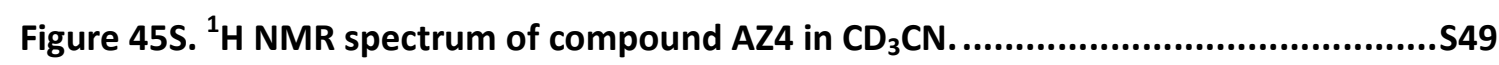

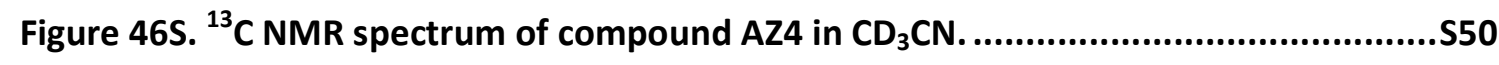

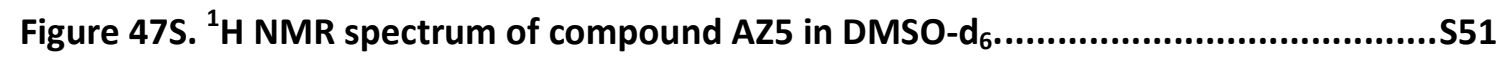




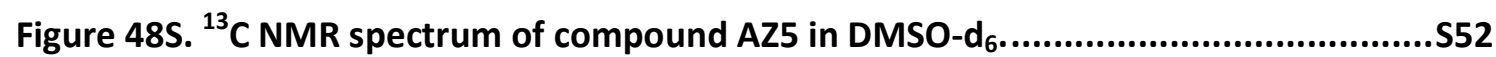

Figure 49S. Comparison of FT-IR spectra in: (a) $1850-1500 \mathrm{~cm}^{-1}$ and (b) $4000-450 \mathrm{~cm}^{-1}$ ranges between 2 (black solid) and those of novel triazole conjugates: 8 (blue shortdashed) and 11 (red long dashed); all spectra recorded in $\mathrm{CH}_{3} \mathrm{CN}$ (c $\left.0.05 \mathrm{M}\right)$. .................S53

Figure 50S. Key ${ }^{1} \mathrm{H}-{ }^{1} \mathrm{H}$ NOESY contacts recorded for compound 8 showing the mutual arrangement of the reconstructed $\mathrm{C}(5)$ arm and the aglycone part of antibiotic, shown on the lowest-energy structure $\left(\mathrm{H}_{\mathrm{f}}^{\circ}=-539.27 \mathrm{kcal} / \mathrm{mol}\right)$ calculated by B88-LYP (GGA) DFT method /Scigress Explorer package FJ 2.4. EU 3.1.8/. .554

Figure 51S. Structures of all novel chemical entities 6-16 presented in ChemDraw 8.0

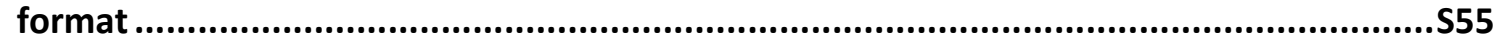


Table 1S. Average MIC values of 1-16 compounds $\left(\mathrm{SD}_{\mathrm{MIC}}=0\right)$ expressed in $\mu \mathrm{g} / \mathrm{mL}$ and in $[\mu \mathrm{M}]$, evaluated in the $0.25-64 \mu \mathrm{g} / \mathrm{mL}$ range of concentrations, together with experimentally determined solubility $[\mathrm{mg} / \mathrm{mL}]$ and lipophilicity $\left(\operatorname{clog} \mathrm{P}_{\text {exp }}\right)$.

\begin{tabular}{|c|c|c|c|c|c|c|c|c|c|}
\hline & $\begin{array}{c}\text { Bacillus } \\
\text { subtilis } \\
\text { ATCC6633 }\end{array}$ & $\begin{array}{c}\text { Micrococcus } \\
\text { luteus } \\
\text { ATCC } 10240\end{array}$ & $\begin{array}{c}\text { Staphylococcus } \\
\text { aureus } \\
\text { MRSAI }\end{array}$ & $\begin{array}{c}\text { Staphylococcus } \\
\text { aureus } \\
\text { MRSAII }\end{array}$ & $\begin{array}{c}\text { Staphylococcus } \\
\text { epidermidis } \\
\text { ATCC12228 }\end{array}$ & $\begin{array}{c}\text { Staphylococcus } \\
\text { epidermidis } \\
\text { ATCC49134 }\end{array}$ & $\begin{array}{l}\text { Streptococcus } \\
\text { pneumoniae } \\
\text { ATCC49619 }\end{array}$ & $\operatorname{clog} \mathrm{P}_{\exp }$ & $\begin{array}{c}\text { solubility }_{\exp } \\
{[\mathrm{mg} / \mathrm{mL}]}\end{array}$ \\
\hline 1 & $\begin{array}{c}0.5 \\
{[0.59]}\end{array}$ & $\begin{array}{c}0.25 \\
{[0.30]}\end{array}$ & $\begin{array}{c}8 \\
{[9.49]}\end{array}$ & $\begin{array}{c}4 \\
{[4.74]}\end{array}$ & $\begin{array}{c}2 \\
{[2.37]}\end{array}$ & $\begin{array}{c}2 \\
{[2.37]}\end{array}$ & $\begin{array}{c}0.25 \\
{[0.30]}\end{array}$ & 0.58 & 4.5 \\
\hline 2 & $\begin{array}{c}8 \\
{[16.34]}\end{array}$ & $\begin{array}{c}4 \\
{[8.17]}\end{array}$ & $\begin{array}{c}16 \\
{[32.68]}\end{array}$ & $\begin{array}{c}16 \\
{[32.68]}\end{array}$ & $\begin{array}{c}8 \\
{[16.34]}\end{array}$ & $\begin{array}{c}8 \\
{[16.34]}\end{array}$ & $\begin{array}{c}2 \\
{[4.08]}\end{array}$ & 0.74 & 0.7 \\
\hline 3 & $\begin{array}{c}>16 \\
{[>29.21]}\end{array}$ & $\begin{array}{c}>16 \\
{[>29.21]}\end{array}$ & $\begin{array}{c}>16 \\
{[>29.21]}\end{array}$ & --- & $\begin{array}{c}>16 \\
{[>29.21]}\end{array}$ & -- & $\begin{array}{c}>16 \\
{[>29.21]}\end{array}$ & 1.05 & $<0.15$ \\
\hline 4 & $\begin{array}{c}>16 \\
{[>29.32]}\end{array}$ & $\begin{array}{c}>16 \\
{[>29.32]}\end{array}$ & $\begin{array}{c}>16 \\
{[>29.32]}\end{array}$ & --- & $\begin{array}{c}>16 \\
{[>29.32]}\end{array}$ & --- & $\begin{array}{c}>16 \\
{[>29.32]}\end{array}$ & 0.85 & $<0.15$ \\
\hline 5 & $\begin{array}{c}>64 \\
{[>103.93]}\end{array}$ & $\begin{array}{c}64 \\
{[103.93]}\end{array}$ & $\begin{array}{c}>64 \\
{[>103.93]}\end{array}$ & $\begin{array}{c}>64 \\
{[>103.93]}\end{array}$ & $\begin{array}{c}>64 \\
{[>103.93]}\end{array}$ & $\begin{array}{c}>64 \\
{[>103.93]}\end{array}$ & $\begin{array}{c}>64 \\
{[>103.93]}\end{array}$ & 1.08 & $<0.15$ \\
\hline 6 & $\begin{array}{c}64 \\
{[85.23]}\end{array}$ & $\begin{array}{c}64 \\
{[85.23]}\end{array}$ & $\begin{array}{c}>64 \\
{[>85.23]}\end{array}$ & $\begin{array}{c}>64 \\
{[>85.23]}\end{array}$ & $\begin{array}{c}>64 \\
{[>85.23]}\end{array}$ & $\begin{array}{c}>64 \\
{[>85.23]}\end{array}$ & $\begin{array}{c}8 \\
{[10.65]}\end{array}$ & -0.05 & $>11$ \\
\hline 7 & $\begin{array}{c}64 \\
{[85.23]}\end{array}$ & $\begin{array}{c}64 \\
{[85.23]}\end{array}$ & $\begin{array}{c}>64 \\
{[>85.23]}\end{array}$ & $\begin{array}{c}>64 \\
{[>85.23]}\end{array}$ & $\begin{array}{c}64 \\
{[85.23]}\end{array}$ & $\begin{array}{c}64 \\
{[85.23]}\end{array}$ & $\begin{array}{c}8 \\
{[10.65]}\end{array}$ & -0.08 & $>11$ \\
\hline 8 & $\begin{array}{c}4 \\
{[5.05]}\end{array}$ & $\begin{array}{c}4 \\
{[5.05]}\end{array}$ & $\begin{array}{c}16 \\
{[20.20]}\end{array}$ & $\begin{array}{c}16 \\
{[20.20]}\end{array}$ & $\begin{array}{c}8 \\
{[10.10]}\end{array}$ & $\begin{array}{c}8 \\
{[10.10]}\end{array}$ & $\begin{array}{c}1 \\
{[1.26]}\end{array}$ & 0.25 & $>11$ \\
\hline 9 & $\begin{array}{c}>64 \\
{[>85.23]}\end{array}$ & $\begin{array}{c}>64 \\
{[>85.23]}\end{array}$ & $\begin{array}{c}>64 \\
{[>85.23]}\end{array}$ & $\begin{array}{c}>64 \\
{[>85.23]}\end{array}$ & $\begin{array}{c}>64 \\
{[>85.23]}\end{array}$ & $\begin{array}{c}>64 \\
{[>85.23]}\end{array}$ & $\begin{array}{c}>64 \\
{[>85.23]}\end{array}$ & -0.03 & $>11$ \\
\hline 10 & $\begin{array}{c}>64 \\
{[>85.23]}\end{array}$ & $\begin{array}{c}>64 \\
{[>85.23]}\end{array}$ & $\begin{array}{c}>64 \\
{[>85.23]}\end{array}$ & $\begin{array}{c}>64 \\
{[>85.23]}\end{array}$ & $\begin{array}{c}>64 \\
{[>85.23]}\end{array}$ & $\begin{array}{c}>64 \\
{[>85.23]}\end{array}$ & $\begin{array}{c}>64 \\
{[>85.23]}\end{array}$ & -0.07 & $>11$ \\
\hline 11 & $\begin{array}{c}>64 \\
{[>93.44]}\end{array}$ & $\begin{array}{c}>64 \\
{[>93.44]}\end{array}$ & $\begin{array}{c}64 \\
{[93.44]}\end{array}$ & $\begin{array}{c}64 \\
{[93.44]}\end{array}$ & $\begin{array}{c}64 \\
{[93.44]}\end{array}$ & $\begin{array}{c}64 \\
{[93.44]}\end{array}$ & $\begin{array}{c}64 \\
{[93.44]}\end{array}$ & 1.36 & 0.13 \\
\hline 12 & $\begin{array}{c}>64 \\
{[>95.40]}\end{array}$ & $\begin{array}{c}64 \\
{[95.40]}\end{array}$ & $\begin{array}{c}>64 \\
{[>95.40]}\end{array}$ & $\begin{array}{c}64 \\
{[95.40]}\end{array}$ & $\begin{array}{c}64 \\
{[95.40]}\end{array}$ & $\begin{array}{c}>64 \\
{[>95.40]}\end{array}$ & $\begin{array}{c}64 \\
{[95.40]}\end{array}$ & 1.32 & 0.13 \\
\hline 13 & $\begin{array}{c}32 \\
{[46.72]}\end{array}$ & $\begin{array}{c}32 \\
{[46.72]}\end{array}$ & $\begin{array}{c}64 \\
{[93.44]}\end{array}$ & $\begin{array}{c}64 \\
{[93.44]}\end{array}$ & $\begin{array}{c}64 \\
{[93.44]}\end{array}$ & $\begin{array}{c}64 \\
{[93.44]}\end{array}$ & $\begin{array}{c}32 \\
{[46.72]}\end{array}$ & 1.4 & 0.13 \\
\hline 14 & $\begin{array}{c}>64 \\
{[>93.17]}\end{array}$ & $\begin{array}{c}>64 \\
{[>93.17]}\end{array}$ & $\begin{array}{c}>64 \\
{[>93.17]}\end{array}$ & $\begin{array}{c}>64 \\
{[>93.17]}\end{array}$ & $\begin{array}{c}>64 \\
{[>93.17]}\end{array}$ & $\begin{array}{c}>64 \\
{[>93.17]}\end{array}$ & $\begin{array}{c}>64 \\
{[>93.17]}\end{array}$ & 1.25 & $<0.15$ \\
\hline 15 & $\begin{array}{c}>64 \\
{[>94.28]}\end{array}$ & $\begin{array}{c}>64 \\
{[>94.28]}\end{array}$ & $\begin{array}{c}>64 \\
{[>94.28]}\end{array}$ & $\begin{array}{c}>64 \\
{[>94.28]}\end{array}$ & $\begin{array}{c}>64 \\
{[>94.28]}\end{array}$ & $\begin{array}{c}>64 \\
{[>94.28]}\end{array}$ & $\begin{array}{c}64 \\
{[94.28]}\end{array}$ & 0.90 & 0.3 \\
\hline 16 & $\begin{array}{c}>64 \\
{[78.73]}\end{array}$ & $\begin{array}{c}>64 \\
{[78.73]}\end{array}$ & $\begin{array}{c}>64 \\
{[78.73]}\end{array}$ & $\begin{array}{c}>64 \\
{[78.73]}\end{array}$ & $\begin{array}{c}>64 \\
{[78.73]}\end{array}$ & $\begin{array}{c}>64 \\
{[78.73]}\end{array}$ & $\begin{array}{c}>64 \\
{[78.73]}\end{array}$ & 0.64 & 0.9 \\
\hline
\end{tabular}




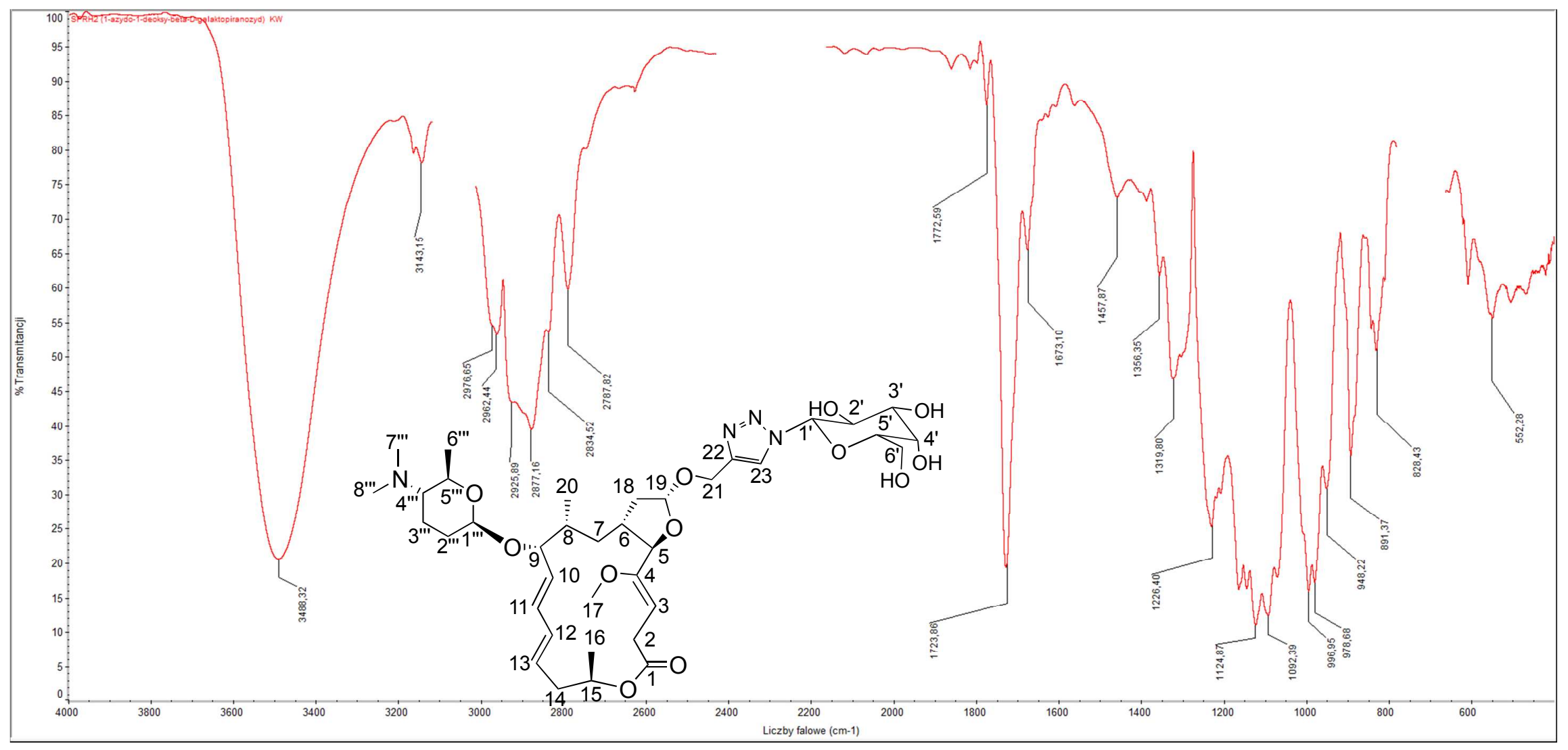

Figure 1S. FT-IR spectrum of compound 6 (in $\mathrm{CH}_{3} \mathrm{CN}$ ). 


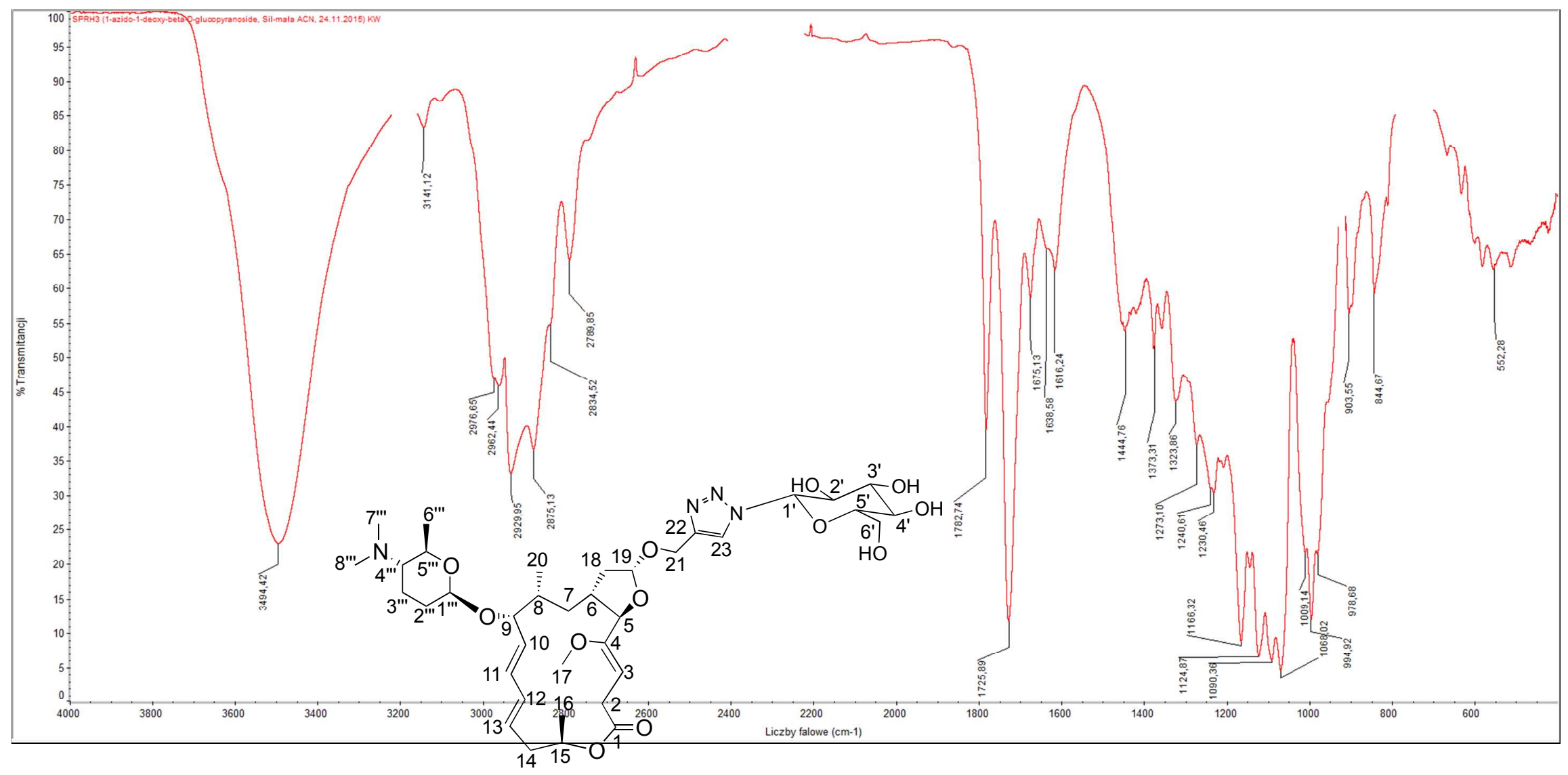

Figure 2S. FT-IR spectrum of compound 7 (in $\mathrm{CH}_{3} \mathrm{CN}$ ). 


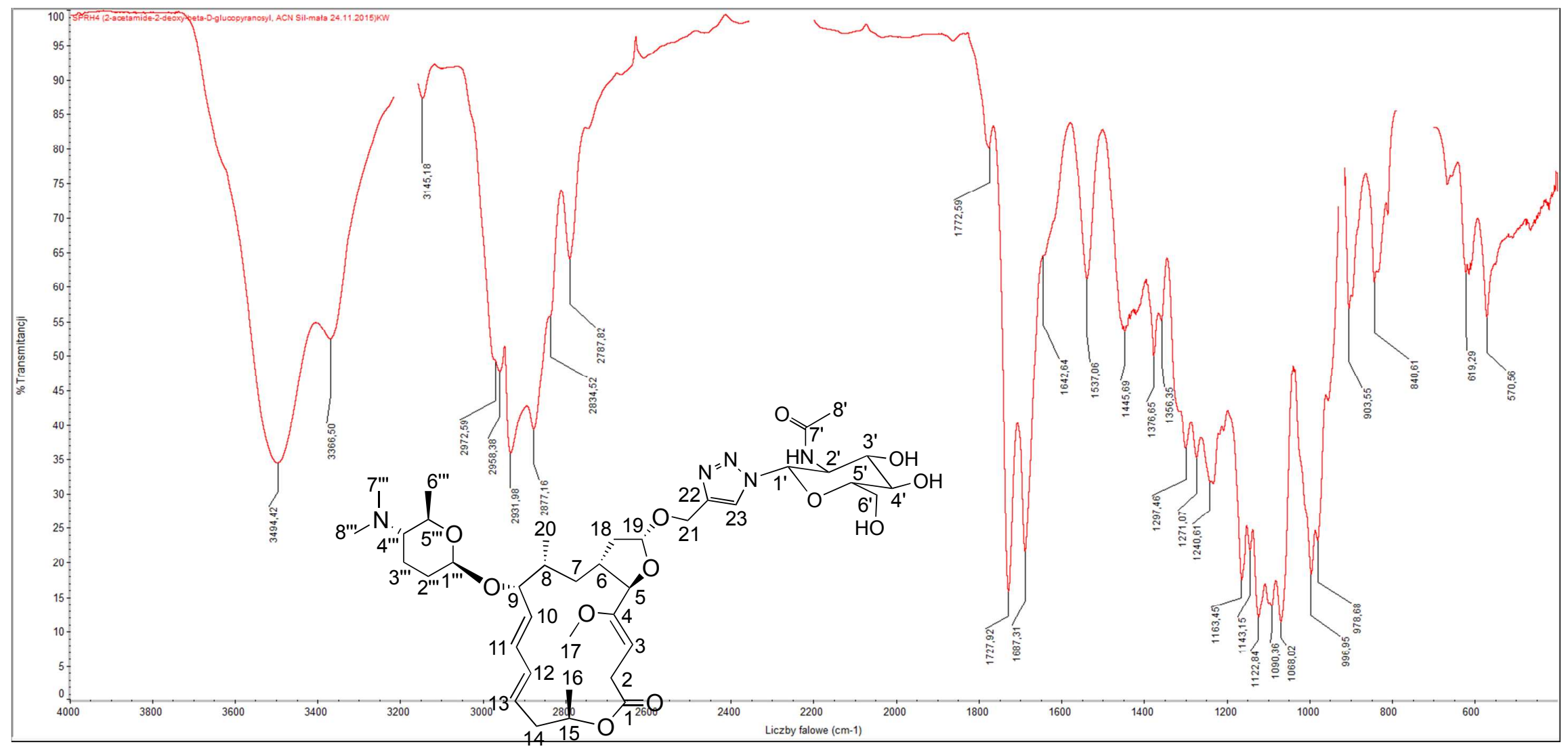

Figure 3S. FT-IR spectrum of compound 8 (in $\mathrm{CH}_{3} \mathrm{CN}$ ). 


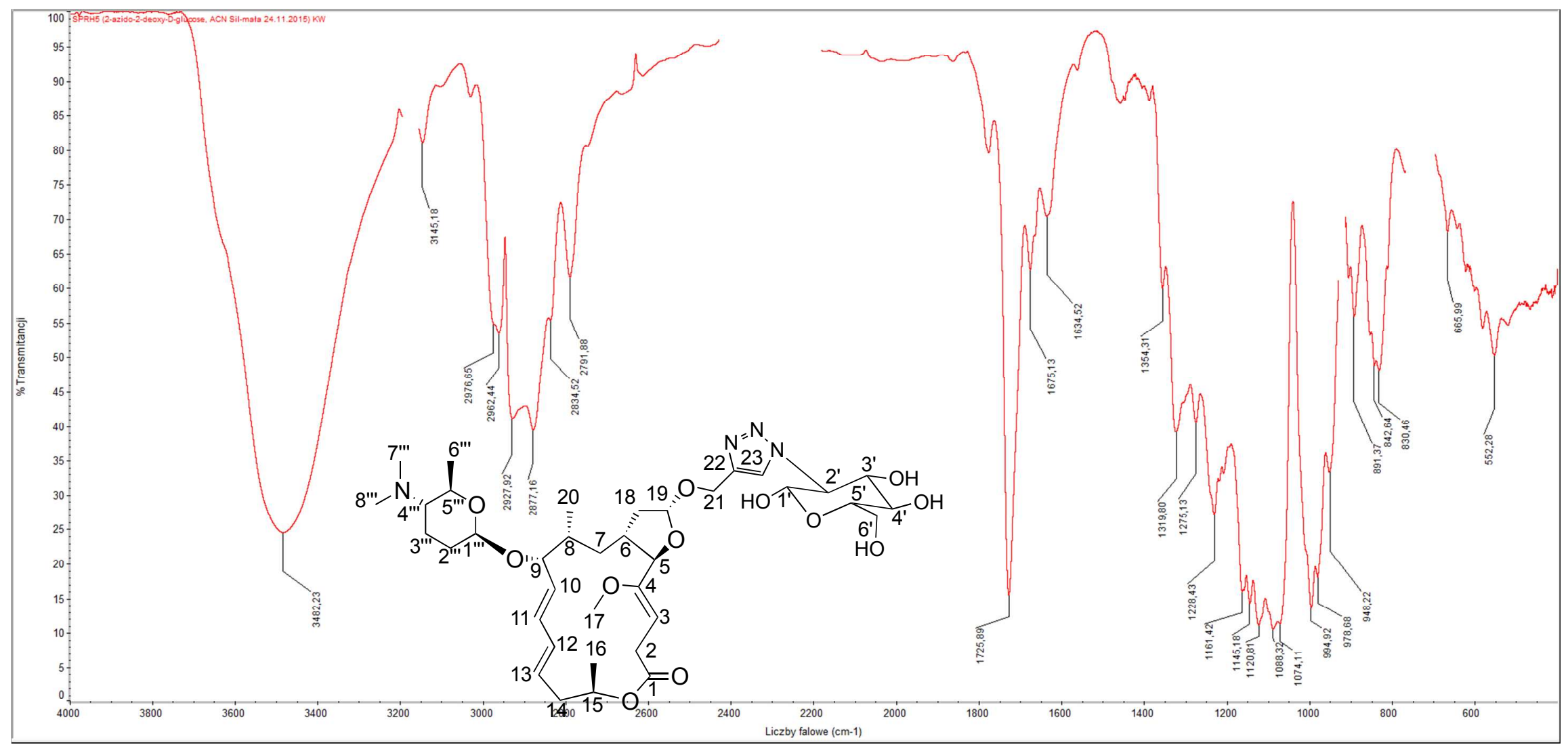

Figure 4S. FT-IR spectrum of compound 9 (in $\mathrm{CH}_{3} \mathrm{CN}$ ). 


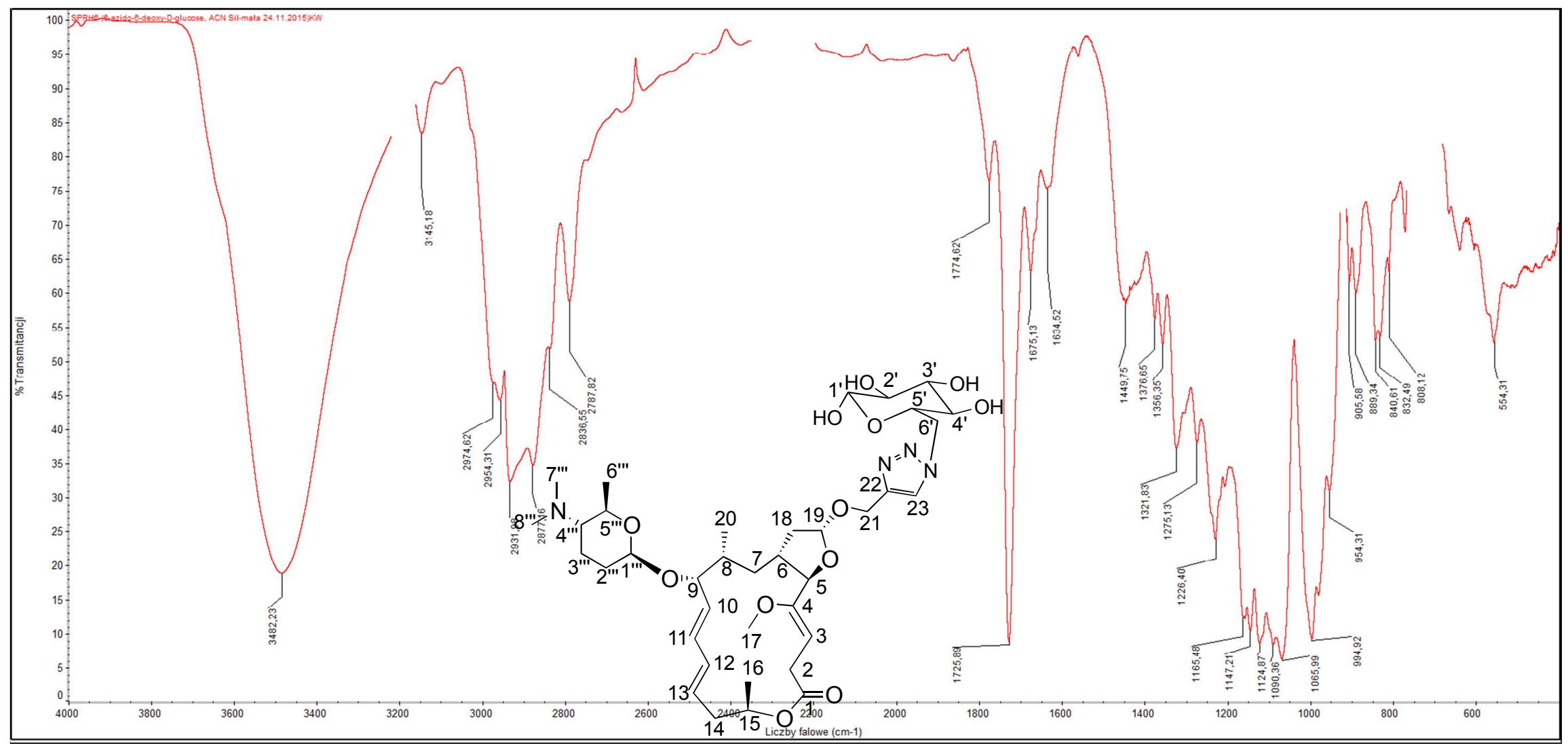

Figure 5S. FT-IR spectrum of compound $\mathbf{1 0}$ (in $\mathrm{CH}_{3} \mathrm{CN}$ ). 


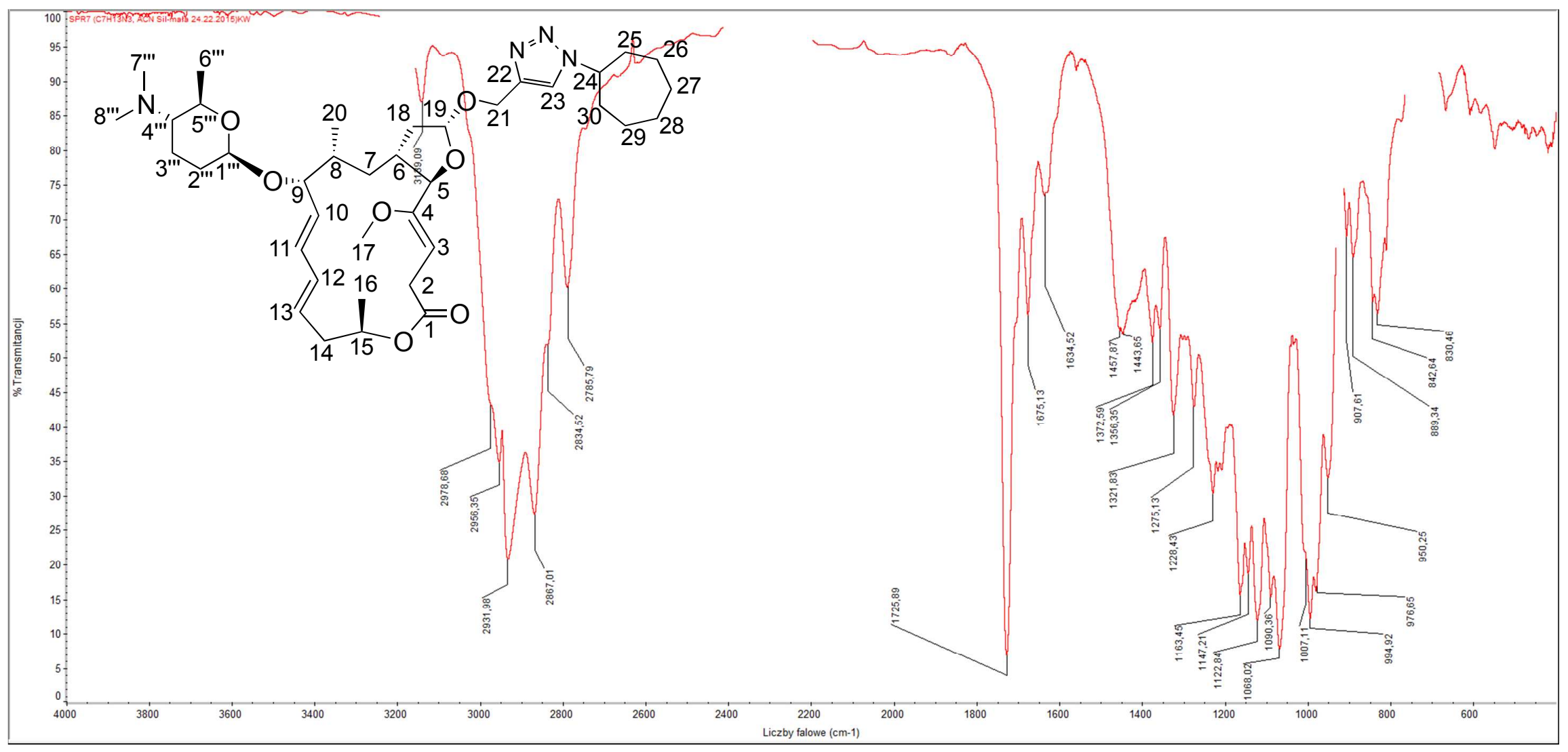

Figure 6S. FT-IR spectrum of compound $\mathbf{1 1}$ (in $\mathrm{CH}_{3} \mathrm{CN}$ ). 


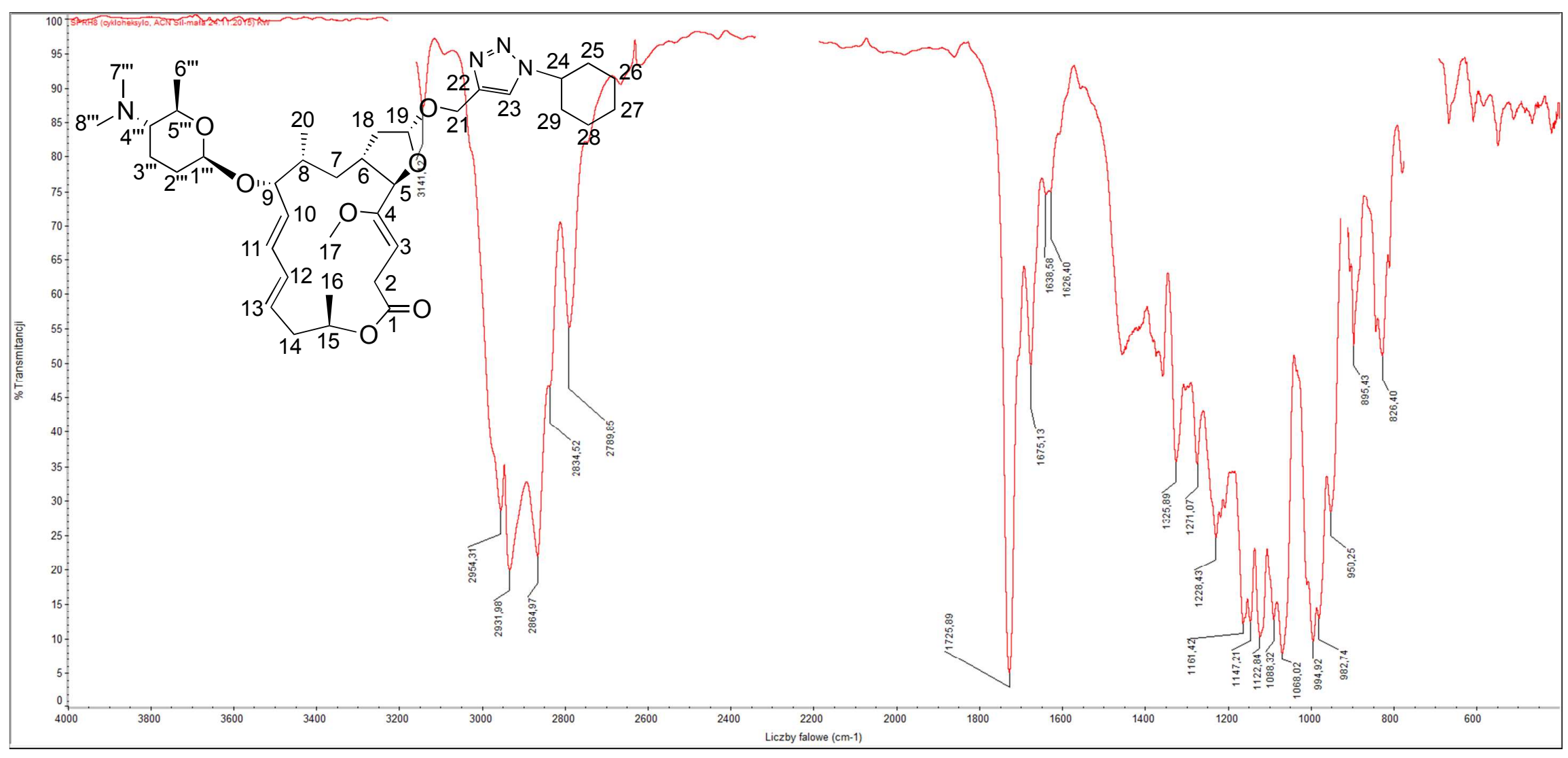

Figure 7S. FT-IR spectrum of compound 12 (in $\mathrm{CH}_{3} \mathrm{CN}$ ). 


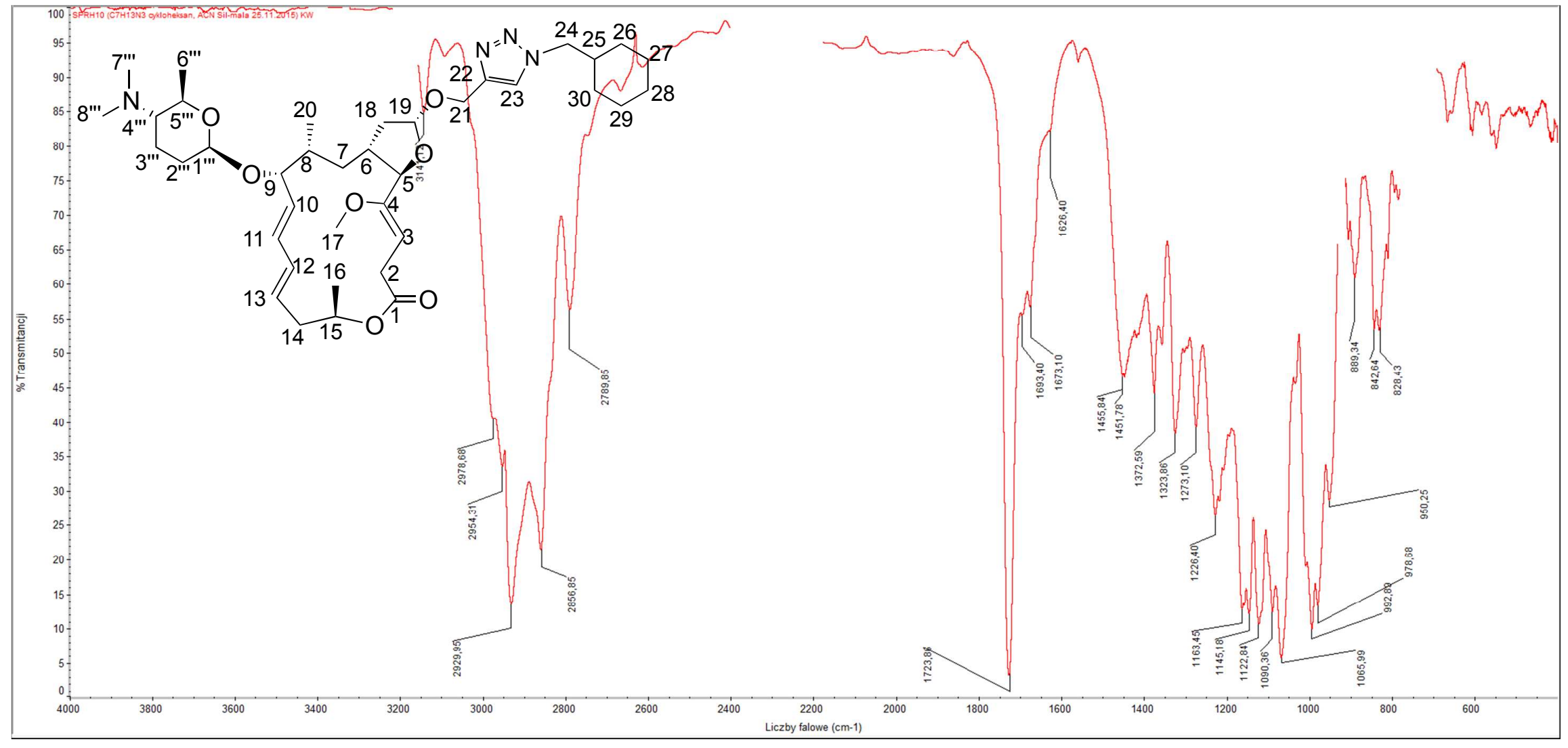

Figure 8S. FT-IR spectrum of compound $\mathbf{1 3}$ (in $\mathrm{CH}_{3} \mathrm{CN}$ ). 


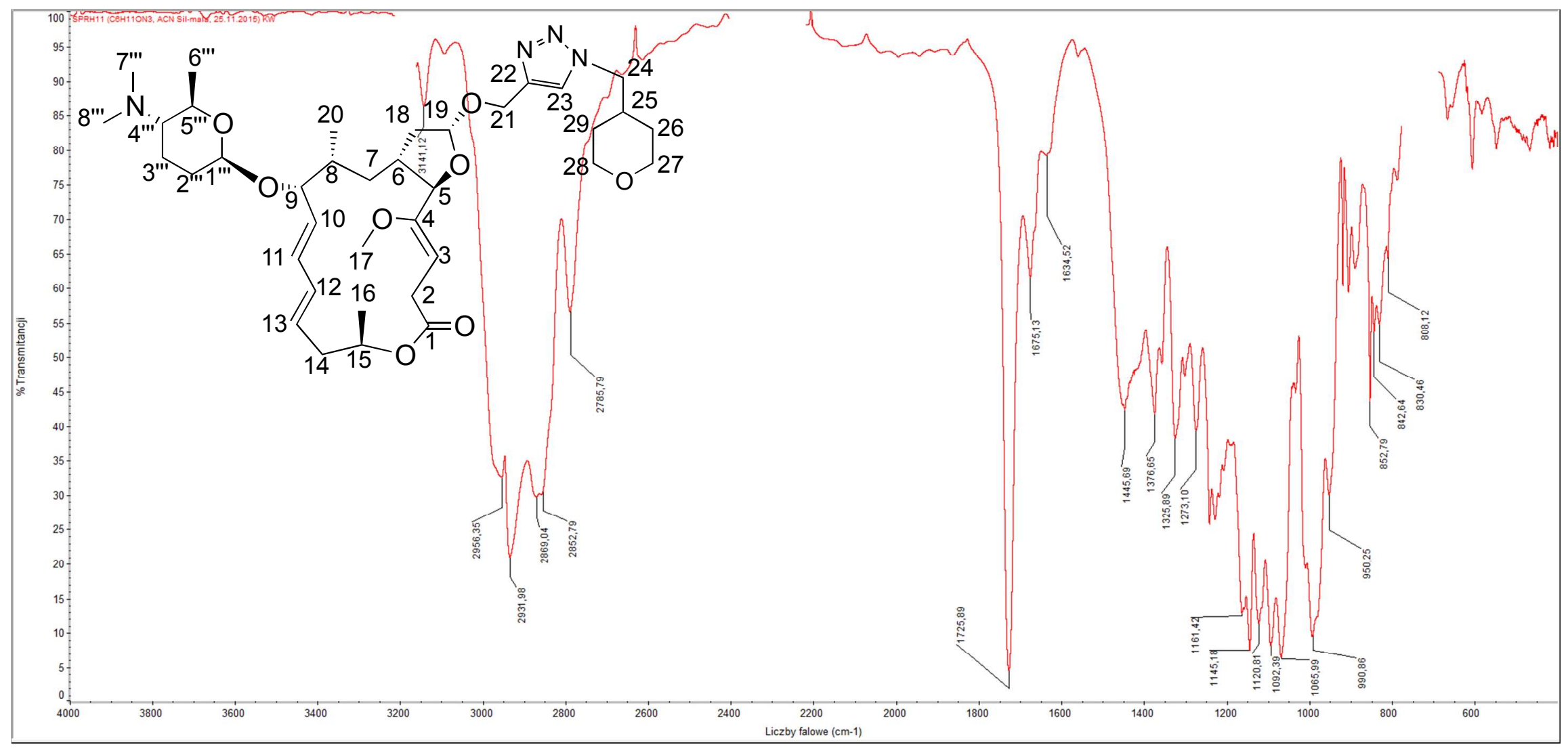

Figure 9S. FT-IR spectrum of compound $\mathbf{1 4}$ (in $\mathrm{CH}_{3} \mathrm{CN}$ ). 


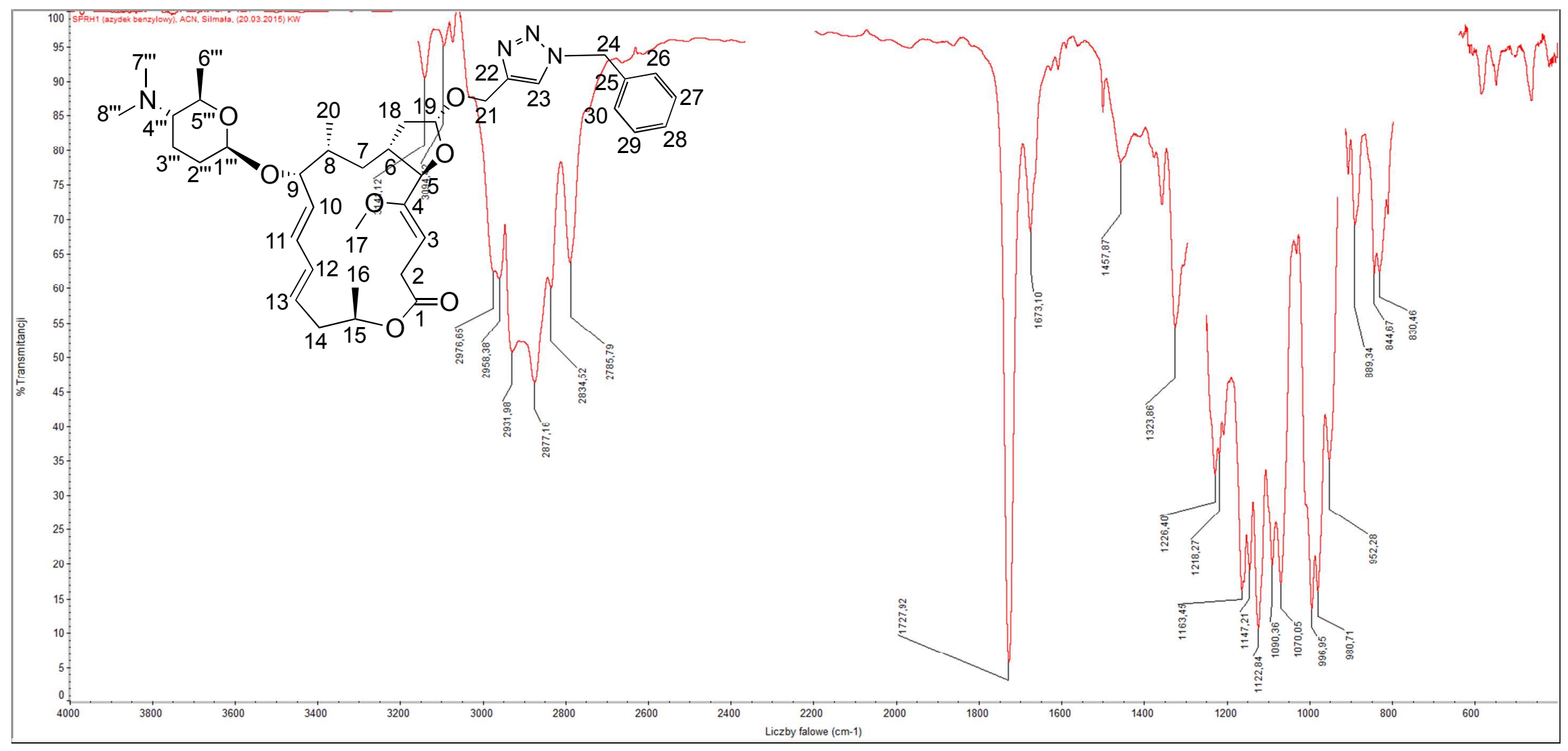

Figure 10S. FT-IR spectrum of compound $\mathbf{1 5}$ (in $\mathrm{CH}_{3} \mathrm{CN}$ ). 


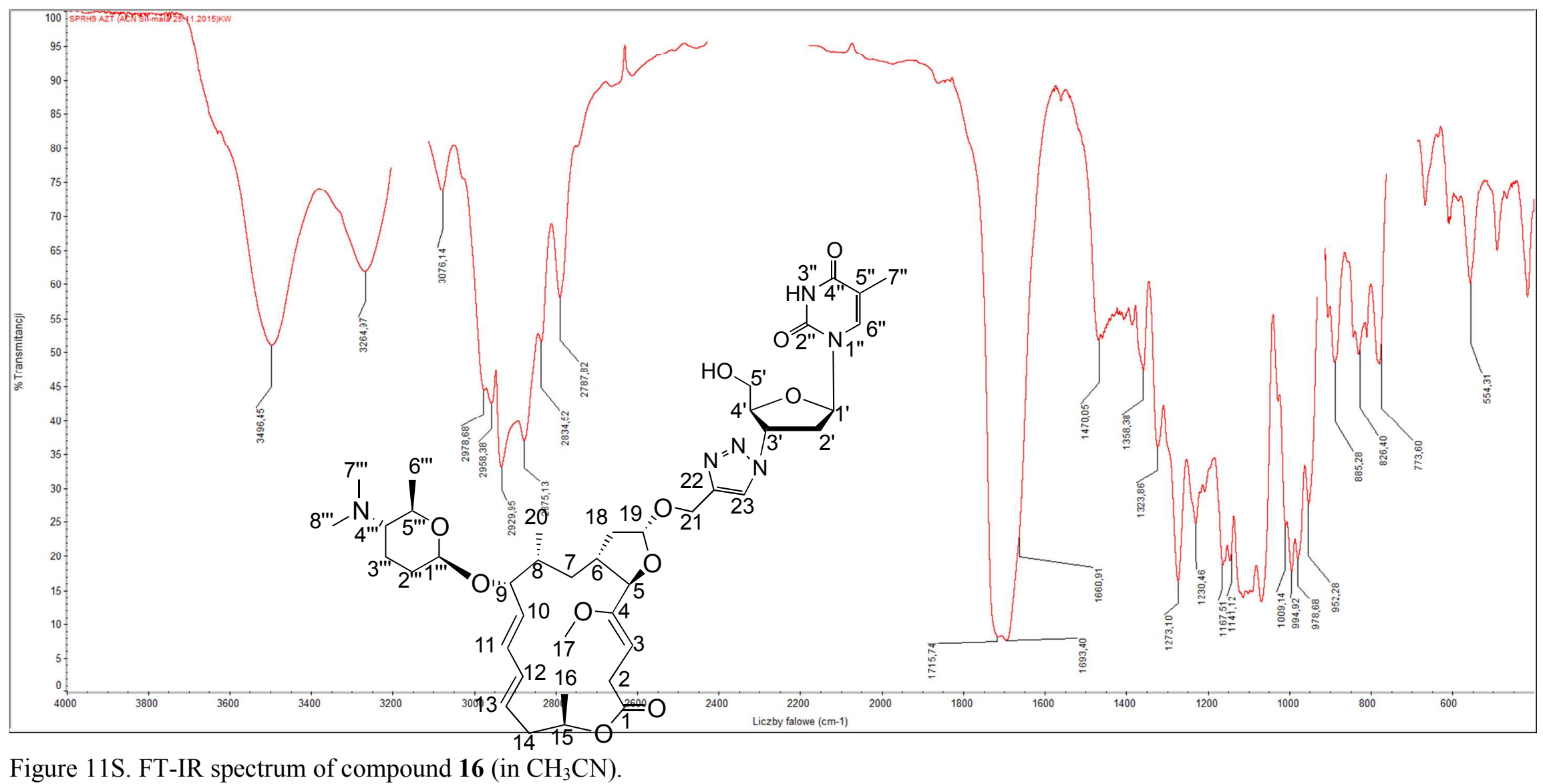

Figure 11S. FT-IR spectrum of compound 16 (in $\mathrm{CH}_{3} \mathrm{CN}$ ).

S15 


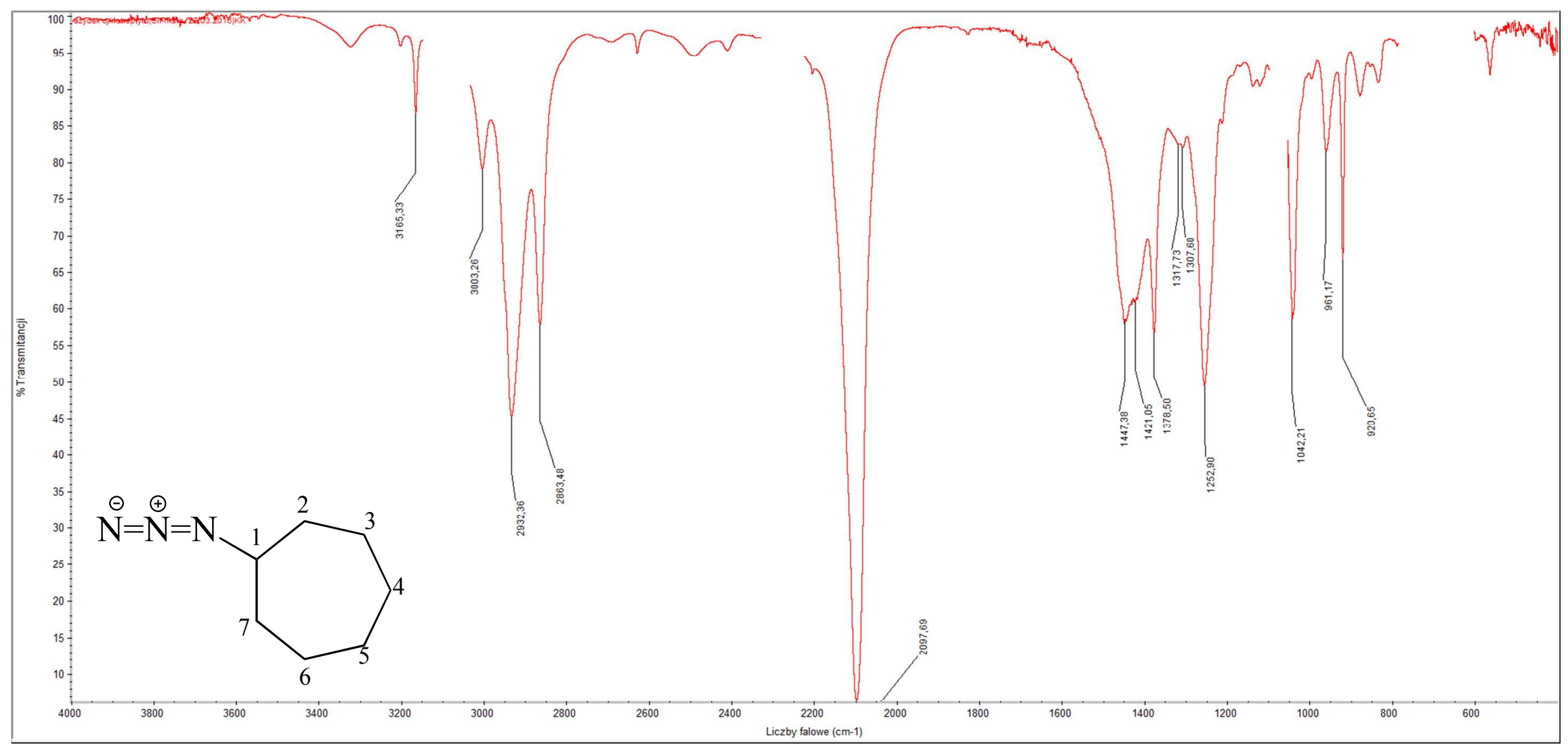

Figure 12S. FT-IR spectrum of compound $\mathbf{A Z 1}$ (in $\mathrm{CH}_{3} \mathrm{CN}$ ). 


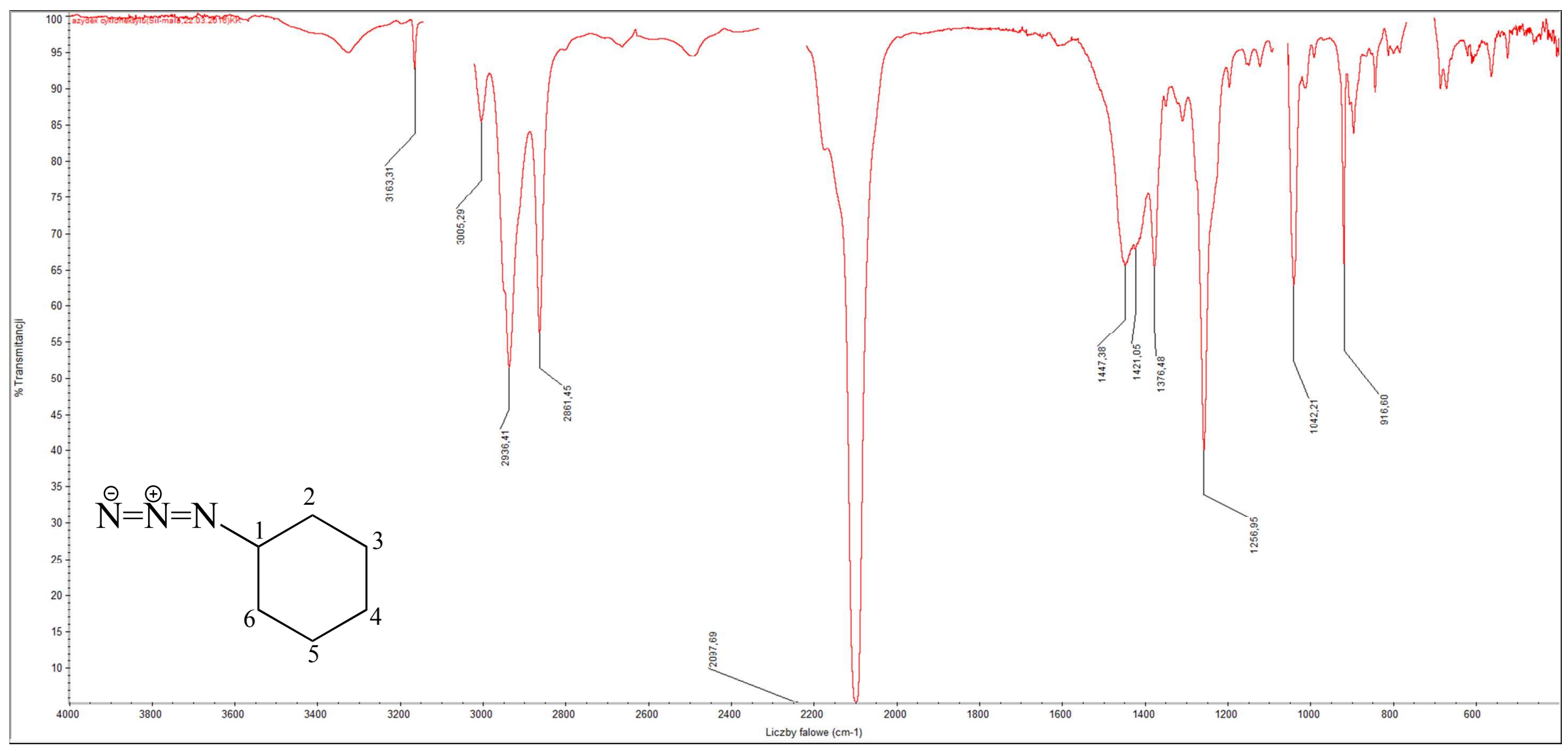

Figure 13S. FT-IR spectrum of compound $\mathbf{A Z 2}$ (in $\mathrm{CH}_{3} \mathrm{CN}$ ).

S17 


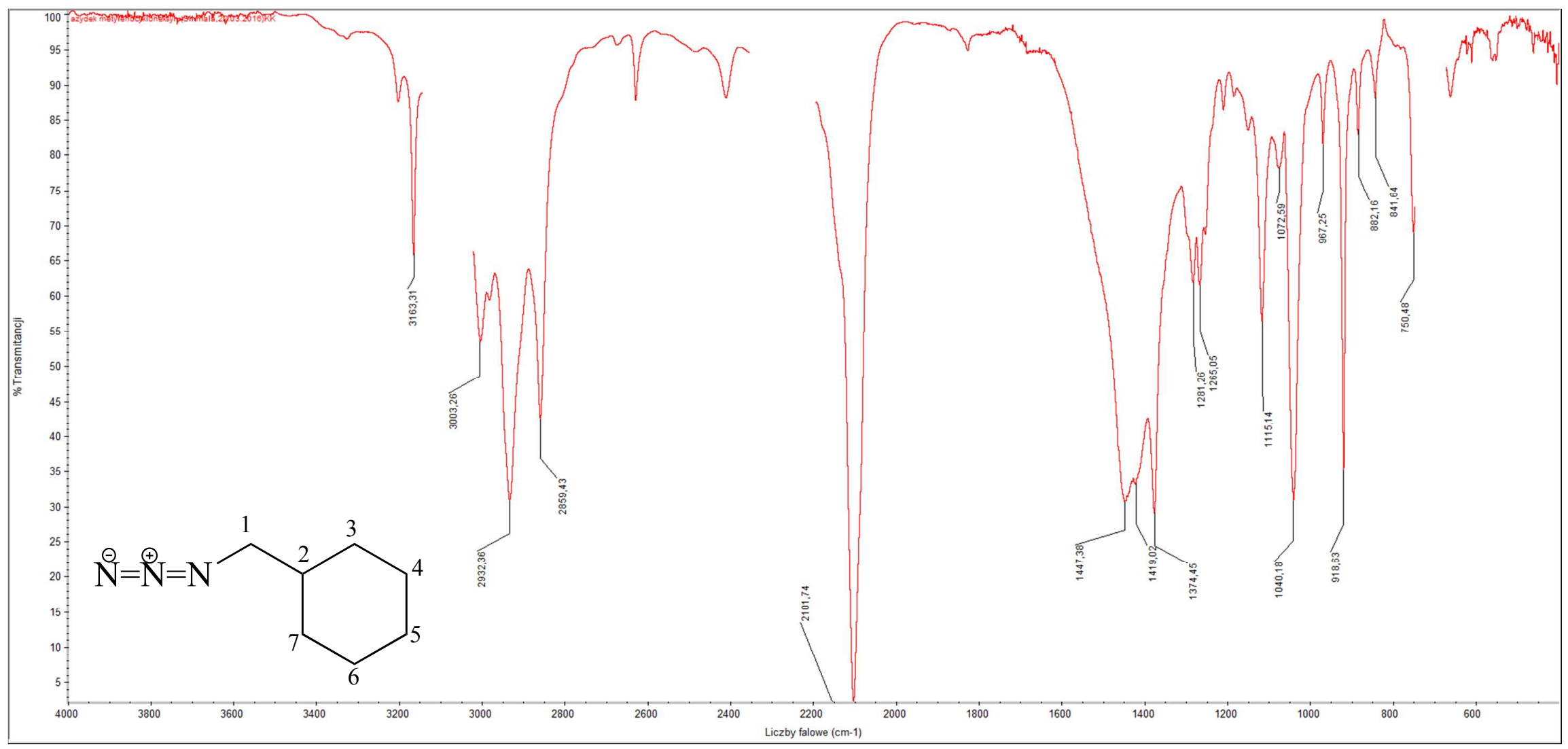

Figure 14S. FT-IR spectrum of compound $\mathbf{A Z 3}$ (in $\mathrm{CH}_{3} \mathrm{CN}$ ).

S18 


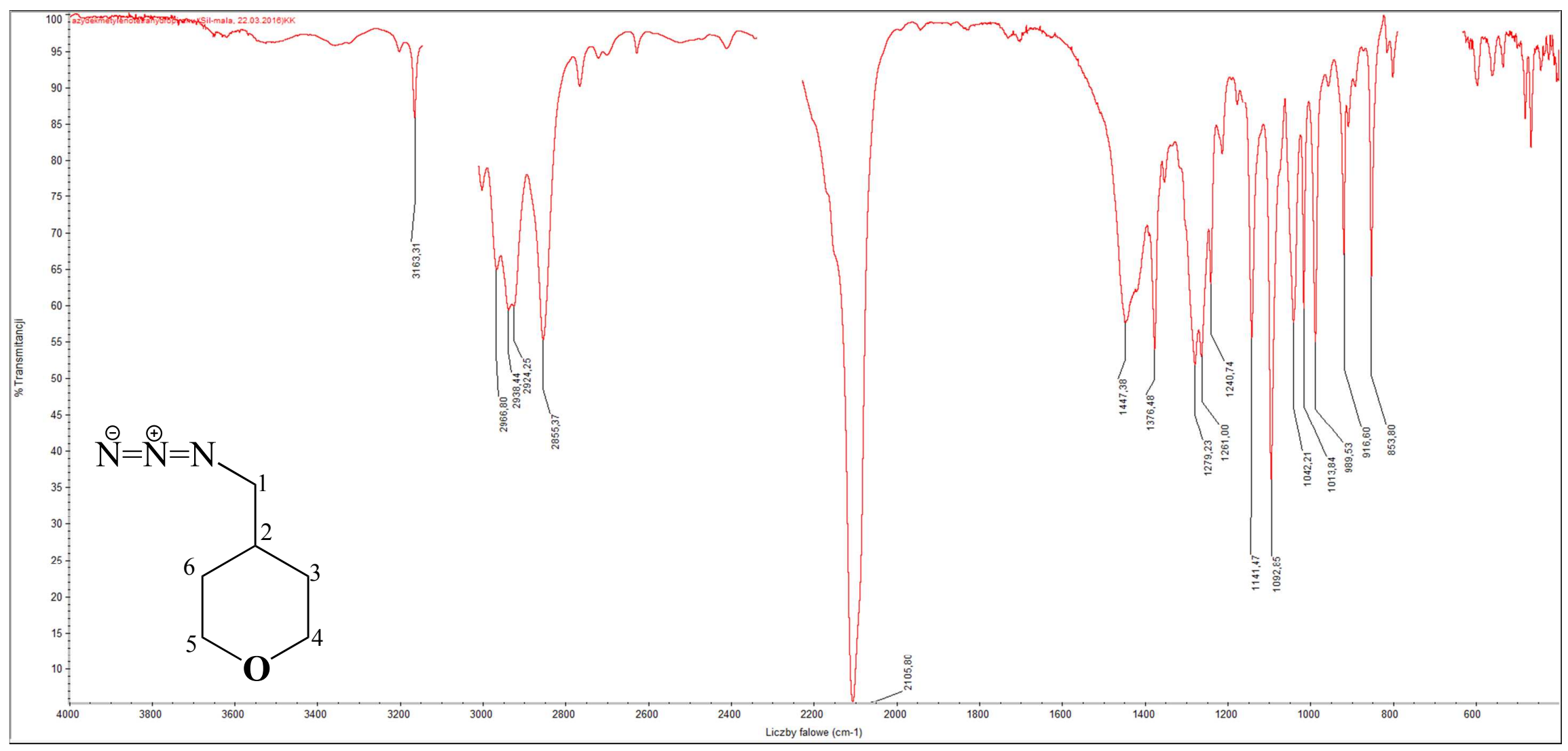

Figure 15S. FT-IR spectrum of compound $\mathbf{A Z 4}$ (in $\mathrm{CH}_{3} \mathrm{CN}$ ).

S19 


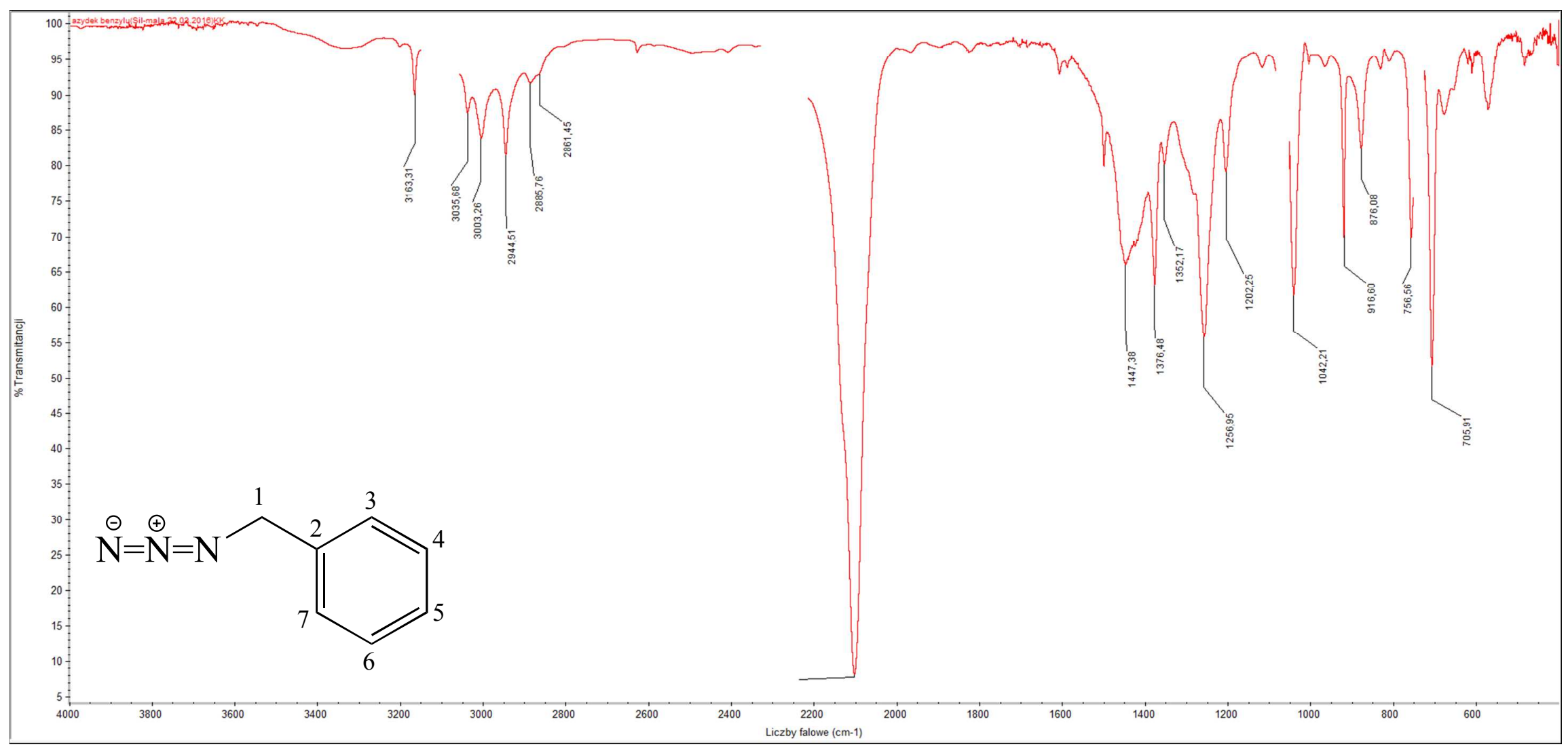

Figure 16S. FT-IR spectrum of compound $\mathbf{A Z 5}$ (in $\mathrm{CH}_{3} \mathrm{CN}$ ).

S20 


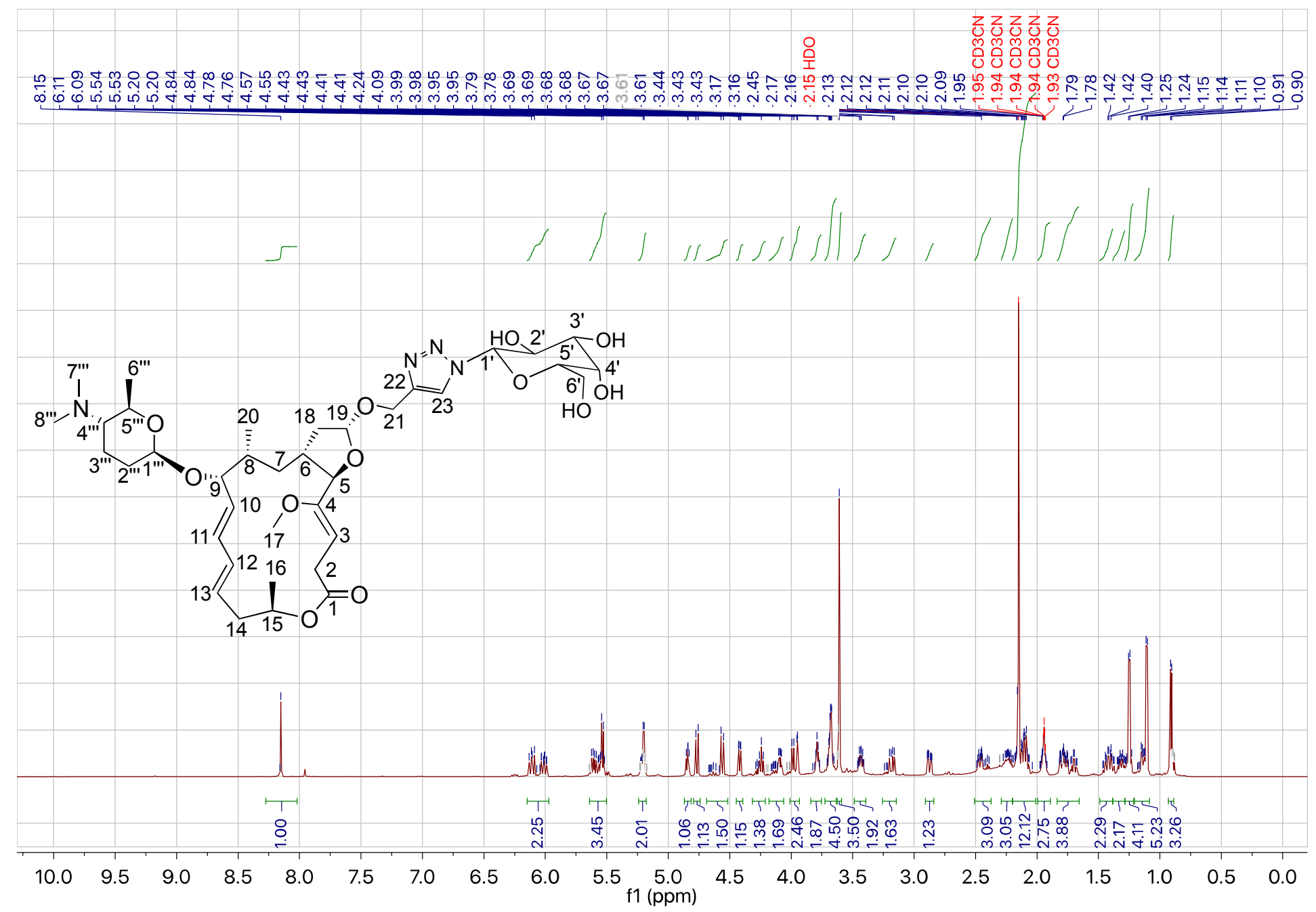

Figure 17S. ${ }^{1} \mathrm{H}$ NMR spectrum of compound 6 in $\mathrm{CD}_{3} \mathrm{CN}$. 


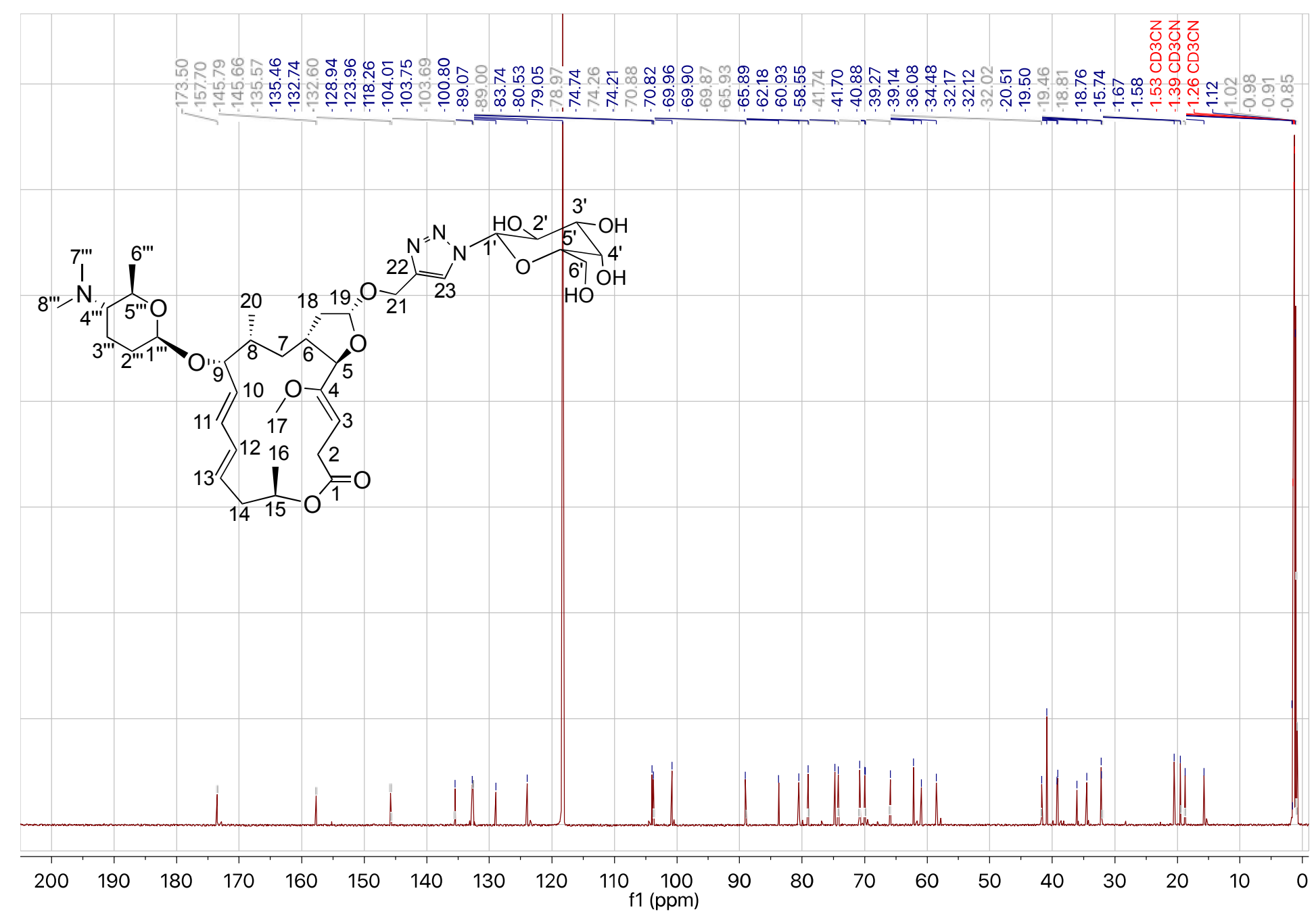

Figure $18 \mathrm{~S} .{ }^{13} \mathrm{C}$ NMR spectrum of compound 6 in $\mathrm{CD}_{3} \mathrm{CN}$. 


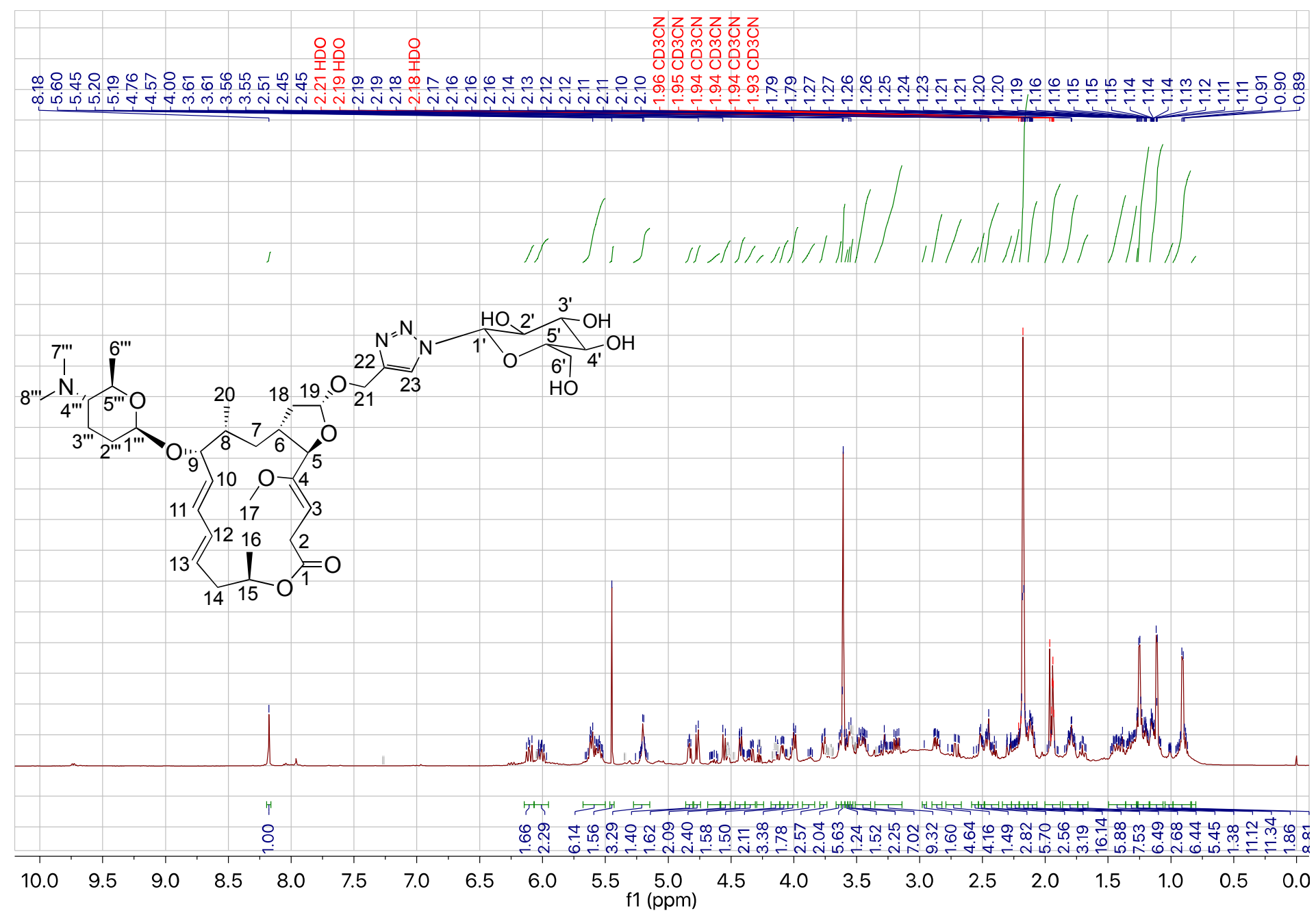

Figure 19S. ${ }^{1} \mathrm{H}$ NMR spectrum of compound 7 in $\mathrm{CD}_{3} \mathrm{CN}$. 


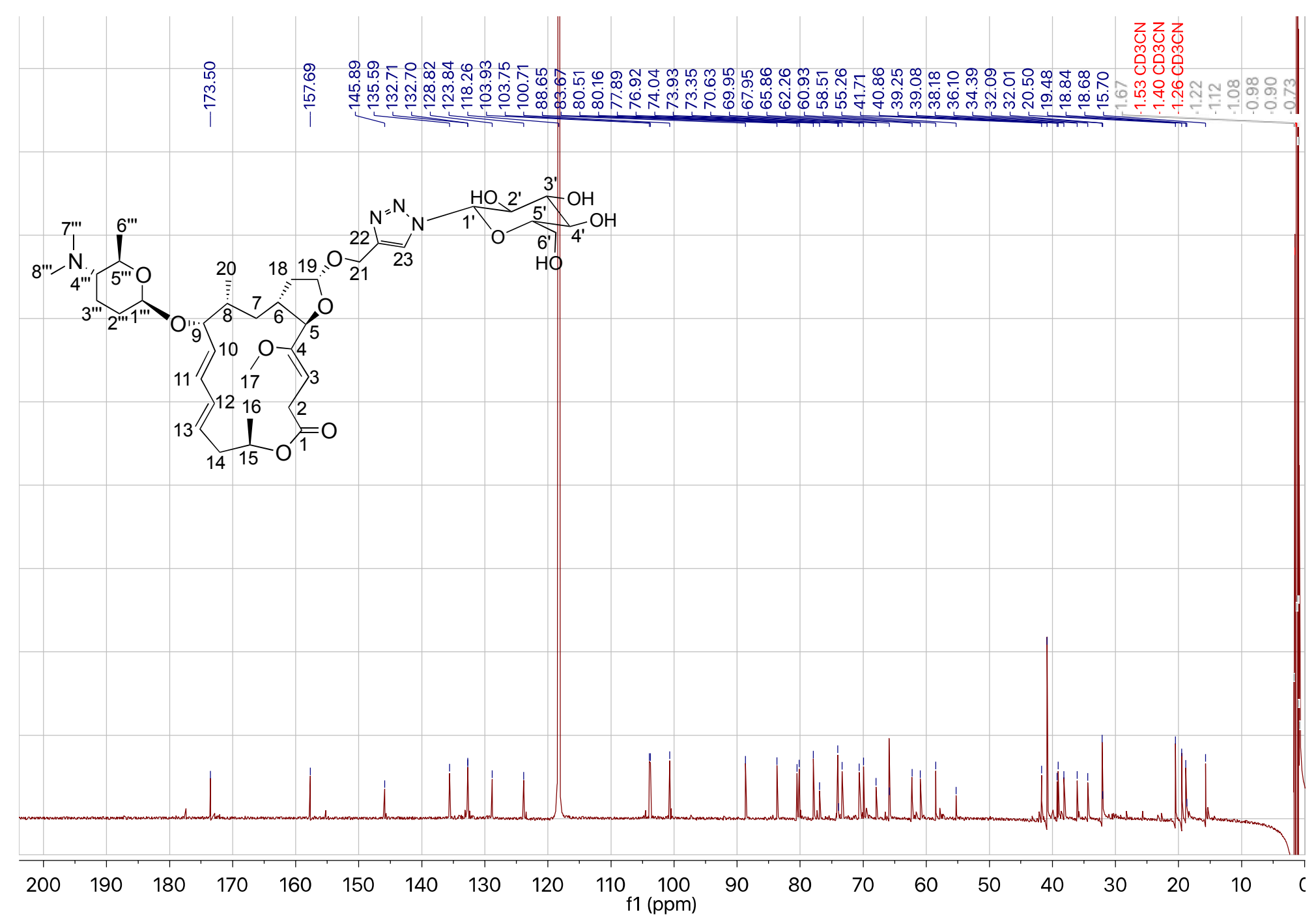

Figure 20S. ${ }^{13} \mathrm{C}$ NMR spectrum of compound 7 in $\mathrm{CD}_{3} \mathrm{CN}$. 


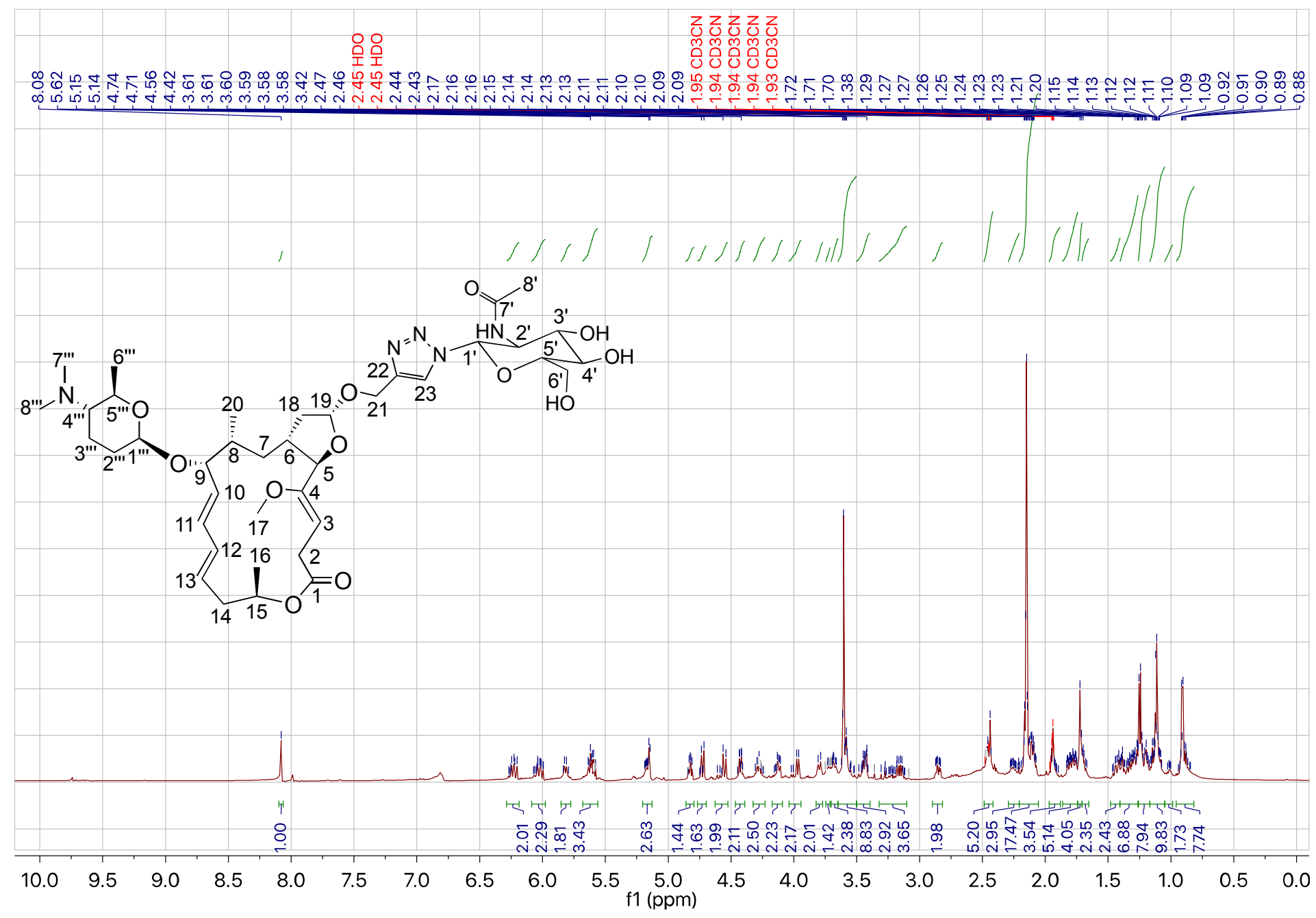

Figure 21S. ${ }^{1} \mathrm{H}$ NMR spectrum of compound 8 in $\mathrm{CD}_{3} \mathrm{CN}$. 


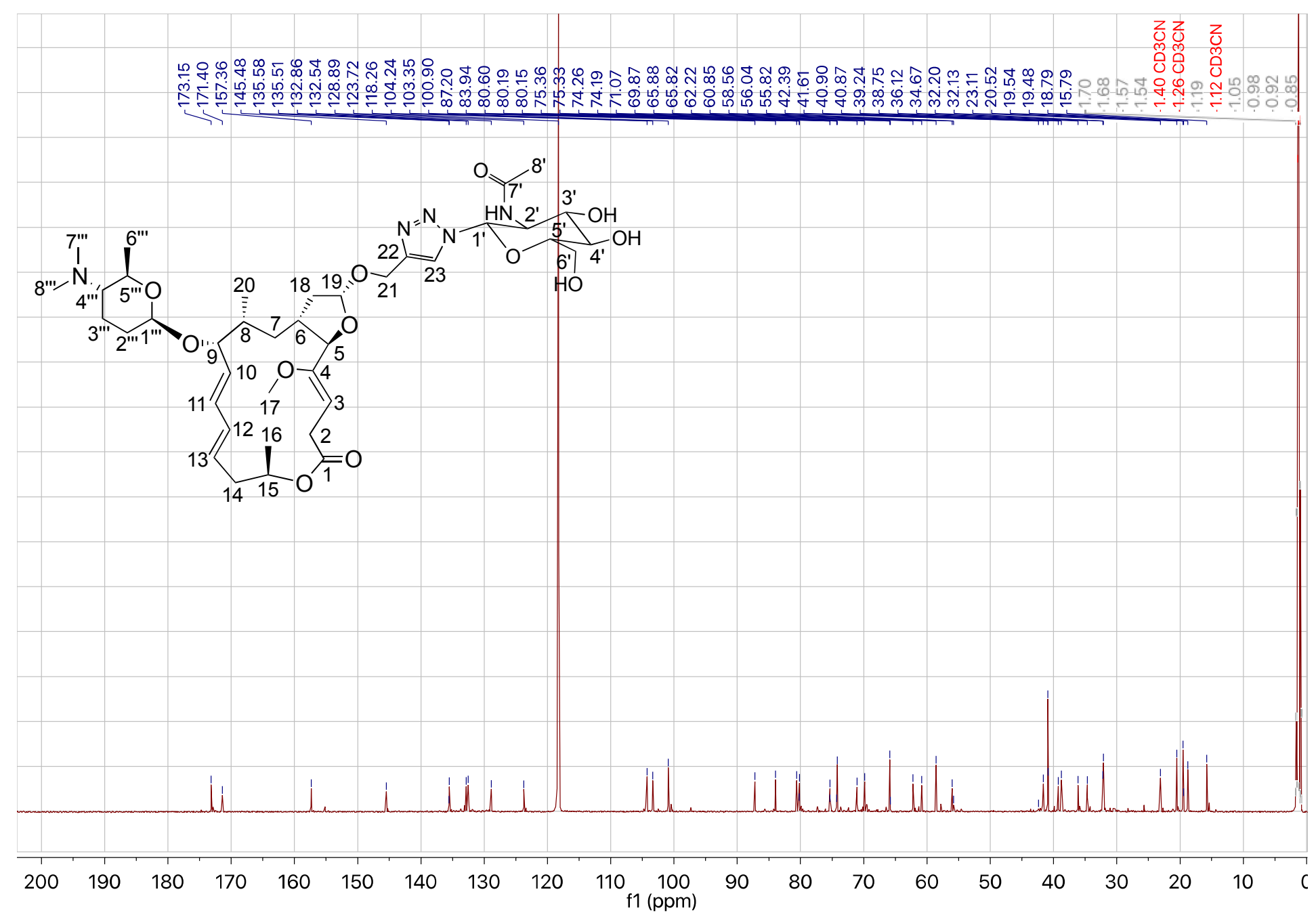

Figure $22 \mathrm{~S} .{ }^{13} \mathrm{C}$ NMR spectrum of compound $\mathbf{8}$ in $\mathrm{CD}_{3} \mathrm{CN}$. 


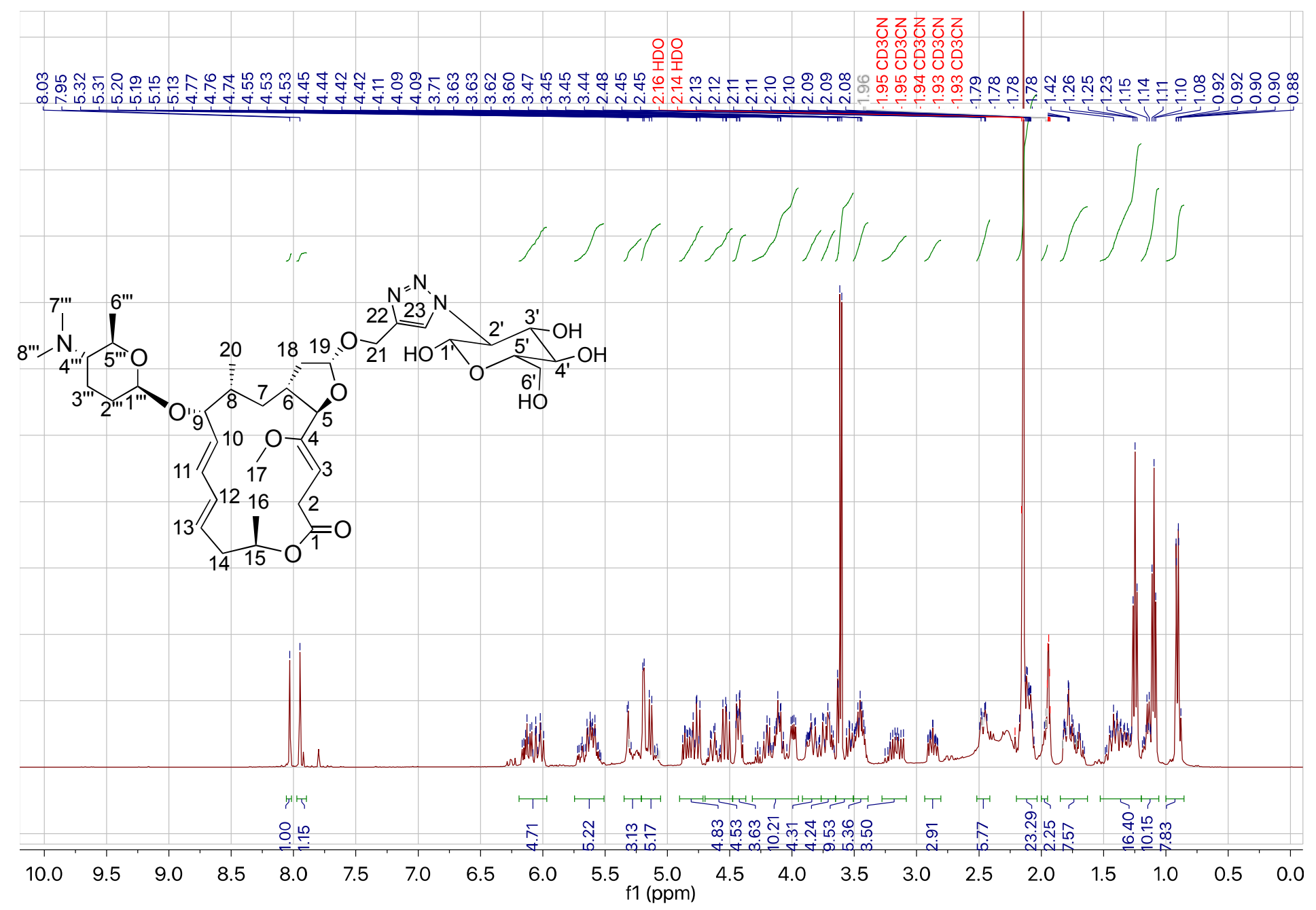

Figure 23S. ${ }^{1} \mathrm{H}$ NMR spectrum of compound 9 in $\mathrm{CD}_{3} \mathrm{CN}$. 


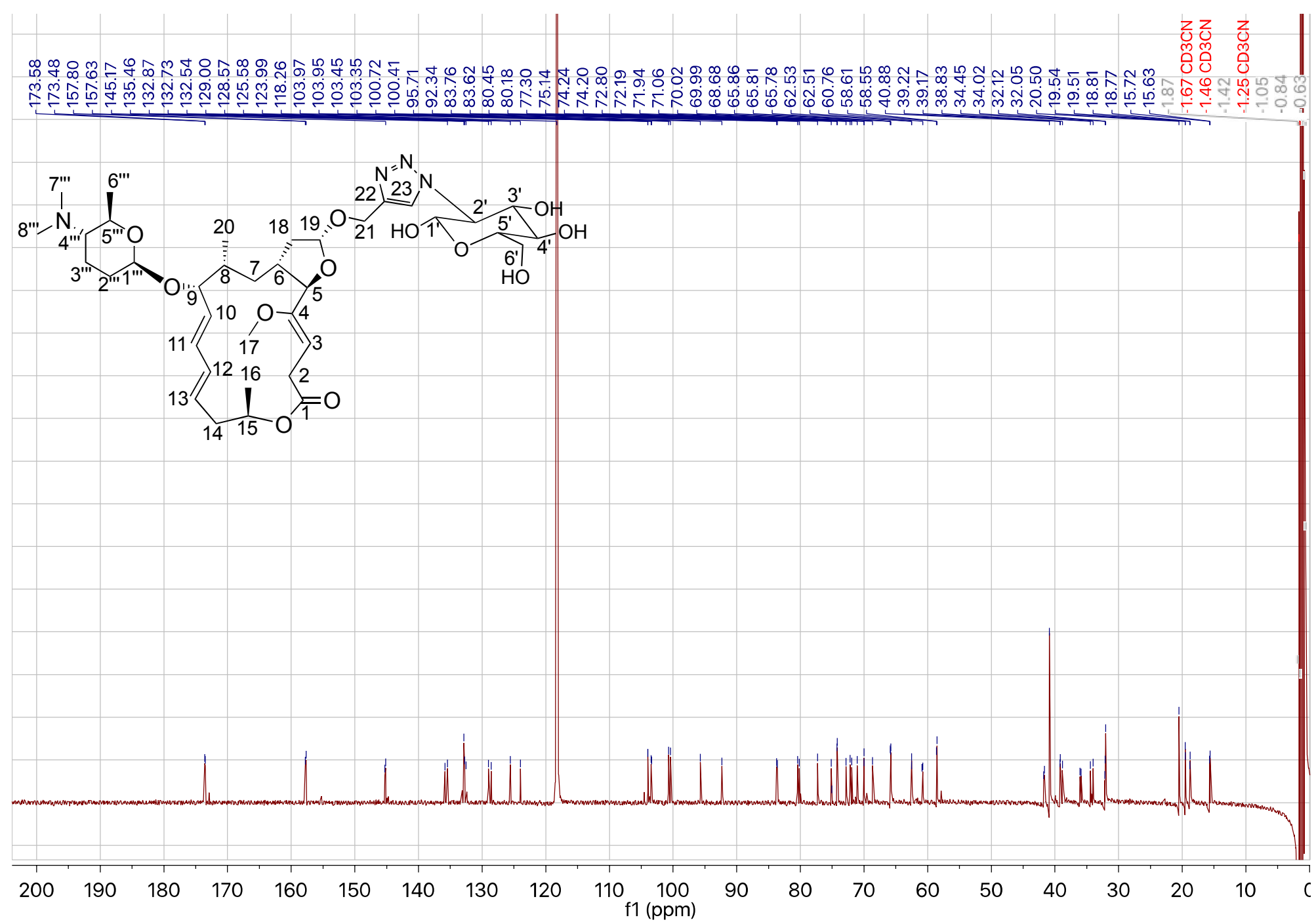

Figure 24S. ${ }^{13} \mathrm{C}$ NMR spectrum of compound 9 in $\mathrm{CD}_{3} \mathrm{CN}$. 


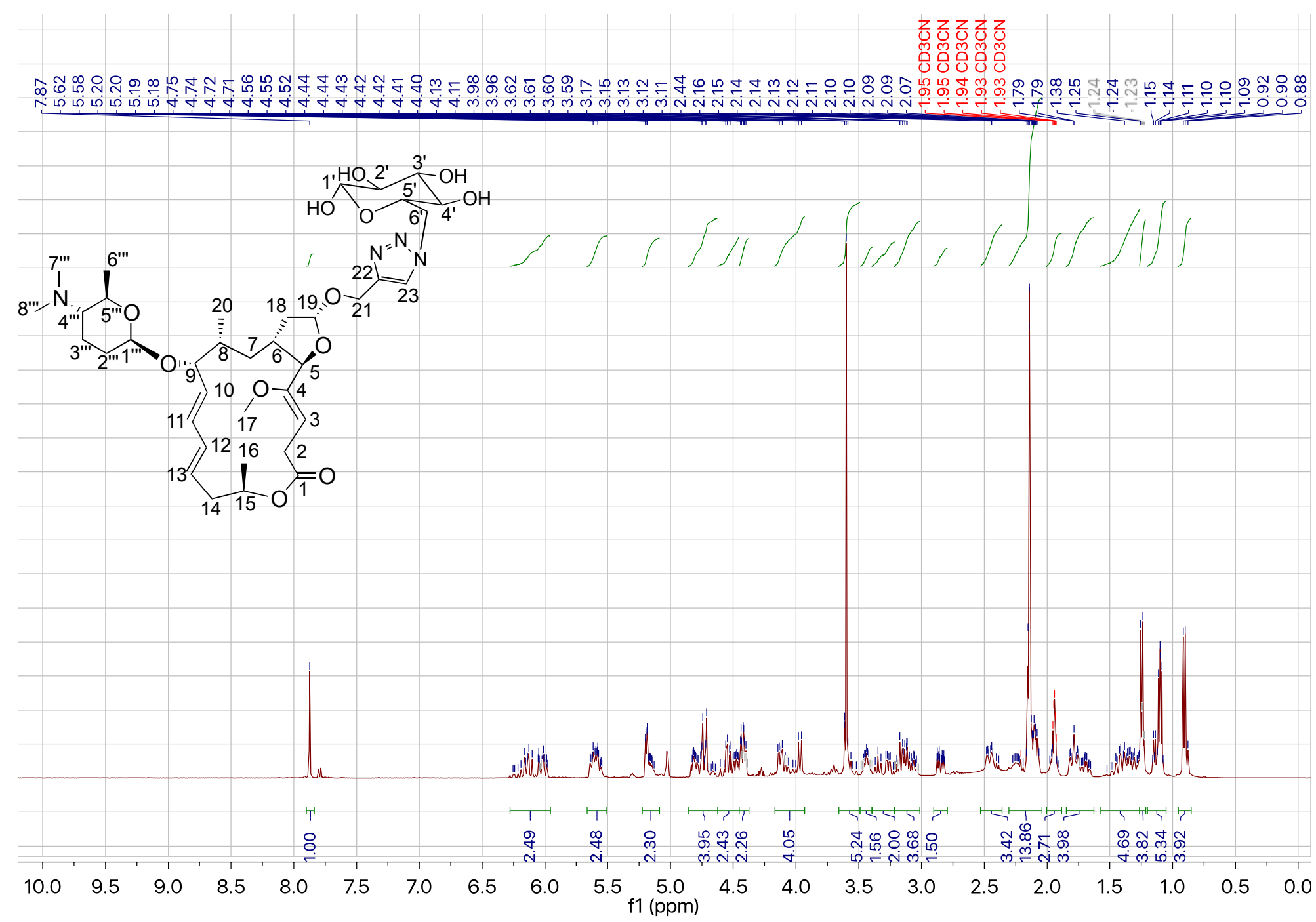

Figure 25S. ${ }^{1} \mathrm{H}$ NMR spectrum of compound $\mathbf{1 0}$ in $\mathrm{CD}_{3} \mathrm{CN}$. 
구의

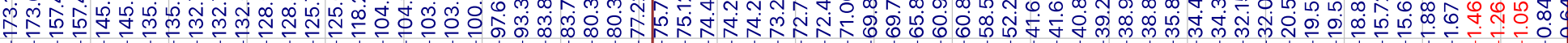
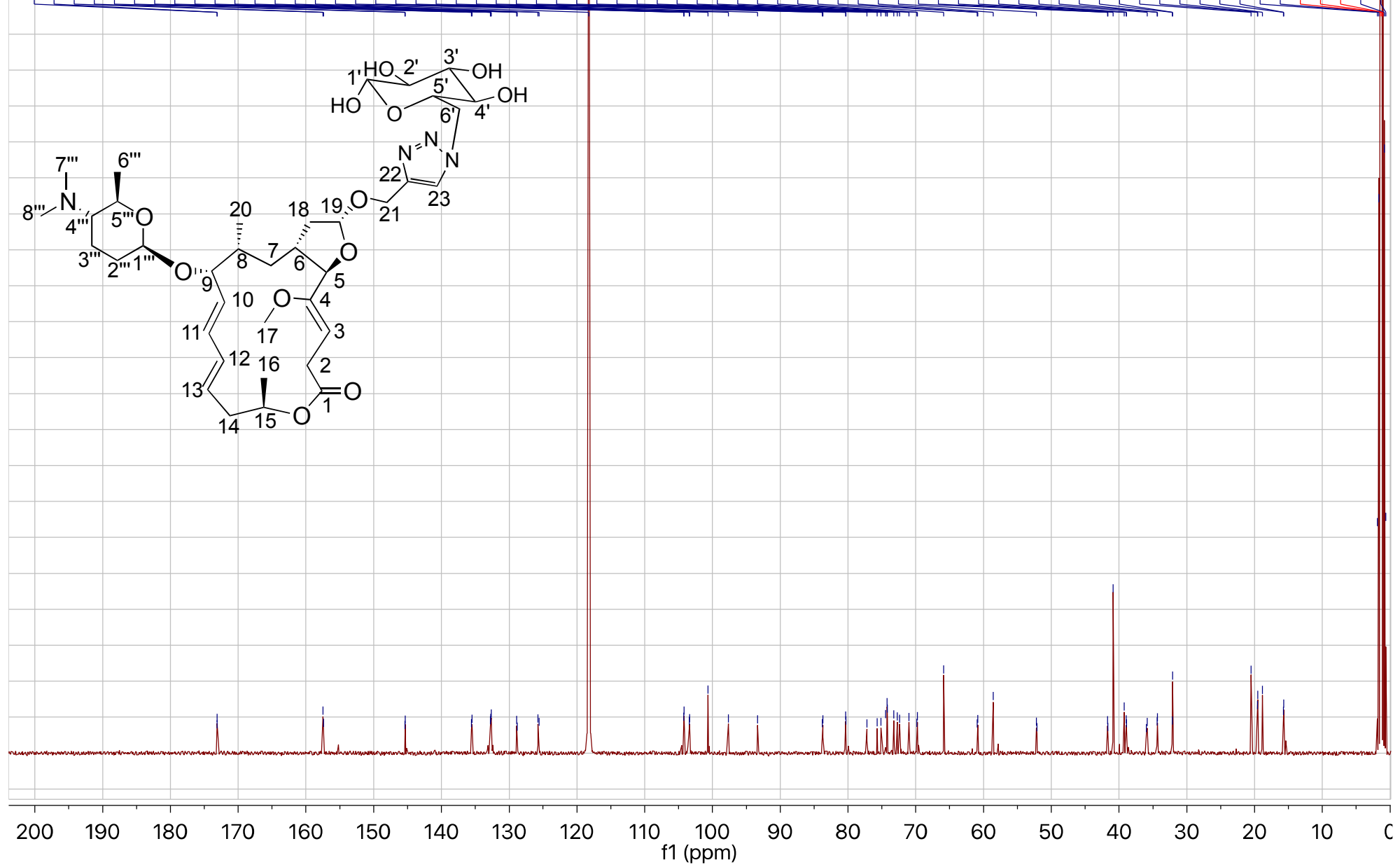

Figure 26S. ${ }^{13} \mathrm{C}$ NMR spectrum of compound 10 in $\mathrm{CD}_{3} \mathrm{CN}$. 


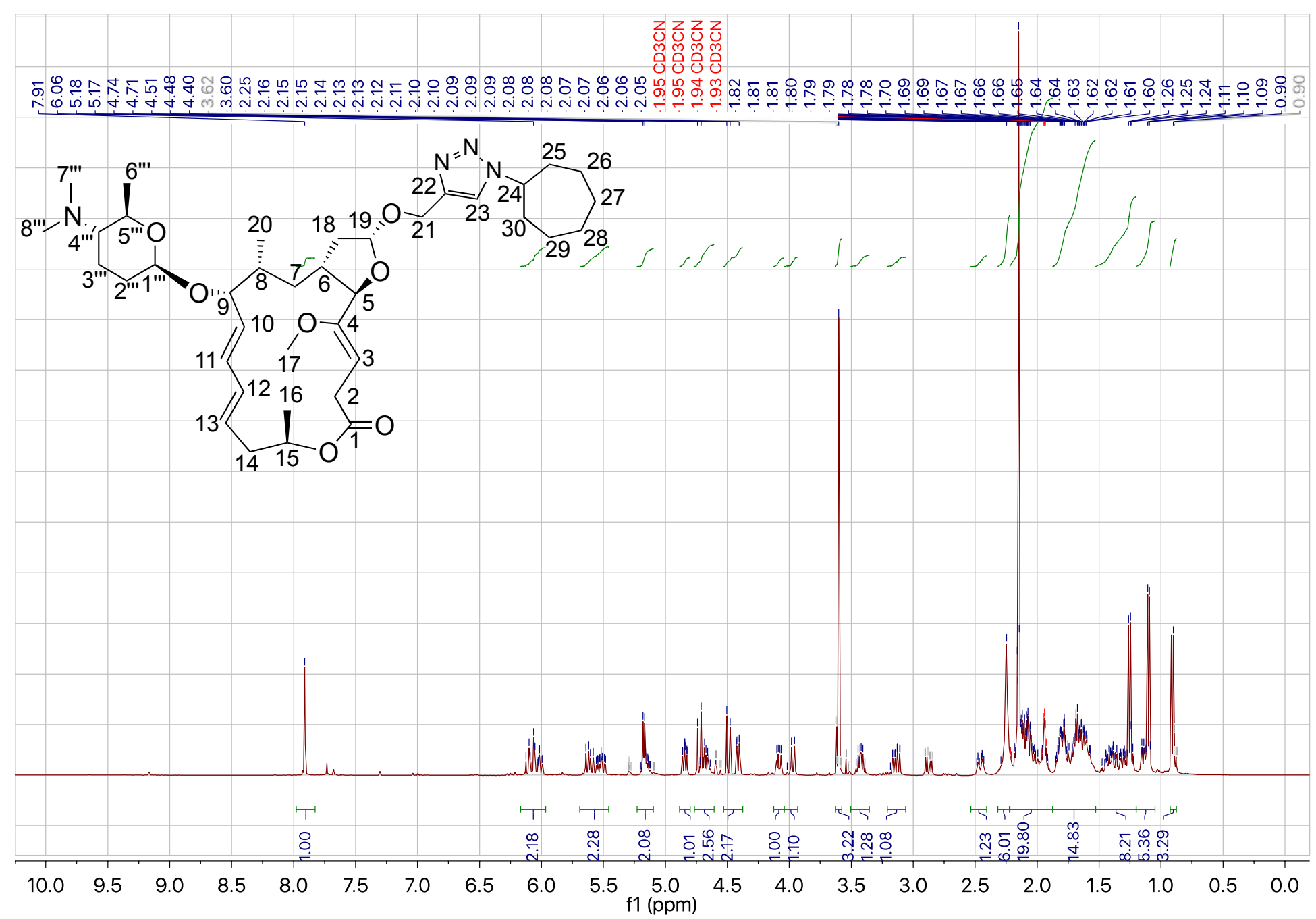

Figure 27S. ${ }^{1} \mathrm{H}$ NMR spectrum of compound 11 in $\mathrm{CD}_{3} \mathrm{CN}$. 


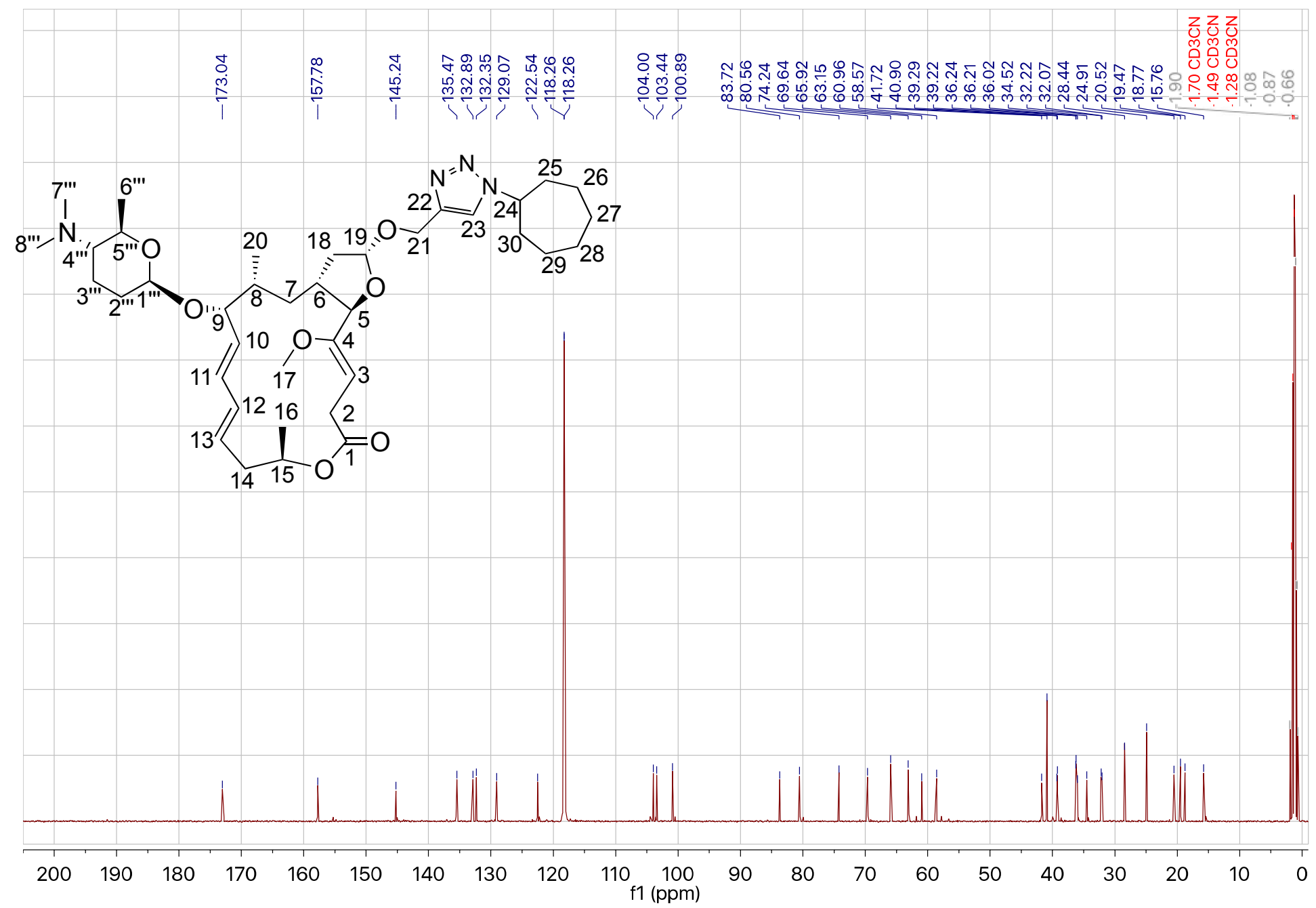

Figure 28S. ${ }^{13} \mathrm{C}$ NMR spectrum of compound 11 in $\mathrm{CD}_{3} \mathrm{CN}$. 


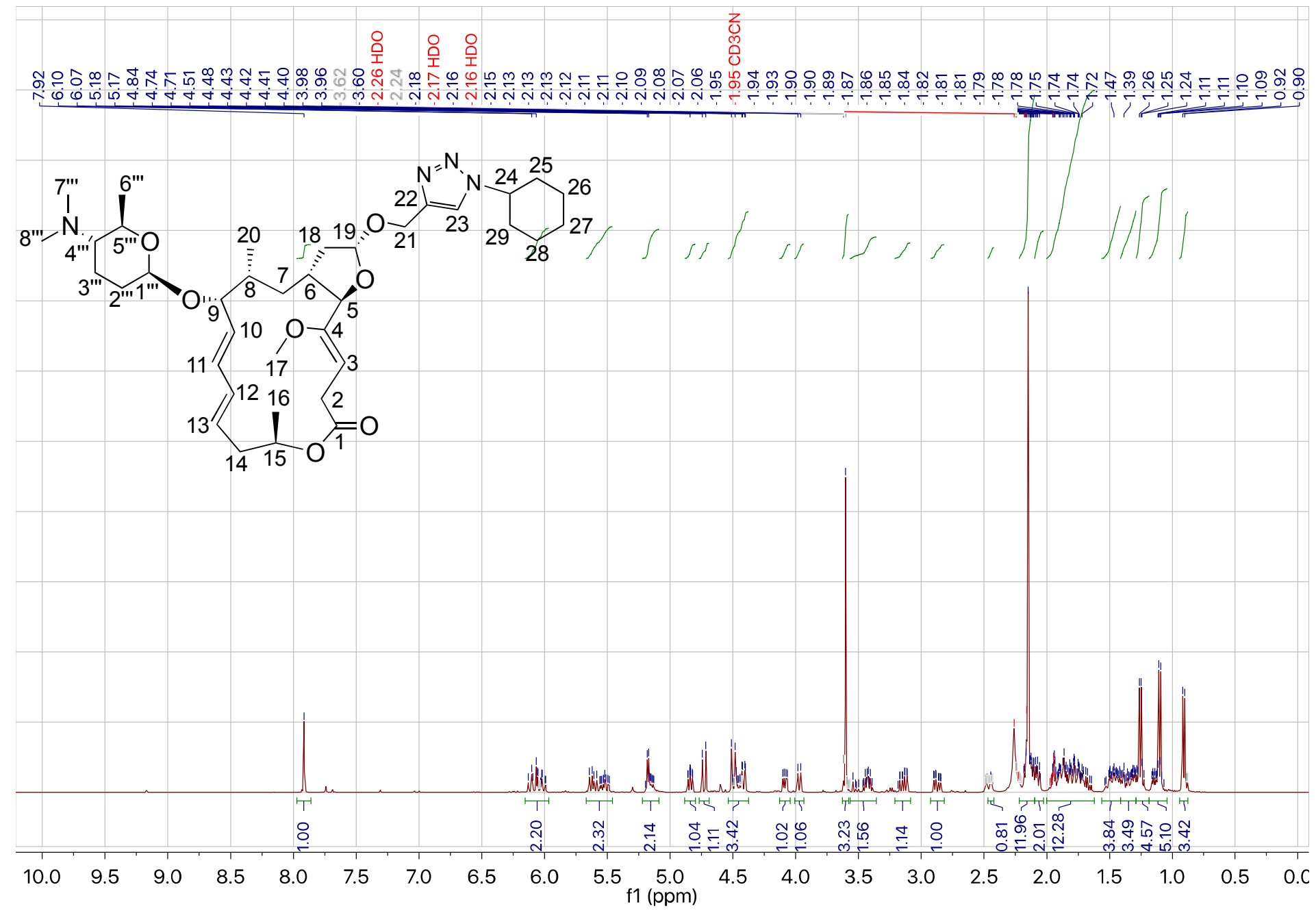

Figure 29S. ${ }^{1} \mathrm{H}$ NMR spectrum of compound 12 in $\mathrm{CD}_{3} \mathrm{CN}$. 


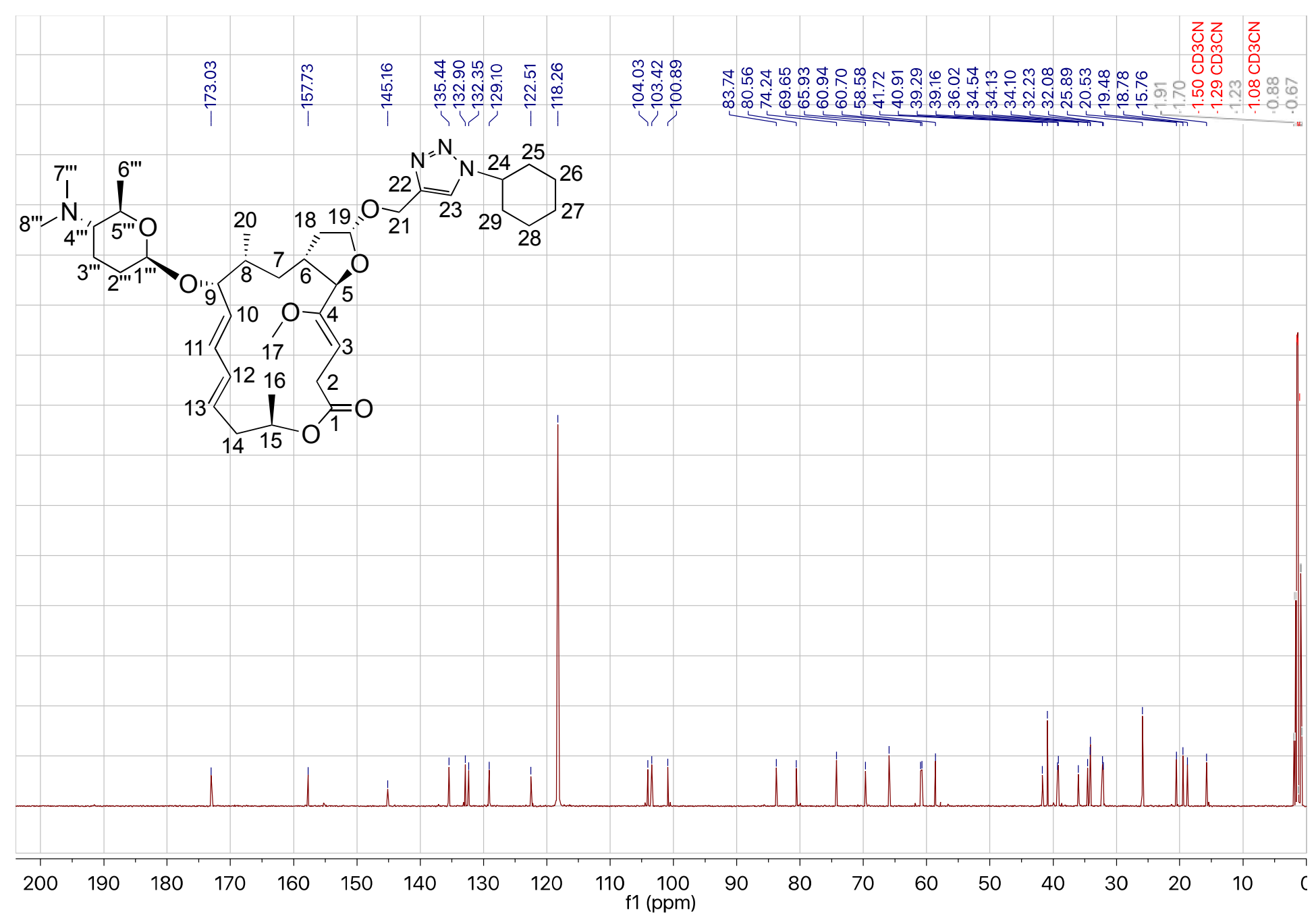

Figure $30 \mathrm{~S} .{ }^{13} \mathrm{C}$ NMR spectrum of compound $\mathbf{1 2}$ in $\mathrm{CD}_{3} \mathrm{CN}$. 


\section{오 웅}

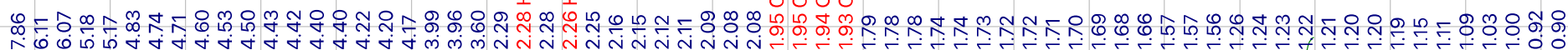

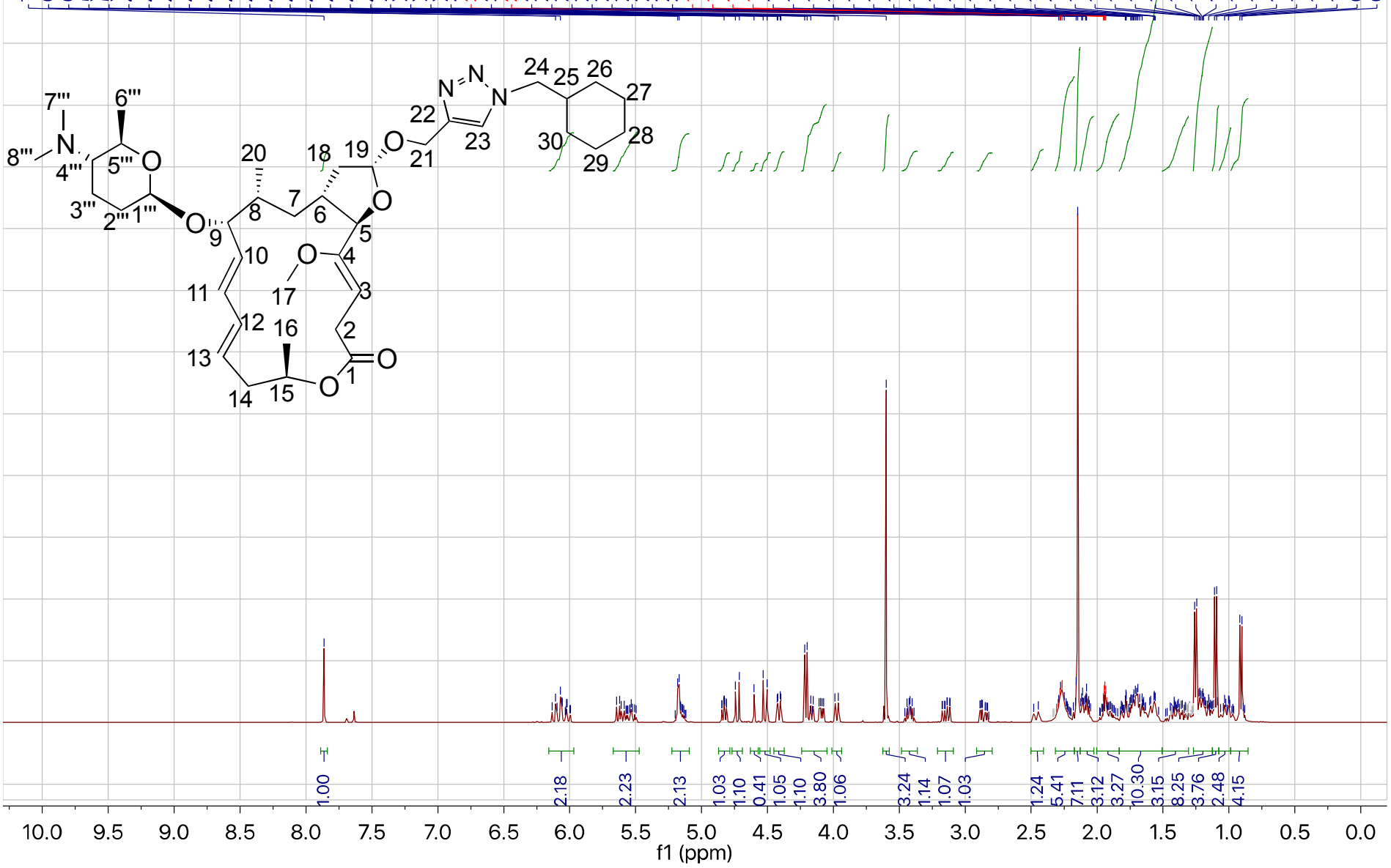

Figure $31 \mathrm{~S} .{ }^{1} \mathrm{H}$ NMR spectrum of compound 13 in $\mathrm{CD}_{3} \mathrm{CN}$. 


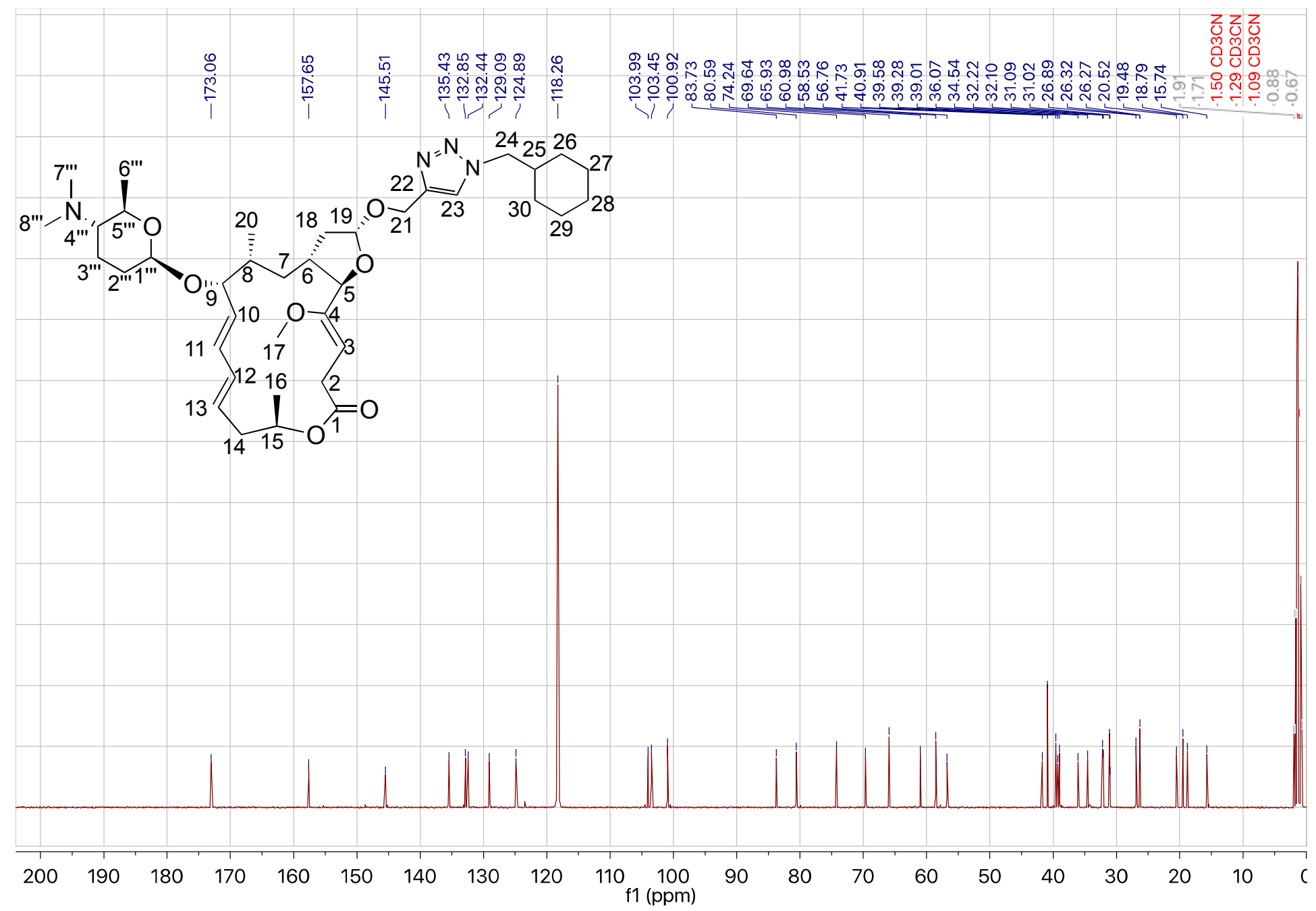

Figure $32 \mathrm{~S} .{ }^{13} \mathrm{C}$ NMR spectrum of compound $\mathbf{1 3}$ in $\mathrm{CD}_{3} \mathrm{CN}$. 


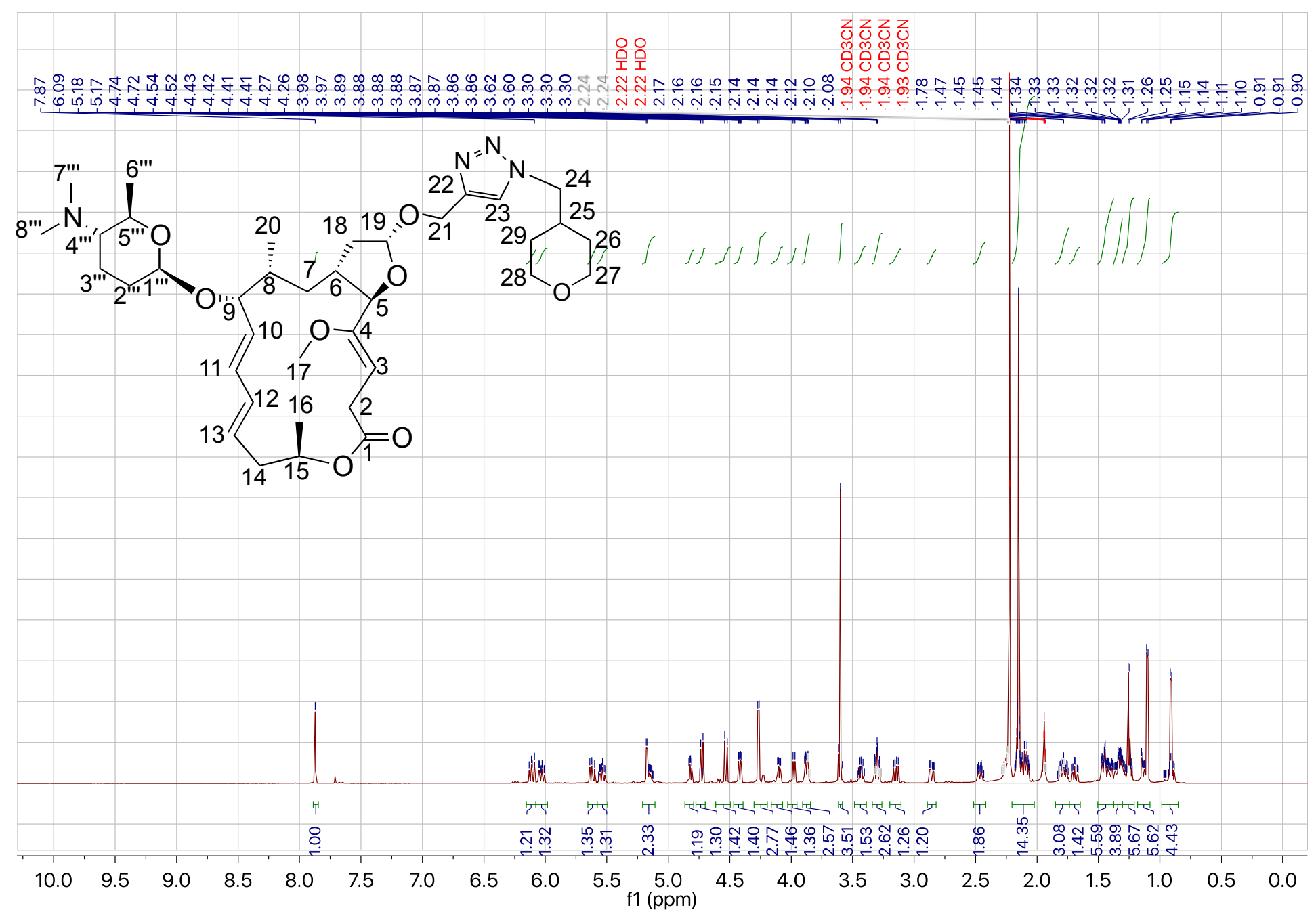

Figure 33S. ${ }^{1} \mathrm{H}$ NMR spectrum of compound 14 in $\mathrm{CD}_{3} \mathrm{CN}$. 


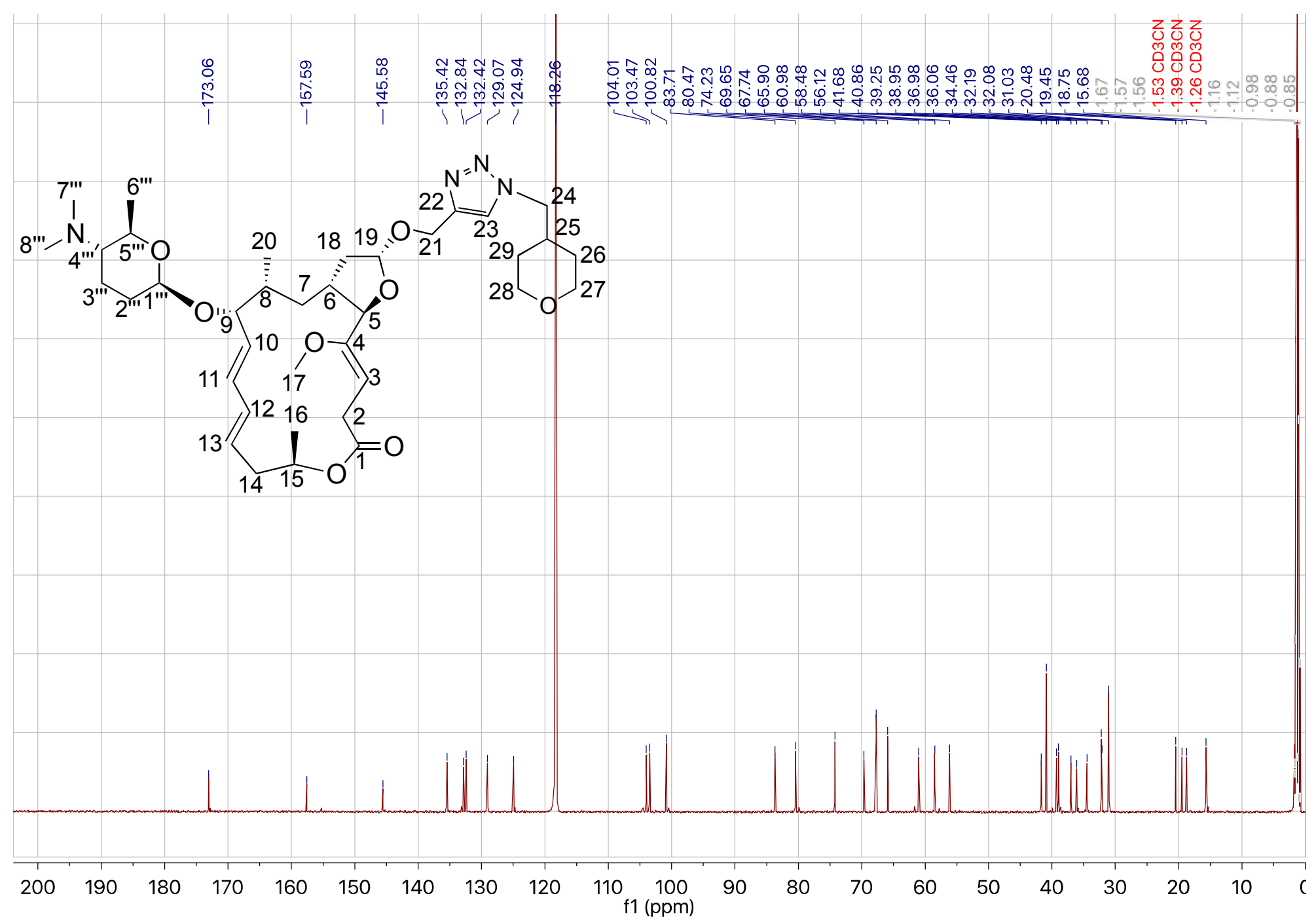

Figure $34 \mathrm{~S} .{ }^{13} \mathrm{C}$ NMR spectrum of compound 14 in $\mathrm{CD}_{3} \mathrm{CN}$. 


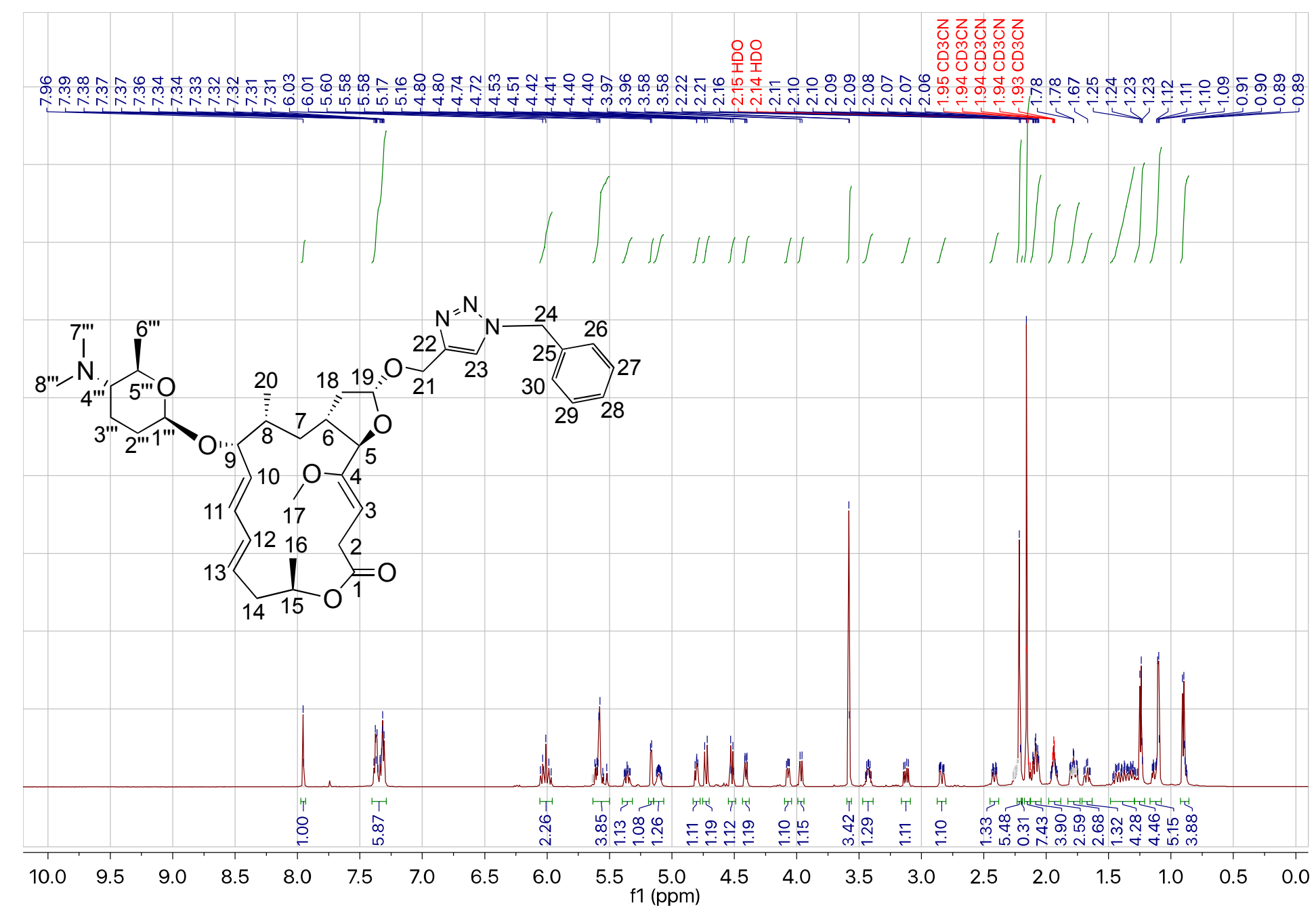

Figure 35S. ${ }^{1} \mathrm{H}$ NMR spectrum of compound 15 in $\mathrm{CD}_{3} \mathrm{CN}$. 


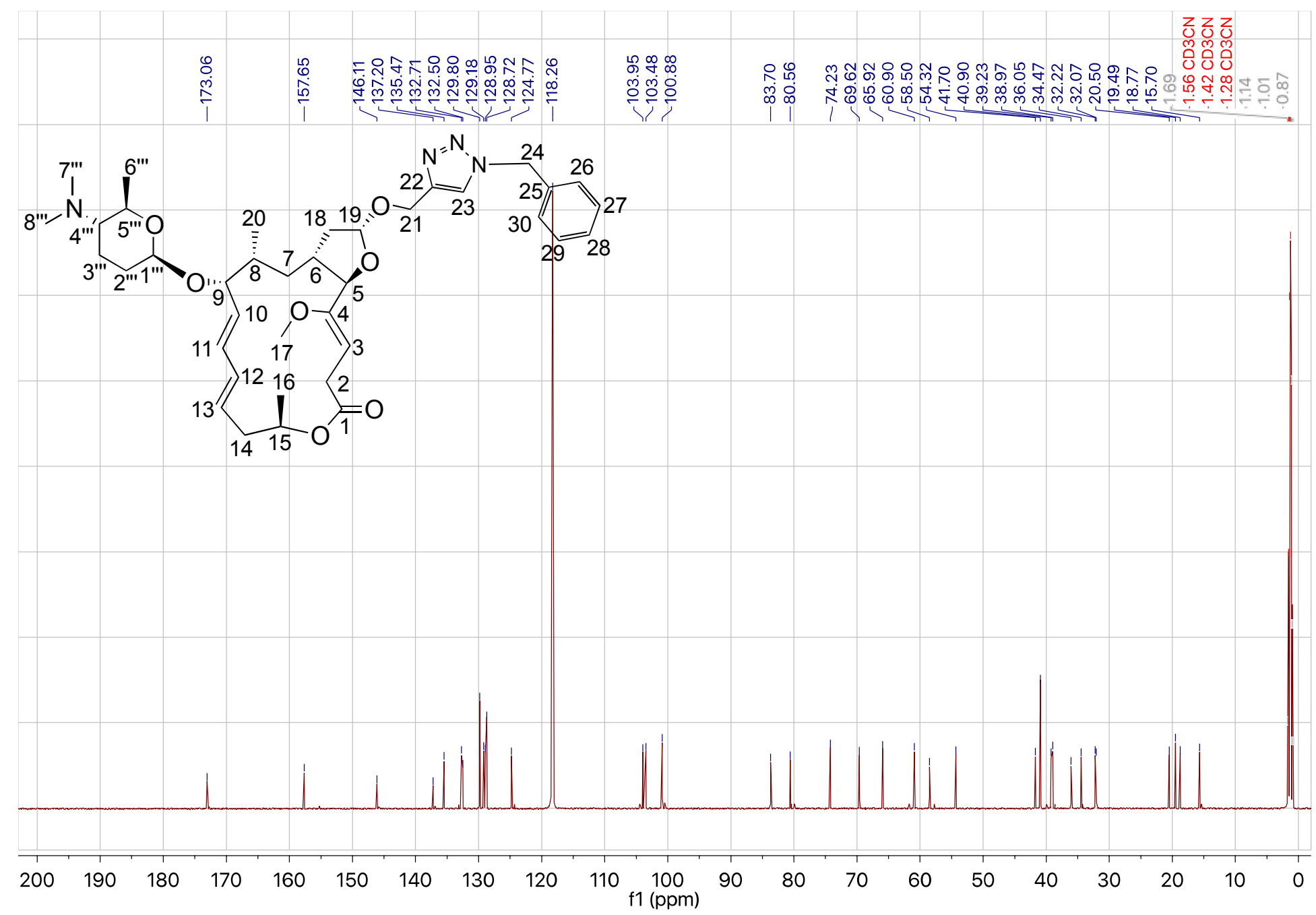

Figure $36 \mathrm{~S} .{ }^{13} \mathrm{C}$ NMR spectrum of compound 15 in $\mathrm{CD}_{3} \mathrm{CN}$. 


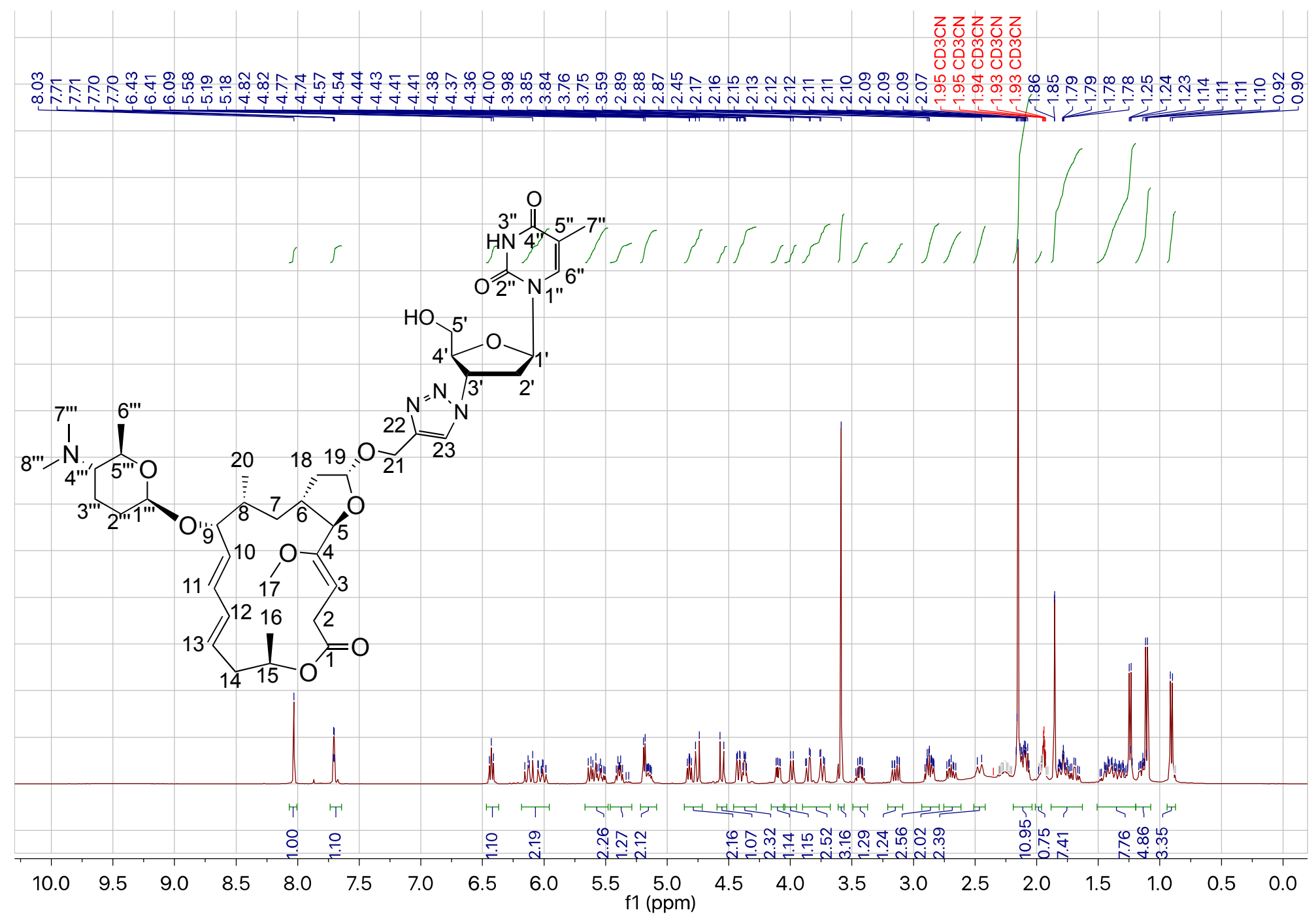

Figure 37S. ${ }^{1} \mathrm{H}$ NMR spectrum of compound 16 in $\mathrm{CD}_{3} \mathrm{CN}$. 


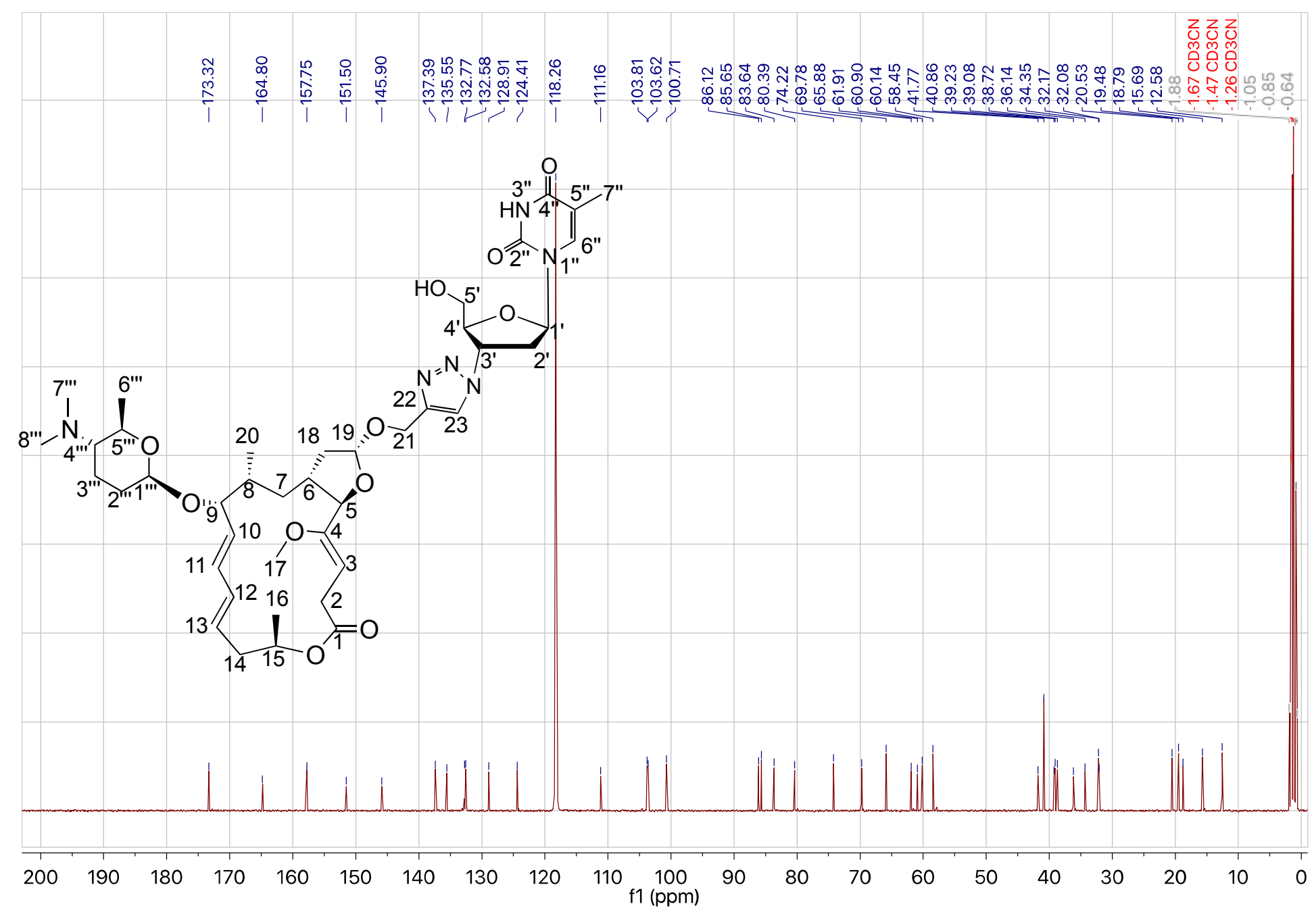

Figure $38 \mathrm{~S} .{ }^{13} \mathrm{C}$ NMR spectrum of compound 16 in $\mathrm{CD}_{3} \mathrm{CN}$. 


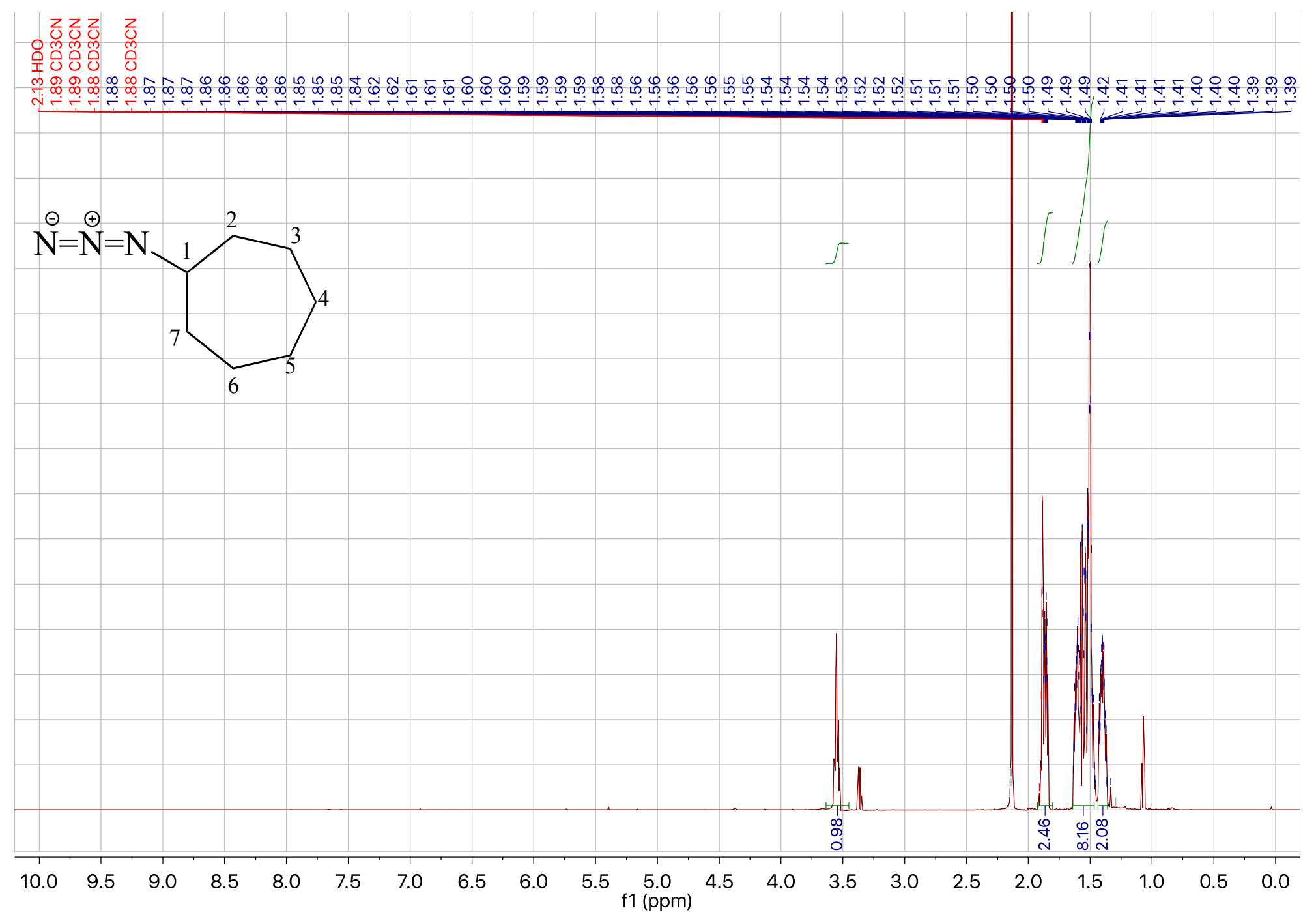

Figure 39S. ${ }^{1} \mathrm{H}$ NMR spectrum of compound $\mathbf{A Z 1}$ in $\mathrm{CD}_{3} \mathrm{CN}$. 


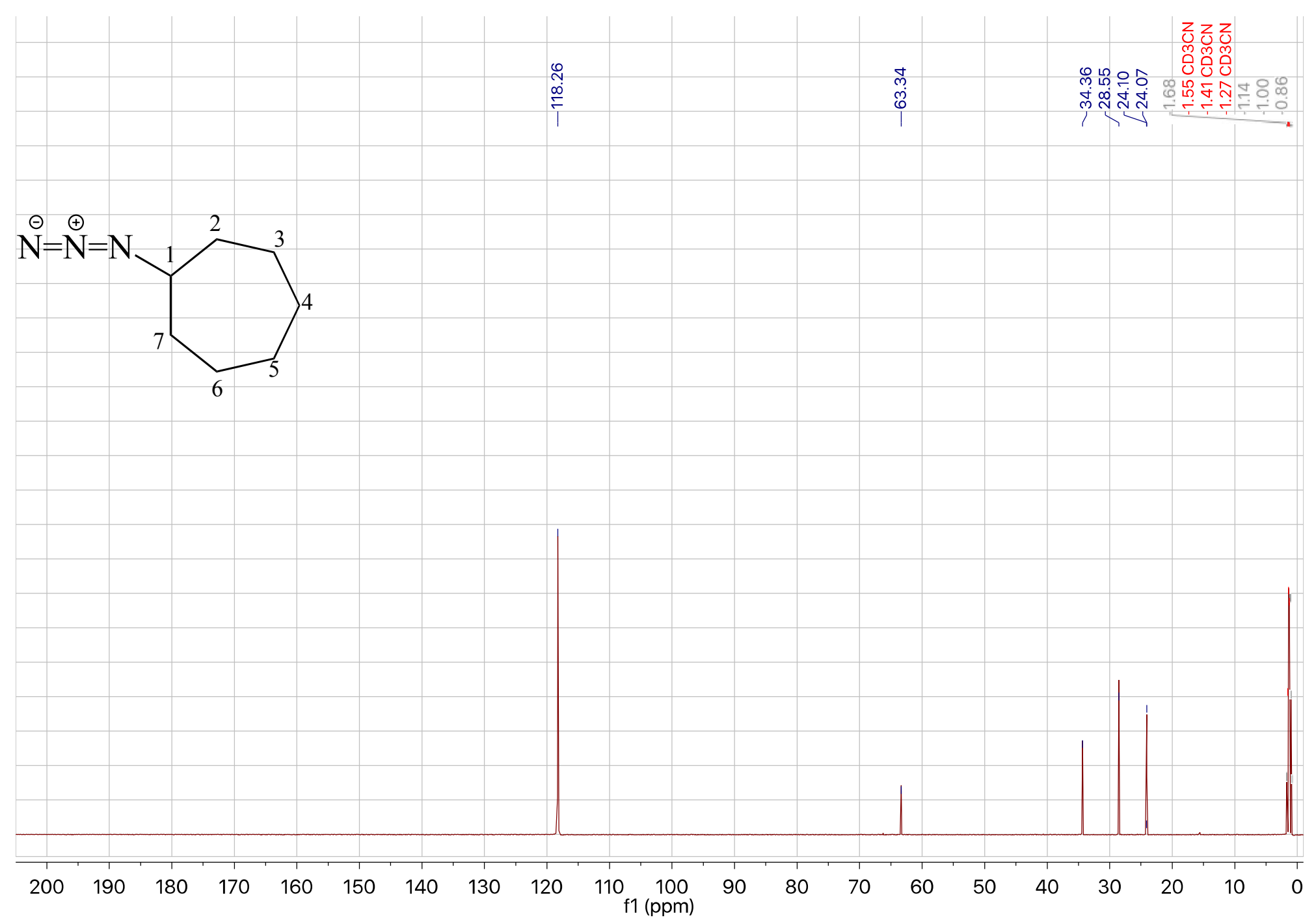

Figure 40S. ${ }^{13} \mathrm{C}$ NMR spectrum of compound $\mathbf{A Z 1}$ in $\mathrm{CD}_{3} \mathrm{CN}$. 


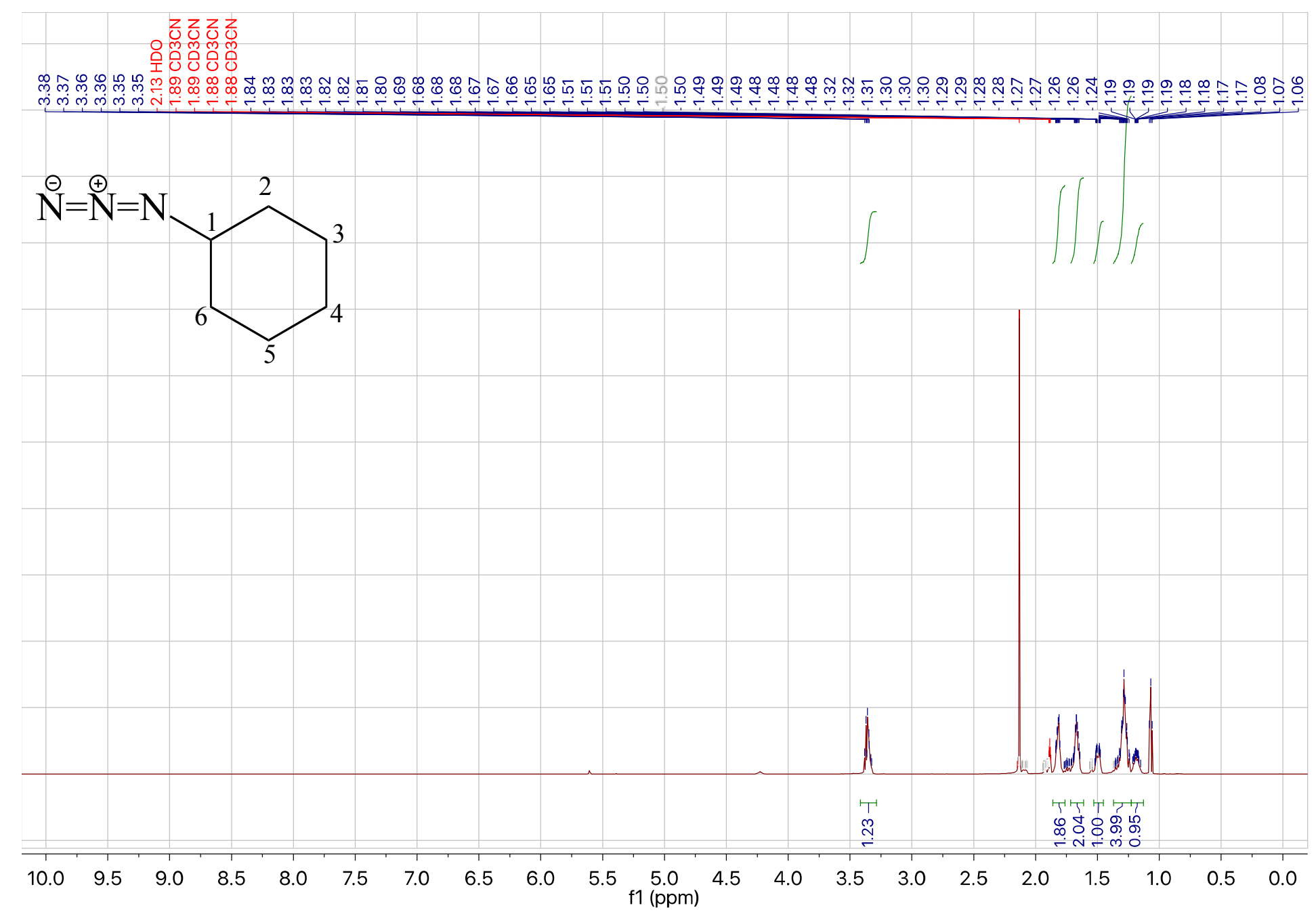

Figure 41S. ${ }^{1} \mathrm{H}$ NMR spectrum of compound $\mathbf{A Z 2}$ in $\mathrm{CD}_{3} \mathrm{CN}$. 


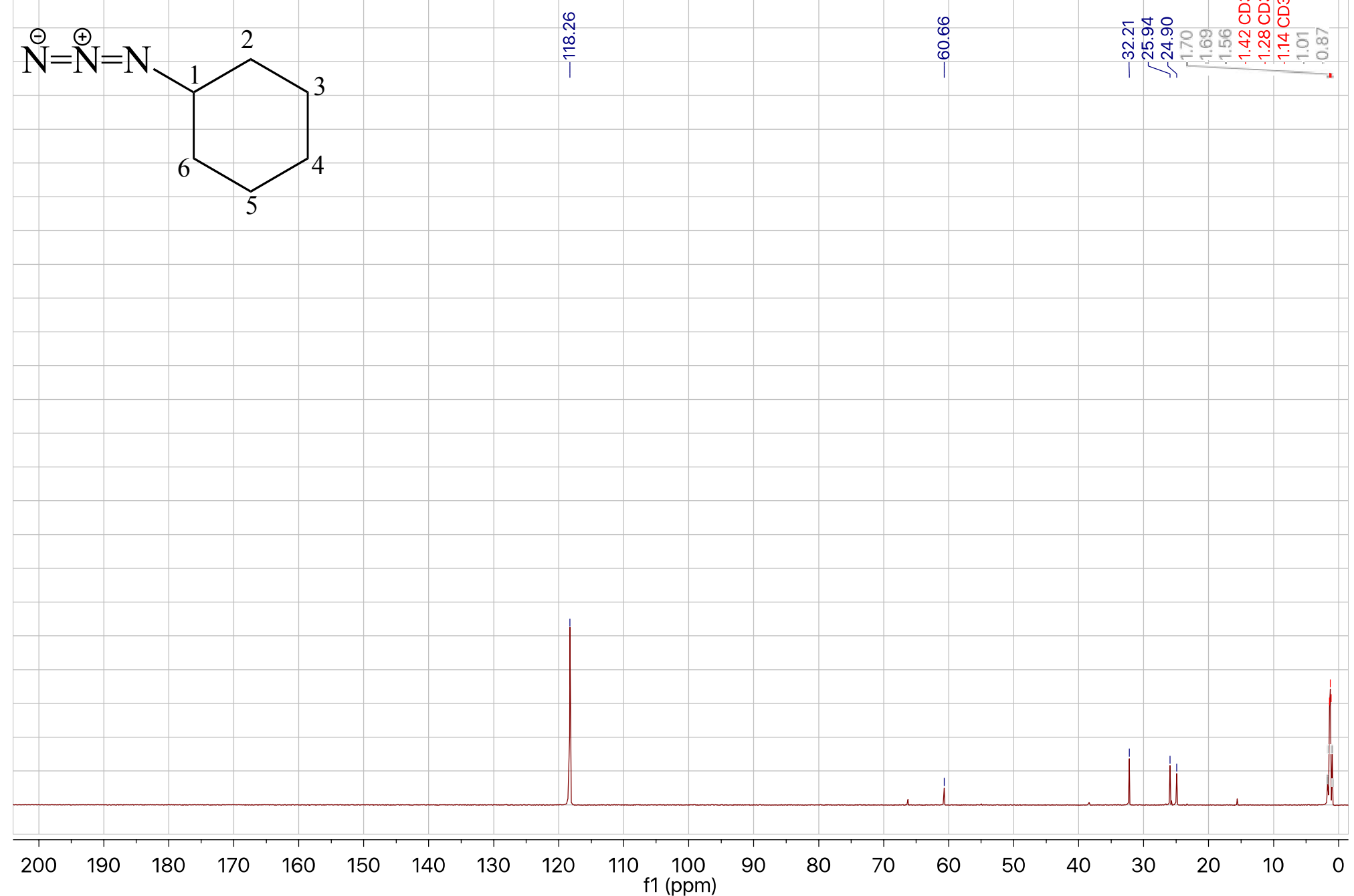

Figure 42S. ${ }^{13} \mathrm{C}$ NMR spectrum of compound $\mathbf{A Z 2}$ in $\mathrm{CD}_{3} \mathrm{CN}$. 


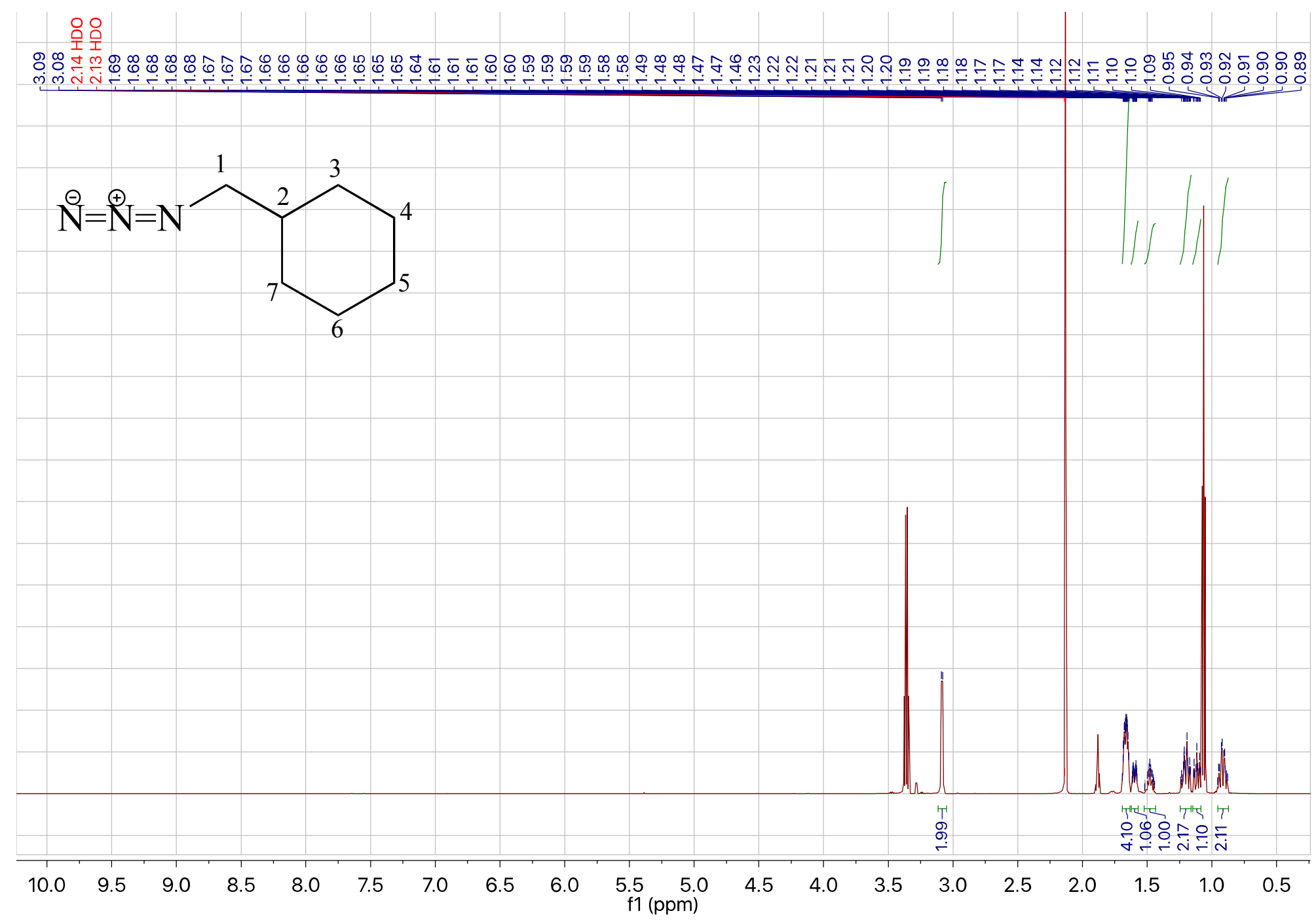

Figure 43S. ${ }^{1} \mathrm{H}$ NMR spectrum of compound $\mathbf{A Z 3}$ in $\mathrm{CD}_{3} \mathrm{CN}$. 


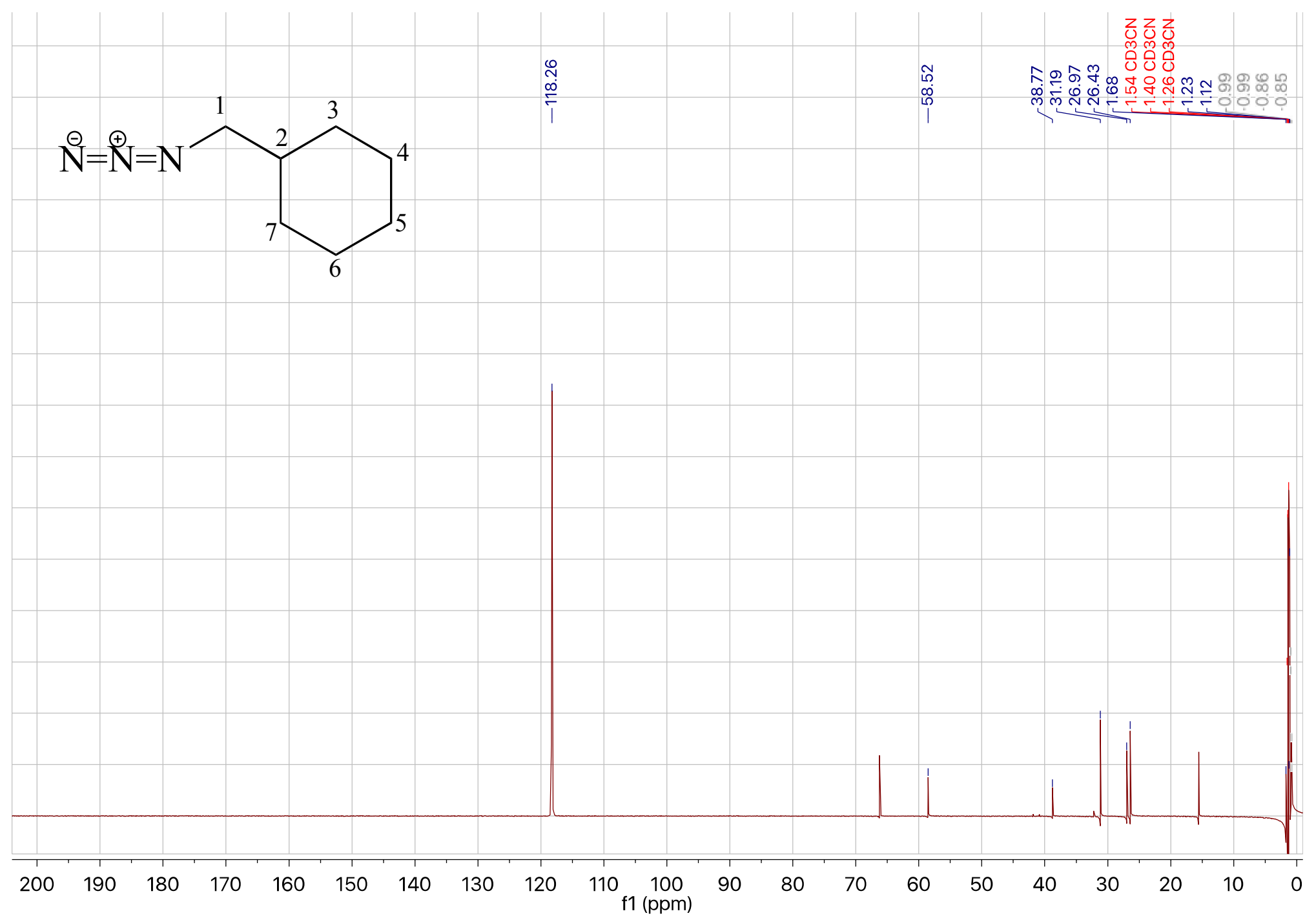

Figure 44S. ${ }^{13} \mathrm{C}$ NMR spectrum of compound $\mathbf{A Z 3}$ in $\mathrm{CD}_{3} \mathrm{CN}$. 


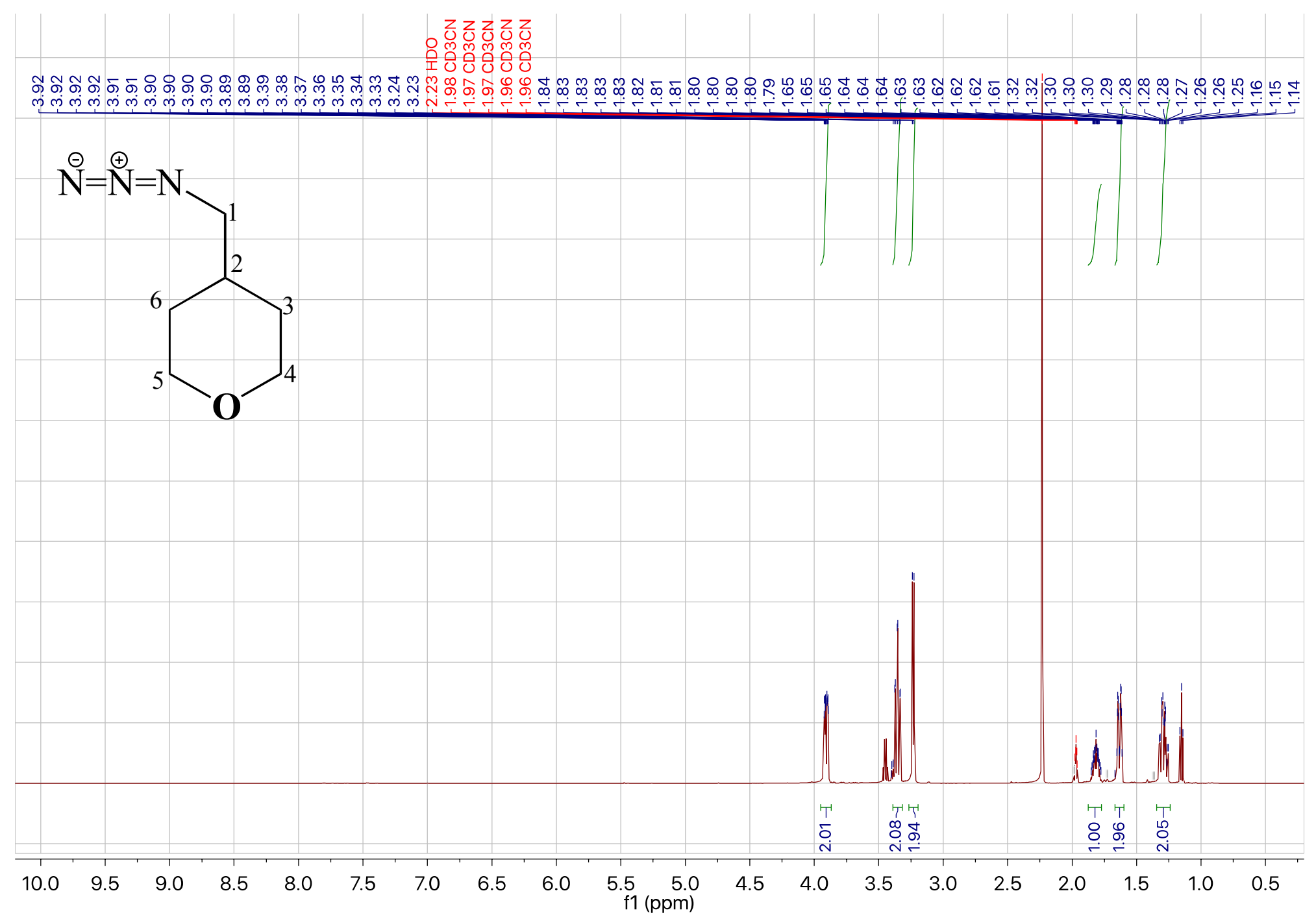

Figure 45S. ${ }^{1} \mathrm{H}$ NMR spectrum of compound $\mathbf{A Z 4}$ in $\mathrm{CD}_{3} \mathrm{CN}$. 


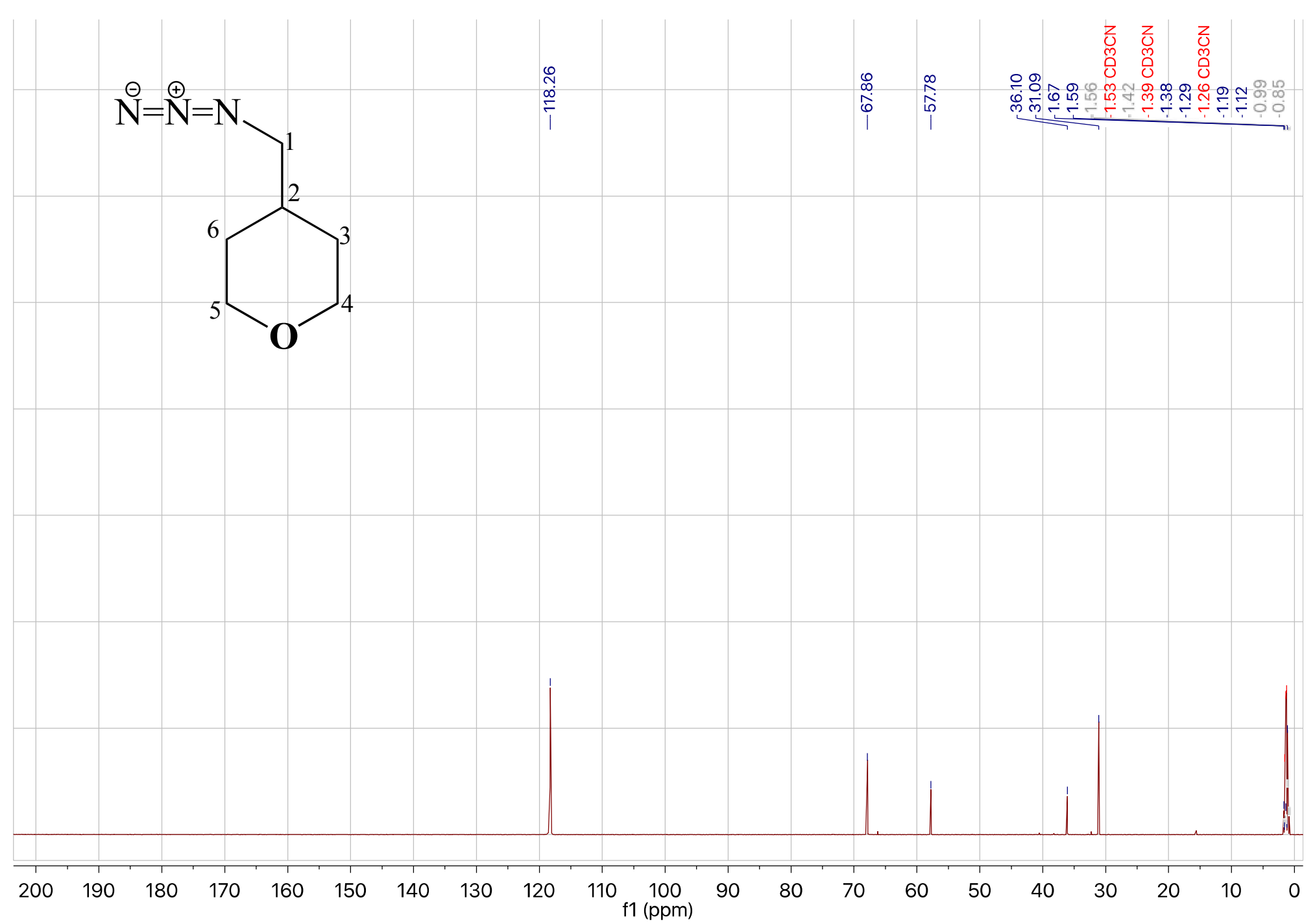

Figure 46S. ${ }^{13} \mathrm{C}$ NMR spectrum of compound $\mathbf{A Z 4}$ in $\mathrm{CD}_{3} \mathrm{CN}$. 


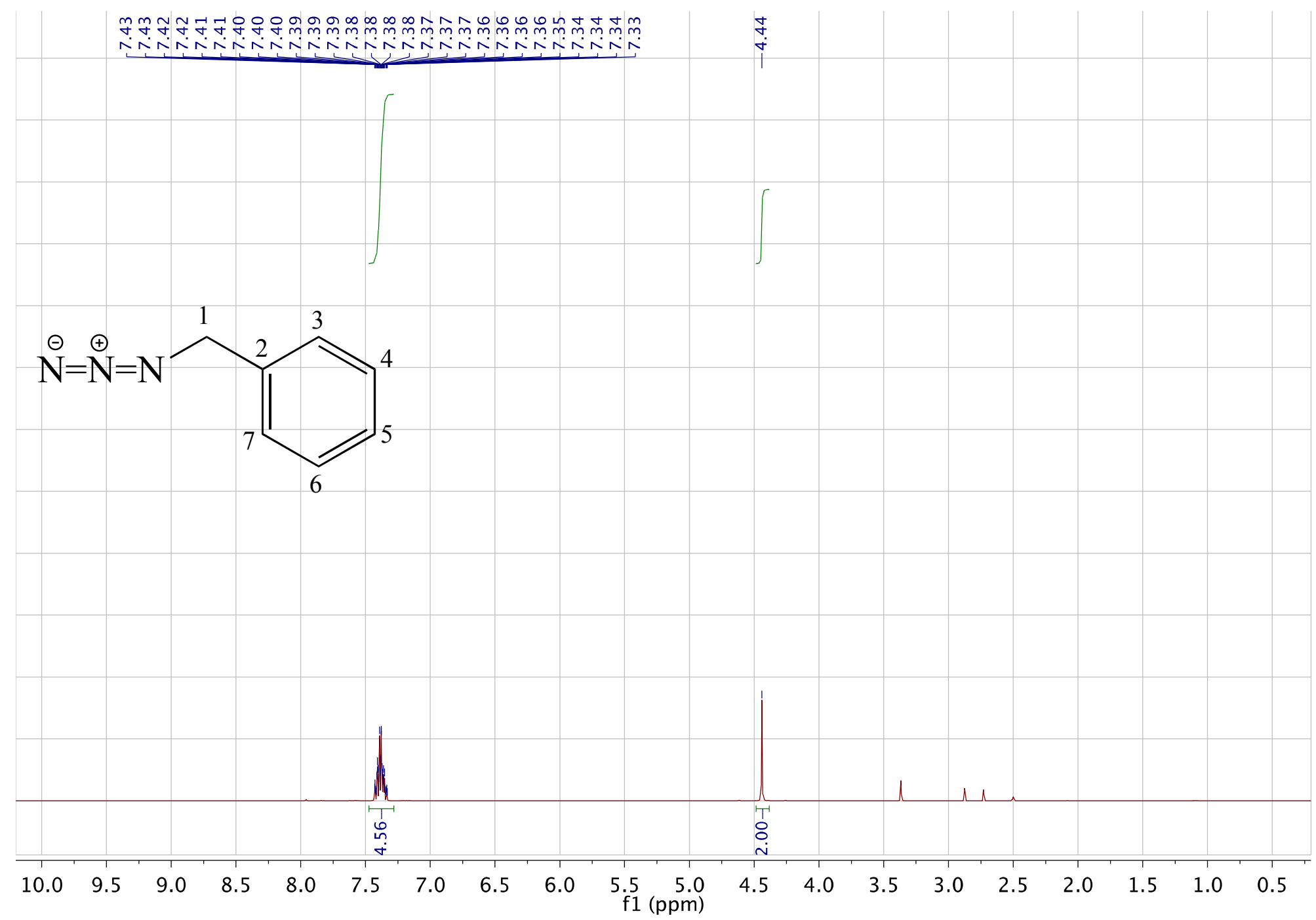

Figure 47S. ${ }^{1} \mathrm{H}$ NMR spectrum of compound AZ5 in DMSO-d $\mathrm{d}_{6}$. 


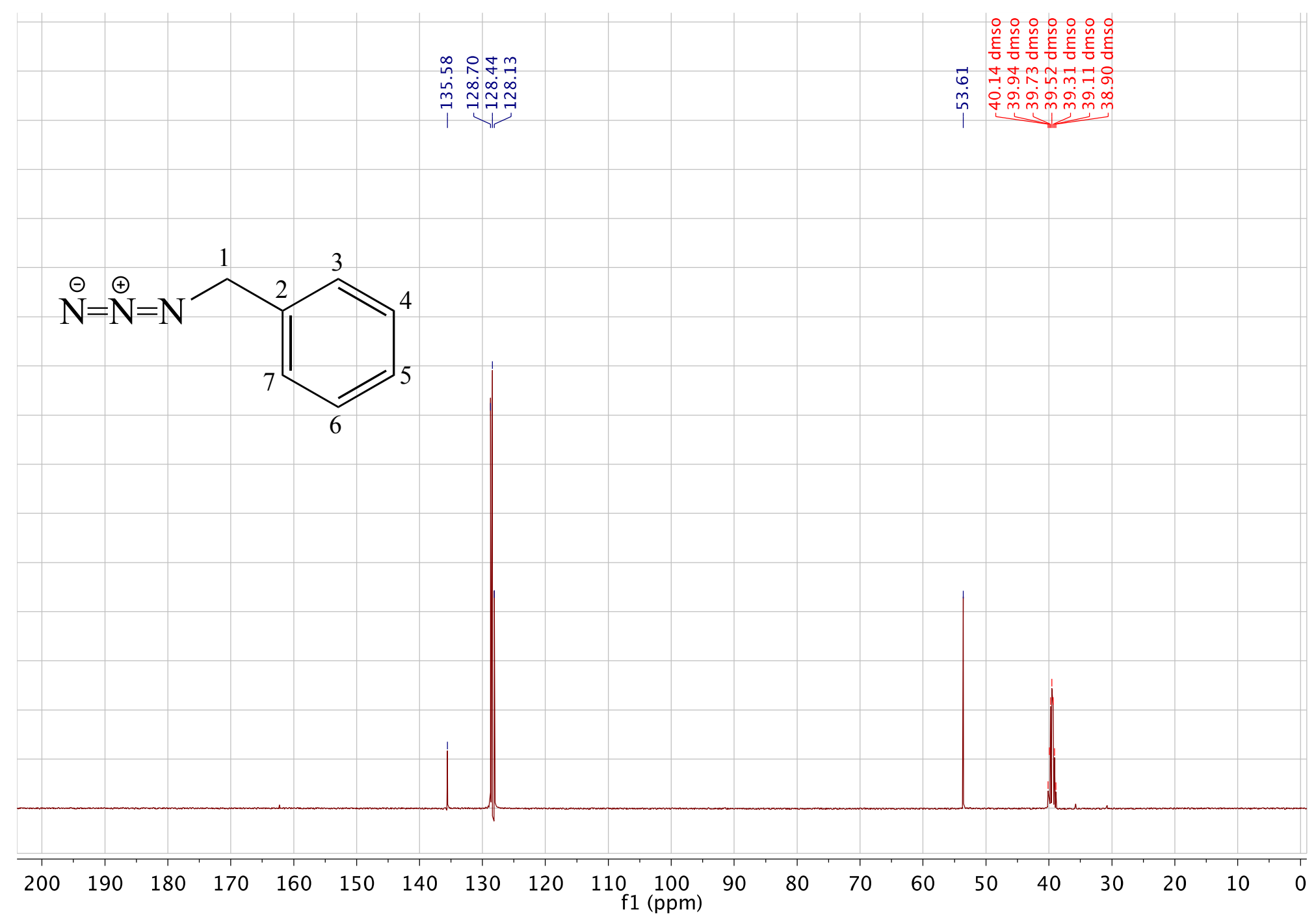

Figure $48 \mathrm{~S} .{ }^{13} \mathrm{C}$ NMR spectrum of compound AZ5 in DMSO-d $\mathrm{d}_{6}$. 
(a)

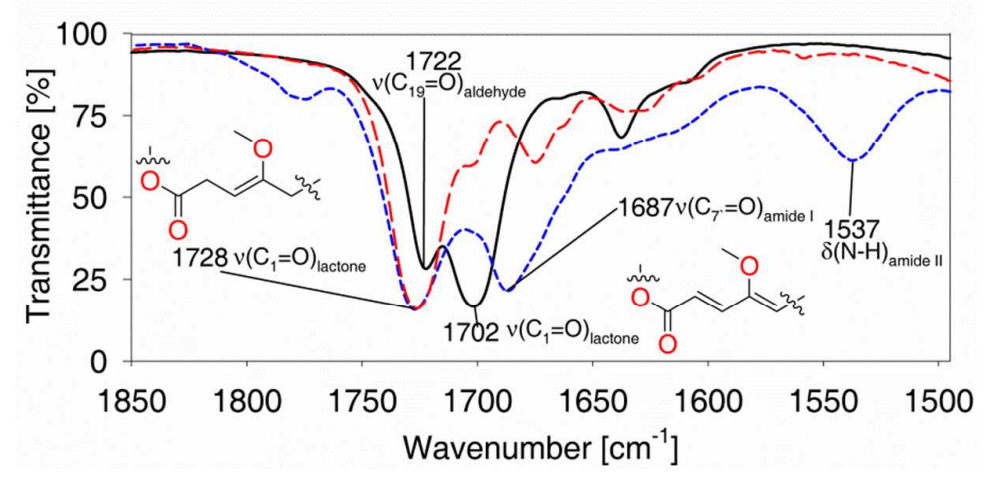

(b)

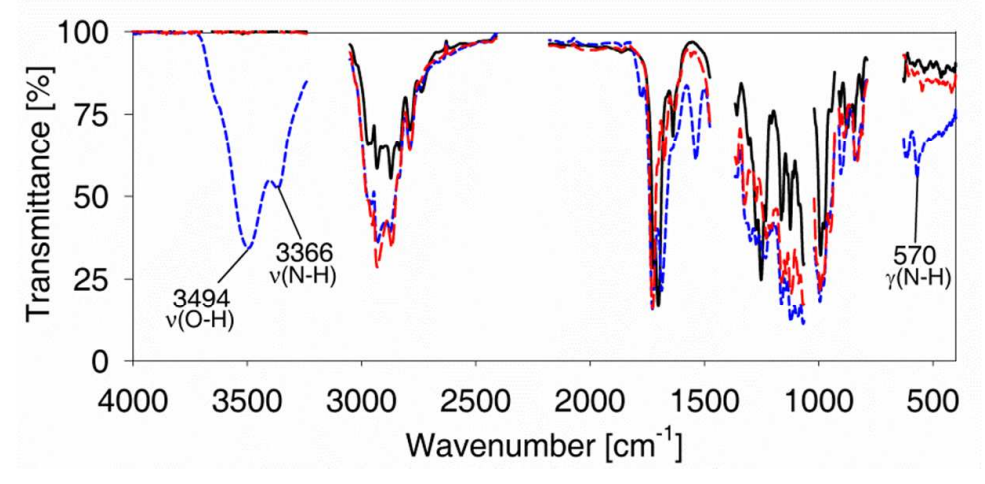

Figure 49S. Comparison of FT-IR spectra in: (a) 1850-1500 $\mathrm{cm}^{-1}$ and (b) $4000-450 \mathrm{~cm}^{-1}$ ranges between 2 (black solid) and those of novel triazole conjugates: 8 (blue short-dashed) and $\mathbf{1 1}$ (red long dashed); all spectra recorded in $\mathrm{CH}_{3} \mathrm{CN}(c 0.05 \mathrm{M})$. 


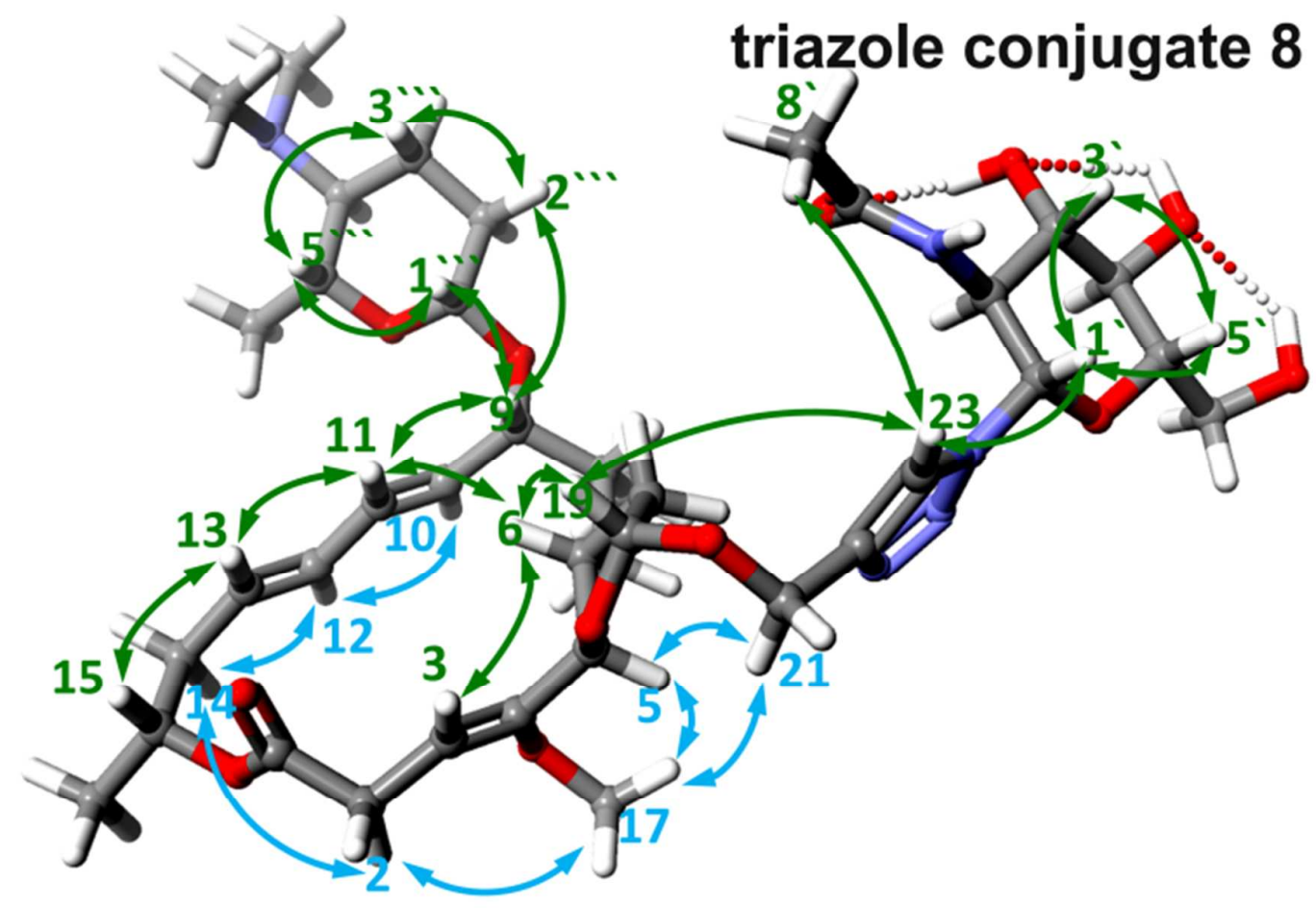

Figure 50S. Key ${ }^{1} \mathrm{H}-{ }^{1} \mathrm{H}$ NOESY contacts recorded for compound $\mathbf{8}$ showing the mutual arrangement of the reconstructed $\mathrm{C}(5)$ arm and the aglycone part of antibiotic, shown on the lowest-energy structure $\left(\mathrm{H}_{\mathrm{f}}^{\circ}=-539.27 \mathrm{kcal} / \mathrm{mol}\right)$ calculated by B88-LYP (GGA) DFT method /Scigress Explorer package FJ 2.4. EU 3.1.8/. 

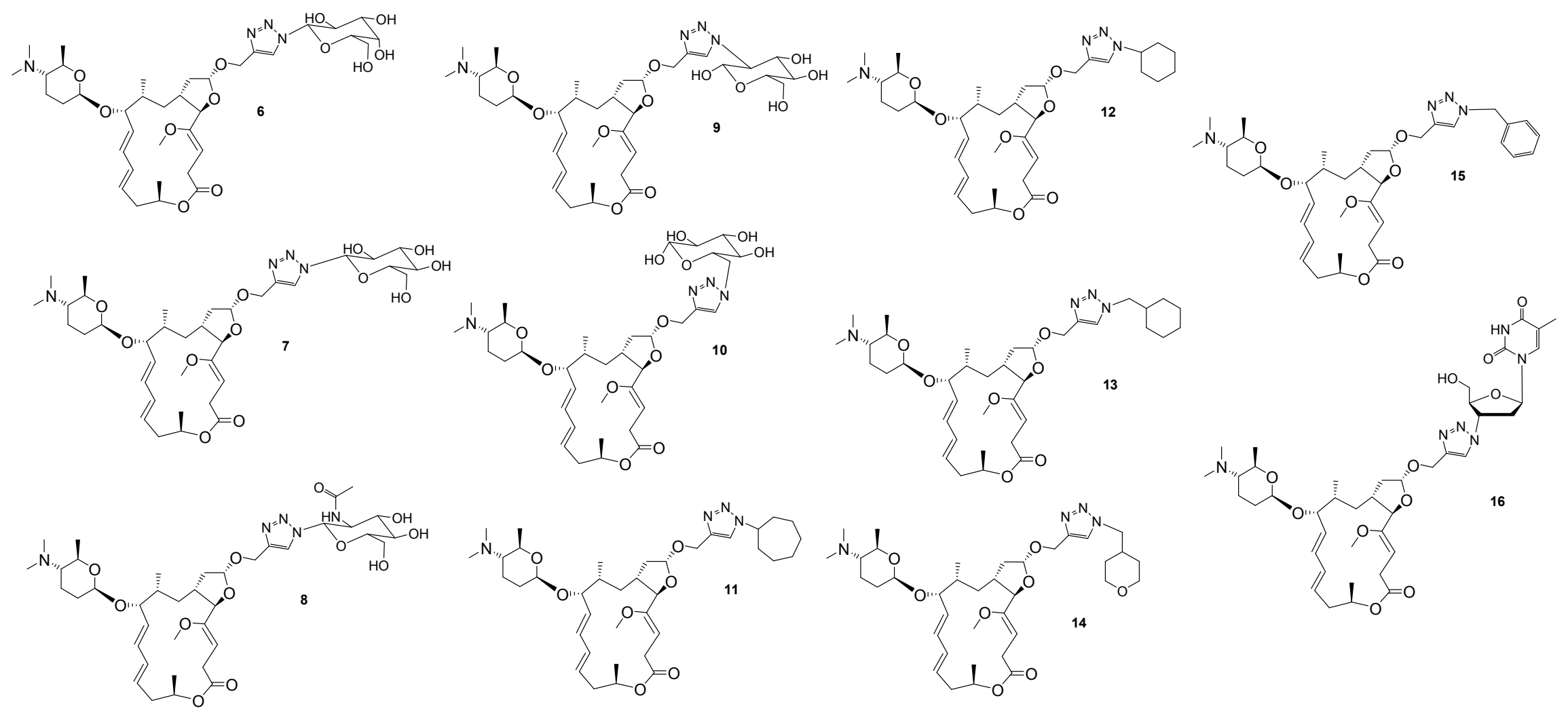

Figure 51S. Structures of all novel chemical entities 6-16 presented in ChemDraw 8.0 format 